\title{
Discrete Morse Theory and Classifying Spaces
}

\author{
Vidit Nanda, Dai Tamaki, and Kohei Tanaka
}

\begin{abstract}
The aim of this paper is to develop a refinement of Forman's discrete Morse theory. To an acyclic partial matching $\mu$ on a finite regular CW complex $X$, Forman introduced a discrete analogue of gradient flows. Although Forman's gradient flow has been proved to be useful in practical computations of homology groups, it is not sufficient to recover the homotopy type of $X$. Forman also proved the existence of a CW complex which is homotopy equivalent to $X$ and whose cells are in one-to-one correspondence with the critical cells of $\mu$, but the construction is ad hoc and does not have a combinatorial description. By relaxing the definition of Forman's gradient flows, we introduce the notion of flow paths, which contains enough information to reconstruct the homotopy type of $X$, while retaining a combinatorial description. The critical difference from Forman's gradient flows is the existence of a partial order on the set of flow paths, from which a 2-category $C(\mu)$ is constructed. It is shown that the classifying space of $C(\mu)$ is homotopy equivalent to $X$ by using homotopy theory of 2-categories. This result can be also regarded as a discrete analogue of the unpublished work of Cohen, Jones, and Segal on Morse theory in early 90's.
\end{abstract}

\section{Contents}

1 Introduction

1.1 Discrete Morse Theory . . . . . . . . . . . . . . . . . . . . . . . 2

1.2 Main Result . . . . . . . . . . . . . . . . . . . . . . . . 3

1.3 Organization of the Paper . . . . . . . . . . . . . . . . . 4

1.4 Acknowledgments . . . . . . . . . . . . . . . . . . . . 5

2 Discrete Morse Theory

2.1 Discrete Morse Functions . . . . . . . . . . . . . . . . . . 5

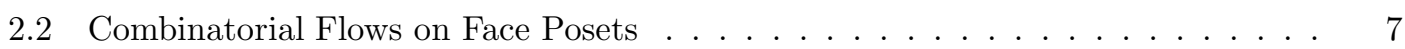

2.3 From Combinatorial Flows to Stable Subdivision . . . . . . . . . . . . . . . . 13

3 The Flow Category 21

3.1 Small Categories . . . . . . . . . . . . . . . . . . . . . . 22

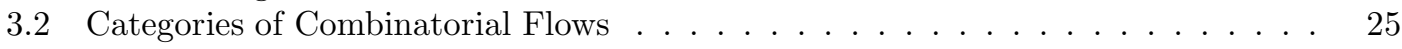

3.3 The Collapsing Functor . . . . . . . . . . . . . . . . . . . . . 31

3.4 The Face Poset of Stable Subdivision . . . . . . . . . . . . . . . . . . . . . 37

A Homotopy Theory of Small Categories $4 \ldots$

A.1 Simplicial Sets and Simplicial Spaces . . . . . . . . . . . . . . . . . . . . . . . . 42

A.2 Nerves and Classifying Spaces . . . . . . . . . . . . . . . . . . . . 44

A.3 Comma Categories and Quillen's Theorem A . . . . . . . . . . . . . . . . 48

A.4 Comma Categories and Quillen's Theorem A for 2-Categories . . . . . . . . . . 49 


\section{Introduction}

The goal of this paper is to describe a new combinatorial theory of gradient flow on cell complexes which provides direct insight into discrete Morse homotopy, and hence to extend Forman's discrete Morse theory [For95, For98].

\subsection{Discrete Morse Theory}

The central objects of study in Forman's adaptation of Morse theory to CW complexes are discrete Morse functions, which assign (real) values to cells. Every discrete Morse function $f$ on a regular CW complex $X$ imposes a partial pairing on its constituent cells, i.e. a bijection $\mu_{f}: D\left(\mu_{f}\right) \rightarrow U\left(\mu_{f}\right)$ between disjoint subsets $D\left(\mu_{f}\right), U\left(\mu_{f}\right)$ of the face poset $F(X)$. The unpaired cells are analogous to critical points from smooth Morse theory, while the paired cells generate combinatorial gradient paths between critical ones. Such gradient paths have been proved to be quite useful in computing homology and cohomology in various contexts such as topological combinatorics [BBL ${ }^{+} 99$, Sha01], hyperplane arrangements [SS07, Del08, DS10], cohomology of algebraic structures [BW02, Koz05, Skö06, JW09, BBM07], and topological data analysis [MN13].

Compared to these nice applications to homology, it is disappointing to see the failure of the use of Forman's gradient paths to the study of homotopy types. Recall that a gradient path, in the sense of Forman, from a critical cell $c$ to another $c^{\prime}$ is an alternating sequence of cells

$$
c \succ d_{1} \prec u_{1} \succ \cdots \prec u_{k-1} \succ d_{k} \prec u_{k} \succ \cdots \prec u_{n} \succ c^{\prime},
$$

where $d_{i} \in D\left(\mu_{f}\right)$ and $u_{i} \in U\left(\mu_{f}\right)$ with $\mu_{f}\left(d_{i}\right)=u_{i}$, and all the face relations $\prec$ and $\succ$ appearing in this sequence are of codimension 1 , which imposes the dimension constraint $\operatorname{dim} c=\operatorname{dim} c^{\prime}+1$. This failure is best illustrated by the following simple example. Consider the "height function" $h$ on the boundary of a 3 -simplex $\Delta^{3}=\left[v_{0}, v_{1}, v_{2}, v_{3}\right]$, whose associated partial matching $\mu_{h}$ is indicated by arrows in Figure 1. For example, the vertex $\left[v_{1}\right]$ is matched with the edge $\left[v_{0}, v_{1}\right]$

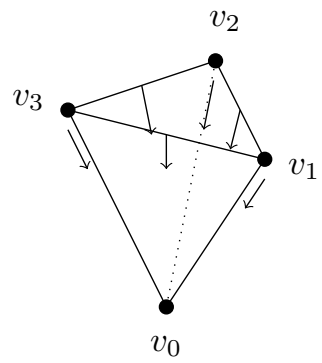

Figure 1: A partial matching on $\partial \Delta^{3}$

and the edge $\left[v_{1}, v_{2}\right]$ is matched with the face $\left[v_{0}, v_{1}, v_{2}\right]$. The remaining two cells, the bottom vertex $\left[v_{0}\right]$ and the top face $\left[v_{1}, v_{2}, v_{3}\right]$, are critical. There is no gradient path between these two critical cells because of the dimension gap, although there is an apparent deformation indicated by the arrows, which results in the minimal cell decomposition of a sphere $S^{2}=e^{0} \cup e^{2}$.

In fact, Forman generalized the deformation in the previous example and showed that matched pairs of cells can be collapsed together without changing the homotopy type. Thus we obtain a new cell complex $X_{f}$, whose cells are indexed by the set $\operatorname{Cr}(f)$ of critical cells of $f$. However, 
there is no explicit description of the resulting cell complex in terms of gradient paths. In smooth Morse theory, on the other hand, there is a well-known construction of a cell decomposition of a smooth manifold $M$ from gradient flows generated by a Morse-Smale function $f: M \rightarrow \mathbb{R}$, whose cells are indexed by critical points [Kal75, Fra79].

\subsection{Main Result}

In this paper, we introduce flow paths of acyclic partial matchings. These generalize Forman's gradient paths, and they may be used to explicitly recover the homotopy type of the original cell complex.

Definition 1.1. A flow path from a critical cell $c$ to another $c^{\prime}$ is a sequence of cells of the following form

$$
\begin{aligned}
c \succ u_{1} \succ \cdots \succ u_{i_{1}-1} \succ d_{i_{1}} \prec \mu_{f}\left(d_{i_{1}}\right)=u_{i_{1}} & \succ \cdots \\
& \succ u_{i_{2}-1} \succ d_{i_{2}} \prec \mu_{f}\left(d_{i_{2}}\right)=u_{i_{2}} \succ \cdots \succ u_{n} \succ c^{\prime}
\end{aligned}
$$

or

$$
\begin{aligned}
c \succ d_{1} \prec \mu_{f}\left(d_{1}\right)=u_{1} \succ \cdots \succ u_{i_{1}-1} \succ d_{i_{1}} & \prec \mu_{f}\left(d_{i_{1}}\right)=u_{i_{1}} \succ \cdots \\
& \succ u_{i_{2}-1} \succ d_{i_{2}} \prec \mu_{f}\left(d_{i_{2}}\right)=u_{i_{2}} \succ \cdots \succ u_{n} \succ c^{\prime},
\end{aligned}
$$

where $u_{i} \in U\left(\mu_{f}\right)$ and $d_{i} \in D\left(\mu_{f}\right)$.

There are two differences from Forman's gradient paths. We allow descending sequences of cells in $U\left(\mu_{f}\right)$ to appear in a flow path and the face relations $u_{i_{k}-1} \succ d_{i_{k}}$ have no codimension restrictions.

This simple extension of gradient paths turns out to contain enough information to reconstruct the homotopy type. The crucial property is the existence of a partial order on the $\operatorname{set} \operatorname{FP}\left(\mu_{f}\right)$ of all flow paths, which induces a partial order on the set $C\left(\mu_{f}\right)\left(c, c^{\prime}\right)$ of flow paths from $c^{\prime}$ to $c$.

Recall that there is a standard way of constructing a simplicial complex $B P$ from a poset $P$, called the order complex [Bjö95]. Regarding posets as a special class of small categories, the order complex construction has been extended to small categories as the classifying space construction [Seg68]. By regarding the poset $C\left(\mu_{f}\right)\left(c, c^{\prime}\right)$ as a small category, our flow category $C\left(\mu_{f}\right)$ becomes a category enriched over the category of small categories, i.e. a (strict) 2-category [Lac10]. The classifying space construction has been also extended to 2-categories, with which our main theorem can be stated as follows.

Theorem 1.2. Let $X$ be a finite regular $C W$ complex and $f$ a discrete Morse function on the face poset $F(X)$. Then the classifying space of the flow category $C\left(\mu_{f}\right)$ is homotopy equivalent to $X$.

Note that there are several ways to take classifying spaces of 2-categories. One of the most popular models is the classifying space of the topological category obtained by taking the classifying space of each morphism category. This construction is denoted by $B^{2}$ in this paper. We also make use of the "normal colax version" $B^{n c l}$ in the proof of the theorem. It is known that these two, including several other constructions, are homotopy equivalent to each other. This is proved by Carrasco, Cegarra, and Garzón in [CCG10].

The classifying space $B^{2} C\left(\mu_{f}\right)$ is described by the combinatorial data associated to a discrete Morse function or an acyclic partial matching. Thus it provides a systematic way of reconstructing the homotopy type of the original cell complex purely in terms of Morse data. 
For example, this description as the classifying space of a 2-category or a topological category can serve as an alternative systematic approach to computing homology via discrete Morse theory. Given a multiplicative homology theory $h_{*}(-)$ satisfying the strong form of the Künneth isomorphism, the homotopy equivalence in Theorem 1.2 gives rise to a spectral sequence

$$
E^{2} \cong H_{*}\left(h_{*}\left(B C\left(\mu_{f}\right)\right)\right) \Longrightarrow h_{*}\left(B^{2} C\left(\mu_{f}\right)\right) \cong h_{*}(X)
$$

by [Seg68], where $h_{*}\left(B C\left(\mu_{f}\right)\right)$ is the category enriched over graded $h_{*}$-modules whose set of objects is $\operatorname{Cr}\left(\mu_{f}\right)$ and whose module of morphisms from $c$ to $c^{\prime}$ is $h_{*}\left(B C\left(\mu_{f}\right)\left(c, c^{\prime}\right)\right)$. And $H_{*}(-)$ is the homology of linear categories, studied, for example, in [Wat66].

As a version of Morse theory, it would be desirable to find a cell decomposition of $B^{2} C\left(\mu_{f}\right)$ or $B^{n c l} C\left(\mu_{f}\right)$ indexed by $\operatorname{Cr}\left(\mu_{f}\right)$. Our categorical construction is also useful to study this problem. We discuss this problem in a separate paper.

One of the sources of inspiration for this work is an unpublished manuscript of Ralph Cohen, John Jones, and Graeme Segal [CJS] that appeared in early 90's, in which they proposed a way to reconstruct the homotopy type (and even a homeomorphism type) of a smooth manifold from a topological category consisting of critical points and moduli spaces of gradient flows associated to a Morse-Smale function. Our main theorem can be regarded as a realization of their proposal in a discrete setting. Another possible use of our result is, therefore, to give an alternative proof of the homotopy-equivalence part of Cohen-Jones-Segal Morse theory and its extensions. As is done by Gallais [Gal10], it is possible to use discrete Morse theory to approximate smooth Morse theory. The original motivation of the paper [CJS] seems to develop a toy model for Floer homotopy theory [CJS94, CJS95]. It would be interesting if the theory of flow paths developed in this paper provides an alternative approach to Floer homotopy theory.

\subsection{Organization of the Paper}

The paper is organized as follows.

- We recall basics of discrete Morse theory in $\S 2.1$ to fix notation and terminology. The notion of flow paths is introduced in $\S 2.2$ together with a partial order on the set $\operatorname{FP}(\mu)$ of all flow paths with respect to a partial matching $\mu$. We also introduce reduced flow paths and compare the set $\overline{\mathrm{FP}}(\mu)$ of reduced flow paths with $\operatorname{FP}(\mu)$. In $\S 2.3$, we introduce geometric flows associated to flow paths to define a subdivision $\operatorname{Sd}_{\mu}(X)$ of $X$.

- $\S 3$ is the main body of this paper. After reviewing notation and terminology for small categories in $\S 3.1$, the flow category $C(\mu)$ and its reduced version $\bar{C}(\mu)$ are introduced in $\S 3.2$.

Theorem 1.2 is proved as follows

1. In the first half of $\S 3.3$, we construct a normal colax functor $\tau: \operatorname{FP}(\mu) \rightarrow C(\mu)$, called the collapsing functor, by assigning the terminal cell to each flow path. It is shown that the collapsing functor can be restricted to a functor $\tau: \overline{\mathrm{FP}}(\mu) \longrightarrow \bar{C}(\mu)$.

2 . In the second half of $\S 3.3$, we show that $\tau$ induces a homotopy equivalence between "normal colax" classifying spaces

$$
B^{n c l} \tau: B \overline{\mathrm{FP}}(\mu)=B^{n c l} \overline{\mathrm{FP}}(\mu) \stackrel{\simeq}{\longrightarrow} B^{n c l} \bar{C}(\mu)
$$

by a 2-categorical version of Quillen's Theorem A. 
3. In $\S 3.4$, we show that the face poset of the subdivision $\operatorname{Sd}_{\mu}(X)$ constructed in $\S 2.3$ is isomorphic to $\overline{\mathrm{FP}}(\mu)$. Thus we obtain a chain of homotopy equivalences

$$
X \cong B F\left(\operatorname{Sd}_{\mu}(X)\right) \cong B \overline{\mathrm{FP}}(\mu) \simeq B^{n c l} \bar{C}(\mu) \simeq B^{2} \bar{C}(\mu) \simeq B^{2} C(\mu) .
$$

- An appendix on one of our main tools, i.e. homotopy theory of small categories, is attached at the end of this paper with the hope of making this article self-contained.

Although homotopy theory of small categories has been an indispensable tool in topology and combinatorics since Segal [Seg68] and Quillen [Qui73], there seems to be no standard reference for novices.

\subsection{Acknowledgments}

This work was initiated by VN when he met DT during the workshop in Hakata, Japan on applied topology organized by Yasu Hiraoka in 2011. We would like to thank Yasu for organizing the meeting. VN and DT would also like to thank Rob Ghrist for his encouragement during the workshop and other occasions, without which this work would not have been completed. Finally DT appreciates the kindness of Martin Guest who handed him a copy of Cohen-Jones-Segal paper [CJS] when DT was a graduate student at the University of Rochester in early 90's.

DT is supported by JSPS KAKENHI Grant Number JP23540082 and JP15K04870. KT is supported by JSPS KAKENHI Grant Number JP15K17535.

\section{Discrete Morse Theory}

The main aim of this section is to introduce the notion of flow paths for discrete Morse functions.

\subsection{Discrete Morse Functions}

Let us briefly recall Forman's discrete Morse theory. Throughout the rest of this section, we fix a regular CW complex $X$, whose face poset is denoted by $F(X)$. We denote the partial order in $F(X)$ by $e \preceq e^{\prime}$, which means that $e$ is a face of $e^{\prime}$. Moreover, we use $\prec_{1}$ to denote the cover relation, i.e. $e \prec_{1} e^{\prime}$ if and only if $e \preceq e^{\prime}$ and $\operatorname{dim} e=\operatorname{dim} e^{\prime}-1$.

Definition 2.1. For a function $f: F(X) \rightarrow \mathbb{R}$ and a cell $e \in F(X)$, define

$$
\begin{aligned}
& N_{f}^{+}(e)=\left\{e^{\prime} \in F(X) \mid e \prec_{1} e^{\prime}, f(e) \geq f\left(e^{\prime}\right)\right\} \\
& N_{f}^{-}(e)=\left\{e^{\prime} \in F(X) \mid e^{\prime} \prec_{1} e, f\left(e^{\prime}\right) \geq f(e)\right\} .
\end{aligned}
$$

$f$ is called a discrete Morse function if $\left|N_{f}^{+}(e)\right| \leq 1$ and $\left|N_{f}^{-}(e)\right| \leq 1$ for all $e \in F(X)$. A cell $e \in F(X)$ is said to be critical if $N_{f}^{+}(e)=N_{f}^{-}(e)=\emptyset$. The set of critical cells is denoted by $\operatorname{Cr}(f)$.

Remark 2.2. We sometimes regard $f$ as a locally constant function on $X$ under the composition $X \stackrel{\pi_{X}}{\longrightarrow} F(X) \stackrel{f}{\longrightarrow} \mathbb{R}$, where $\pi_{X}$ is the map which defines the cell decomposition or stratification of $X$, i.e. $\pi_{X}(x)=e$ if $x \in e$.

Forman observed that, for a noncritical cell $e$, either $\left|N_{f}^{+}(e)\right|=1$ or $\left|N_{f}^{-}(e)\right|=1$ happens. This allows us to define a partial matching on the face poset $F(X)$. 
Definition 2.3. A partial matching on the face poset $F(X)$ is a bijection $\mu: D(\mu) \rightarrow U(\mu)$ between disjoint subsets $D(\mu), U(\mu)$ of $F(X)$ such that $d \prec_{1} \mu(d)$ for each $d \in D(\mu)$. Elements of

$$
\operatorname{Cr}(\mu)=F(X) \backslash D(\mu) \cup U(\mu)
$$

are called critical.

Definition 2.4. Given a discrete Morse function $f$ on $F(X)$, define a partial matching $\mu_{f}$ as follows. The domain and the range of $\mu_{f}$ are given by

$$
\begin{aligned}
D\left(\mu_{f}\right) & =\left\{e \in F(X) \mid N_{f}^{+}(e) \neq \emptyset\right\} \\
U\left(\mu_{f}\right) & =\left\{e \in F(X) \mid N_{f}^{-}(e) \neq \emptyset\right\} .
\end{aligned}
$$

For $e \in D\left(\mu_{f}\right), \mu_{f}(e)$ is the unique cell in $N_{f}^{+}(e)$.

Note that $\operatorname{Cr}(f)=\operatorname{Cr}\left(\mu_{f}\right)$ for a discrete Morse function $f$. We identify two discrete Morse functions when their partial matchings agree.

Definition 2.5. Two discrete Morse functions $f$ and $g$ on a regular CW complex $X$ are said to be equivalent if, for every pair $e \prec_{1} e^{\prime}$ of cells in $X$, we have

$$
f(e)<f\left(e^{\prime}\right) \Longleftrightarrow g(e)<g\left(e^{\prime}\right) .
$$

Definition 2.6. A Forman path $\rho$ with respect to a partial matching $\mu$ is a sequence of distinct noncritical cells

$$
\rho: d_{1} \prec \mu\left(d_{1}\right) \succ d_{2} \prec \mu\left(d_{2}\right) \succ \cdots \succ d_{n} \prec \mu\left(d_{n}\right),
$$

with $\operatorname{dim} d_{i+1}=\operatorname{dim} \mu\left(d_{i}\right)-1$. Equivalently it is a sequence $\rho=\left(d_{1}, \ldots, d_{n}\right)$ of distinct cells of the same dimension in $D(\mu)$ with $\mu\left(d_{i}\right) \succ d_{i+1}$ for all $i=1, \ldots, n-1$. $\rho$ is called a gradient path if either $n=1$ or $d_{1} \nprec \mu\left(d_{n}\right)$.

Forman observed that partial matchings associated to discrete Morse functions have the following significant property.

Definition 2.7. A partial matching is called acyclic if all of its Forman paths are gradient.

Conversely, it is known that any acyclic partial matching comes from a discrete Morse function.

Proposition 2.8. For any acyclic partial matching $\mu$ on a finite regular $C W$ complex $X$, there exists a discrete Morse function $f$ on $X$ with $\mu=\mu_{f}$.

Proof. See Theorem 9.3 in [For95].

This implies that discrete Morse theory can be developed entirely in terms of acyclic partial matchings. It is, however, useful to have good discrete Morse functions at hand.

Forman already observed that any discrete Morse function on a finite CW complex is equivalent to an injective one ${ }^{1}$, which can be perturbed further into a faithful Morse function.

Definition 2.9. A discrete Morse function $f$ is said to be faithful if it satisfies the following conditions:

\footnotetext{
${ }^{1}$ See the first paragraph of the proof of Theorem 3.3 in [For98]
} 
1. $f$ is injective.

2. If $e \prec e^{\prime}$ and $e^{\prime} \neq \mu_{f}(e)$, then $f(e)<f\left(e^{\prime}\right)$.

Proposition 2.10. For any discrete Morse function $f$ on a finite regular $C W$ complex $X$, there exists a $\mathbb{Z}$-valued faithful discrete Morse function $\tilde{f}$ equivalent to $f$.

Our proof of this fact is closely related to the notion of flow paths, which will be introduced in §2.2. A proof of this proposition is given there, after Remark 2.12.

By Proposition 2.10, the discrete Morse function in Proposition 2.8 can be chosen to be faithful. In the rest of this paper, we fix an acyclic partial matching $\mu$ on a regular $\mathrm{CW}$ complex $X$. When $X$ is finite we also choose a faithful discrete Morse function $f$ with $\mu=\mu_{f}$. In particular, all critical values of $f$ are distinct.

\subsection{Combinatorial Flows on Face Posets}

As we have recalled in Definition 2.6, gradient paths in the sense of Forman can only connect pairs of cells of dimension difference 1. Such flows are enough for computations of homology. We need more general flows, called flow paths, to describe relations among cells of arbitrary dimension differences. We also need to define a partial order on the set of all flow paths to describe "the topology of the space of flow paths", which can be used to recover the homotopy type of the original CW complex.

Definition 2.11. A flow path with respect to an acyclic partial matching $\mu$ is a sequence $\gamma=\left(e_{1}, u_{1}, \ldots, e_{n}, u_{n} ; c\right)$ of cells satisfying the following conditions:

1. $u_{i} \in U(\mu)$ for $1 \leq i \leq n$;

2. either $e_{i}=u_{i}$ or $e_{i}=\mu^{-1}\left(u_{i}\right)$ for $1 \leq i \leq n$;

3. the last cell $c$ is critical;

4. $u_{i} \succ e_{i+1}$ for $1 \leq i \leq n$, where $e_{n+1}=c$;

The number $n$ is called the length of $\gamma$ and denoted by $\ell(\gamma)$. We allow $n$ to be 0 , in which case $\gamma=(c)$.

The critical cell $c$ is called the target of $\gamma$ and is denoted by $\tau(\gamma)$. The cell $e_{1}$ is called the initial cell of $\gamma$ and is denoted by $\iota(\gamma)$. When $\ell(\gamma)=0, \iota(\gamma)$ is defined to be $c$. We also use the notation $e_{n+1}=c$ when $n=\ell(\gamma)$. The set of flow paths with respect to $\mu$ is denoted by $\operatorname{FP}(\mu)$. When $\mu$ comes from a discrete Morse function $f$, it is also denoted by $\operatorname{FP}(f)$.

Remark 2.12. Recall that a Forman path is a sequence of cells of the following form

$$
d_{1} \prec \mu\left(d_{1}\right) \succ d_{2} \prec \mu\left(d_{2}\right) \succ \cdots \succ d_{n} \prec \mu\left(d_{n}\right) \succ c
$$

with $d_{i} \in D(\mu)$ or

$$
\mu^{-1}\left(u_{1}\right) \prec u_{1} \succ \mu^{-1}\left(u_{2}\right) \prec u_{2} \succ \cdots \succ \mu^{-1}\left(u_{n}\right) \prec u_{n} \succ c
$$

with $u_{i} \in U(\mu)$. And all the face relations are of codimension 1.

A flow path, on the other hand, is a sequence of the following form

$$
e_{1} \preceq u_{1} \succ e_{2} \preceq u_{2} \succ \cdots \succ e_{n} \preceq u_{n} \succ c
$$


in which either $e_{i}=u_{i}$ or $\mu^{-1}\left(u_{i}\right)$. And face relations $u_{i} \succ e_{i+1}$ are arbitrary.

Thus a flow path can be written as

$$
u_{1} \succ \cdots \succ u_{i_{1}-1} \succ \mu^{-1}\left(u_{i_{1}}\right) \prec u_{i_{1}} \succ \cdots \succ u_{i_{2}-1} \succ \mu^{-1}\left(u_{i_{2}}\right) \prec u_{i_{2}} \succ \cdots \succ u_{n} \succ c
$$

or

$$
\mu^{-1}\left(u_{1}\right) \prec u_{1} \succ \cdots \succ u_{i_{1}-1} \succ \mu^{-1}\left(u_{i_{1}}\right) \prec u_{i_{1}} \succ \cdots \succ u_{i_{2}-1} \succ \mu^{-1}\left(u_{i_{2}}\right) \prec u_{i_{2}} \succ \cdots \succ u_{n} \succ c
$$

depending on $e_{1}=u_{1}$ or $\mu^{-1}\left(u_{1}\right)$. Note that both of these sequences are considered to be of length $n$.

If $\mu=\mu_{f}$ for a faithful discrete Morse function $f$, a flow path $\gamma=\left(e_{1}, u_{1}, \ldots, e_{n}, u_{n} ; c\right)$ gives rise to a decreasing sequence of real numbers

$$
f\left(e_{1}\right) \geq f\left(u_{1}\right)>\cdots>f\left(e_{n}\right) \geq f\left(u_{n}\right)>f(\tau(\gamma)) .
$$

This is why we regard flow paths as a discrete analogue of gradient flows on smooth manifolds.

There is another interpretation of flow paths.

Definition 2.13. Define a relation $\triangleright_{1}$ on $F(X)$ as follows: $e \triangleright_{1} e^{\prime}$ if either $e \succeq e^{\prime}$ with $\mu\left(e^{\prime}\right) \neq e$, or $e \in D(\mu)$ and $\mu(e)=e^{\prime}$. Write $\unrhd$ for the transitive closure of $\triangleright_{1}$.

Remark 2.14. If $e \unrhd e^{\prime}$, there exists a sequence of cells

$$
e=e_{1} \triangleright_{1} e_{2} \triangleright_{1} \cdots \triangleright_{1} e_{n-1} \triangleright_{1} e_{n}=e^{\prime}
$$

Eliminating superfluous equalities, we see that such a sequence is either of the following forms

$$
e=e_{1} \succ \cdots \succ e_{i_{1}-1} \succ \mu^{-1}\left(e_{i_{1}}\right) \prec e_{i_{1}} \succ \cdots \succ e_{i_{2}-1} \succ \mu^{-1}\left(e_{i_{2}}\right) \prec e_{i_{2}} \succ \cdots \succ e_{n}=e^{\prime}
$$

or

$e=\mu^{-1}\left(e_{1}\right) \prec e_{2} \succ \cdots \succ e_{i_{1}-1} \succ \mu^{-1}\left(e_{i_{1}}\right) \prec e_{i_{1}} \succ \cdots \succ e_{i_{2}-1} \succ \mu^{-1}\left(e_{i_{2}}\right) \prec e_{i_{2}} \succ \cdots \succ e_{n}=e^{\prime}$, since there can be no successive sequence of two or more matched pairs $\mu^{-1}\left(e_{i}\right) \prec e_{i}$. In particular, $e \unrhd e^{\prime}$ and $\operatorname{dim} e=\operatorname{dim} e^{\prime}$ imply that the sequence is a strictly alternating sequence of $\prec_{1}$ and $\succ_{1}$ in which $\prec_{1}$ are matched pairs. In particular it is a Forman path in the sense of Definition 2.6.

It should be also noted that flow paths are special kind of such sequences, in which we require the last cell is critical and that cells $e_{i}$ belong to $U(\mu)$.

Lemma 2.15. The relation $\unrhd$ is a partial order on $F(X)$.

Proof. Since reflexivity and transitivity of $\unrhd$ follow immediately by definition, we establish antisymmetry. Assume, for contradiction, that $e \unrhd e^{\prime} \unrhd e$ holds for distinct cells $e$ and $e^{\prime}$ of $X$. Then, there must exit two chains $e \triangleright_{1} e_{1} \triangleright_{1} \cdots e_{m} \triangleright_{1} e^{\prime}$ and $e^{\prime} \triangleright_{1} e_{m+1} \triangleright_{1} \cdots \triangleright_{1} e_{m+n} \triangleright e$. By concatenating these flow paths, we obtain a sequence

$$
e \triangleright_{1} e_{1} \triangleright_{1} \cdots \triangleright_{1} e_{m} \triangleright_{1} e^{\prime} \triangleright_{1} e_{m+1} \triangleright_{1} \cdots \triangleright_{1} e_{m+n} \triangleright_{1} e
$$

By Remark 2.14, we obtain a Forman path from $e$ to itself, which contradicts the acyclicity of $\mu$.

We are now ready to prove Proposition 2.10. 
Proof of Proposition 2.10. By Lemma 2.15, the acyclic partial matching $\mu_{f}$ associated to $f$ defines a partial order $\unrhd$ on $F(X)$. Take a linear extension of this partial order and $g: F(X) \rightarrow \mathbb{N}$ be an injective enumeration of cells so that if $e \unrhd e^{\prime}$ then $g(e) \geq g\left(e^{\prime}\right)$. To see that $g$ is a discrete Morse function, suppose $e \prec_{1} e^{\prime}$ and $g(e) \geq g\left(e^{\prime}\right)$. By remark 2.14, this happens only when $e \in D\left(\mu_{f}\right)$ and $\mu_{f}(e)=e^{\prime}$. Thus, $g$ is a discrete Morse function with $\mu_{g}=\mu_{f}$. Since both $f$ and $g$ are injective, their equivalence follows immediately from the fact that their partial matchings coincide.

Remark 2.16. We may define a category whose objects are cells in $X$ and whose morphisms from $e$ to $e^{\prime}$ are sequences of the form $e \triangleright_{1} e_{1} \triangleright_{1} \cdots \triangleright_{1} e_{m} \triangleright_{1} e^{\prime}$. The above argument implies that this is an acyclic category and the partial order $\unrhd$ is the partial order associated with this acyclic category.

A flow path does not contain a cycle as in the case of gradient paths, but it can go back to the boundary of one of previous cells. We restrict our attention to the following reduced paths in which such moves are not allowed.

Definition 2.17. A flow path $\gamma=\left(e_{1}, u_{1}, \ldots, e_{n}, u_{n} ; c\right)$ is called reduced if $e_{i+1} \nprec \mu^{-1}\left(u_{i}\right)$ for all $i$. When there is a pair $e_{i+1} \prec \mu^{-1}\left(u_{i}\right)$, we say that $\gamma$ is reducible at $i$. The set of reduced flow paths is denoted by $\overline{\mathrm{FP}}(\mu)$ or $\overline{\mathrm{FP}}(f)$ if $\mu=\mu_{f}$.

For a flow path $\gamma=\left(e_{1}, u_{1}, \ldots, e_{n}, u_{n} ; c\right)$, if $\gamma$ is not reducible at $b-1$ and $e_{b} \prec \mu^{-1}\left(u_{b-1}\right)$, $e_{b} \prec \mu^{-1}\left(u_{b-2}\right), \ldots, e_{b} \prec \mu^{-1}\left(u_{a}\right)$, but $e_{b} \nprec \mu^{-1}\left(u_{a-1}\right)$, the set $\{a, a+1, \ldots, b\}$ is called a reducible interval for $\gamma$.

Lemma 2.18. When a flow path $\gamma=\left(e_{1}, u_{1}, \ldots, e_{n}, u_{n} ; c\right)$ is reducible at $i$, define

$$
r_{i}(\gamma)=\left(e_{1}, u_{1}, \ldots, e_{i-1}, u_{i-1}, e_{i+1}, u_{i+1}, \ldots, e_{n}, u_{n} ; c\right) .
$$

Then $r_{i}(\gamma)$ is a flow path.

Proof. When $\gamma$ is reducible at $i$, we have $u_{i-1} \succ e_{i} \succeq \mu^{-1}\left(u_{i}\right) \succ e_{i+1}$. Hence the condition 4 in the definition of flow path is satisfied.

Definition 2.19. For $\gamma \in \operatorname{FP}(\mu)$ and a reducible interval $I=\{a, a+1, \ldots, b\}$, define

$$
r_{I}(\gamma)=r_{a}\left(r_{a+1}\left(\cdots r_{b}(\gamma) \cdots\right)\right)
$$

Let $I_{1}, \ldots, I_{\ell}$ be the collection of all reducible intervals with $i<j$ for all $i \in I_{p}$ and $j \in I_{p+1}$ and all $p=1, \ldots, \ell-1$. Define

$$
r(\gamma)=r_{I_{1}}\left(r_{I_{2}}\left(\cdots r_{I_{\ell}}(\gamma) \cdots\right)\right)
$$

By Lemma 2.18, $r$ defines a map

$$
r: \mathrm{FP}(\mu) \longrightarrow \overline{\mathrm{FP}}(\mu) .
$$

This is called the reduction map.

We define a relation $\preceq$ on $\operatorname{FP}(\mu)$ as follows.

Definition 2.20. For flow paths $\gamma=\left(e_{1}, u_{1}, \ldots, e_{m}, u_{m} ; c\right)$ and $\gamma^{\prime}=\left(e_{1}^{\prime}, u_{1}^{\prime}, \ldots, e_{n}^{\prime}, u_{n}^{\prime} ; c^{\prime}\right)$, define $\gamma \preceq \gamma^{\prime}$ if and only if there exists a strictly increasing function

$$
\varphi:\{0,1, \ldots, k\} \longrightarrow\{0,1, \ldots, n+1\}
$$

for some $1 \leq k \leq m+1$ satisfying the following conditions: 
1. $\varphi(0)=0$,

2. $u_{j}=u_{\varphi(j)}^{\prime}$ for each $1 \leq j<k$,

3. $\varphi(k)=n+1$, and

4. for each $1 \leq j \leq k, e_{j} \preceq e_{p}^{\prime}$ for all $\varphi(j-1)<p \leq \varphi(j)$.

When $\gamma \preceq \gamma^{\prime}, \gamma$ is called a subpath of $\gamma^{\prime}$. The function $\varphi$ is called the embedding function for $\gamma \preceq \gamma^{\prime}$.

Remark 2.21. Note that we denote $e_{n+1}^{\prime}=c^{\prime}$ for $\gamma^{\prime}=\left(e_{1}^{\prime}, u_{1}^{\prime}, \ldots, e_{n}^{\prime}, u_{n}^{\prime}, c^{\prime}\right)$. Thus the conditions 3 and 4 imply that $e_{k} \preceq c^{\prime}=\tau\left(\gamma^{\prime}\right)$.

In particular, the map $\varphi:\{0,1\} \rightarrow\{0,1, \ldots, n+1\}$ defined by $\varphi(1)=n+1$ is an embedding function for $(\tau(\gamma)) \preceq \gamma$ if $\tau(\gamma) \preceq e_{1}$, where $(\tau(\gamma))$ is the flow path consisting of a single critical cell $\tau(\gamma)$. Another typical example is the following.

Example 2.22. For $\gamma=\left(e_{1}, u_{1}, e_{2}, u_{2}, \ldots, e_{n}, u_{n} ; c\right)$, define

$$
u(\gamma)=\left(u_{1}, u_{1}, e_{2}, u_{2}, \ldots, e_{n}, u_{n} ; c\right) .
$$

The identity map $\{0, \ldots, n+1\} \rightarrow\{0, \ldots, n+1\}$ is an embedding function and we have $\gamma \preceq$ $u(\gamma)$.

In order to understand the meaning of the relation $\preceq$ on $\operatorname{FP}(\mu)$, let us take a look at a more practical example.

Example 2.23. Consider the 2-simplex in Figure 2 with the acyclic partial matching $\mu$ indicated by the arrows. The only critical cell is $c$ with $D(\mu)=\left\{d_{1}, d_{2}, d_{3}\right\}$ and $U(\mu)=\left\{u_{1}, u_{2}, u_{3}\right\}$.

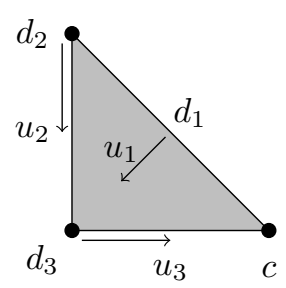

Figure 2: A partial matching on 2-simplex

The sequences

$$
\begin{aligned}
\delta & =\left(d_{1}, u_{1}, u_{2}, u_{2}, d_{3}, u_{3} ; c\right) \\
\gamma & =\left(d_{1}, u_{1}, d_{3}, u_{3} ; c\right)
\end{aligned}
$$

are reduced flow paths terminating at $c$. Let us renumber cells in $\gamma$ as $\gamma=\left(d_{1}^{\prime}, u_{1}^{\prime}, d_{2}^{\prime}, u_{2}^{\prime} ; c\right)$.

Define $\varphi:\{0,1,2,3\} \rightarrow\{0,1,2,3,4\}$ by $\varphi(0)=0, \varphi(1)=1, \varphi(2)=3, \varphi(3)=4$. Then this is an embedding function for $\gamma \prec \delta$. In fact, $u_{\varphi(1)}=u_{1}=u_{1}^{\prime}, u_{\varphi(2)}=u_{3}=u_{2}^{\prime}$ and the condition 2 is satisfied. For the condition $4, d_{1}^{\prime}=d_{1}$ is a face of $d_{1}, d_{2}^{\prime}=d_{3}$ is a face of $u_{2}$ and $d_{3}$. Thus $\gamma \prec \delta$.

In order to prove that $\preceq$ is a partial order on $\operatorname{FP}(\mu)$, we need to prepare a couple of Lemmas. 
Lemma 2.24. For $\gamma \preceq \gamma^{\prime}$ in $\operatorname{FP}(\mu)$, embedding function $\varphi$ in Definition 2.20 is unique.

Proof. Let $\gamma=\left(e_{1}, u_{1}, \ldots, e_{m}, u_{m} ; c\right)$ and $\gamma^{\prime}=\left(e_{1}^{\prime}, u_{1}^{\prime}, \ldots, e_{n}^{\prime}, u_{n}^{\prime} ; c^{\prime}\right)$ be flow paths. Suppose $\gamma \preceq \gamma^{\prime}$ and that there exist two embedding functions

$$
\begin{aligned}
\varphi & :\{0,1, \ldots, k\} \longrightarrow\{0,1, \ldots, n+1\} \\
\varphi^{\prime} & :\left\{0,1, \ldots, k^{\prime}\right\} \longrightarrow\{0,1, \ldots, n+1\}
\end{aligned}
$$

for this relation.

By the condition $u_{j}=u_{\varphi^{\prime}(j)}^{\prime}=u_{\varphi(j)}^{\prime}$, it suffices to show that $k=k^{\prime}$. Suppose $k<k^{\prime}$. By Remark 2.21, we have $e_{k} \preceq c^{\prime}$ by using the conditions for $\varphi$. Let $f$ be a faithful discrete Morse function with $\mu=\mu_{f}$. Since $c^{\prime}$ is critical, we have $f\left(e_{k}\right) \leq f\left(c^{\prime}\right)$. On the other hand, we have $u_{k}=u_{\varphi^{\prime}(k)}^{\prime}$ by the conditions for $\varphi^{\prime}$. By Remark 2.12, we have decreasing sequences

$$
\begin{aligned}
& f\left(e_{1}\right) \geq f\left(u_{1}\right)>\cdots \quad>f\left(e_{m}\right) \geq f\left(u_{m}\right)>f(c) \\
& f\left(e_{1}^{\prime}\right) \geq f\left(u_{1}^{\prime}\right)>\cdots>f\left(e_{n}^{\prime}\right) \geq f\left(u_{n}^{\prime}\right)>f\left(c^{\prime}\right) .
\end{aligned}
$$

In particular, we have

$$
f\left(e_{k}\right) \geq f\left(u_{k}\right)=f\left(u_{\varphi^{\prime}(k)}^{\prime}\right)>f\left(c^{\prime}\right),
$$

which contradicts to $f\left(e_{k}\right) \leq f\left(c^{\prime}\right)$.

An analogous argument implies the following. The proof is omitted.

Lemma 2.25. Let $f$ be a faithful discrete Morse function with $\mu=\mu_{f}$. For $\gamma, \gamma^{\prime} \in \operatorname{FP}(\mu)$, $\gamma \preceq \gamma^{\prime}$ implies $f(\tau(\gamma)) \leq f\left(\tau\left(\left(\gamma^{\prime}\right)\right)\right.$.

Recall that $\ell(\gamma)$ is the length of a flow path $\gamma$.

Lemma 2.26. If $\gamma \preceq \gamma^{\prime}$ and $\tau(\gamma)=\tau\left(\gamma^{\prime}\right)$, then we have $\ell(\gamma) \leq \ell\left(\gamma^{\prime}\right)$.

Proof. Suppose $\gamma=\left(e_{1}, u_{1}, \ldots, e_{m}, u_{m} ; c\right)$ and $\gamma^{\prime}=\left(e_{1}^{\prime}, u_{1}^{\prime}, \ldots, e_{n}^{\prime}, u_{n}^{\prime} ; c\right)$. Let

$$
\varphi:\{0, \ldots, k\} \longrightarrow\{0, \ldots, n+1\}
$$

be the embedding function for $\gamma \preceq \gamma^{\prime}$. Note that we have $e_{k} \prec \tau\left(\gamma^{\prime}\right)=\tau(\gamma)$ by Remark 2.21.

Let $f$ be a faithful discrete Morse function with $\mu=\mu_{f}$. By Lemma 2.25, we have $f(\tau(\gamma)) \leq$ $f\left(e_{k}\right) \leq f\left(\tau\left(\gamma^{\prime}\right)\right)$. Since $\tau(\gamma)=\tau\left(\gamma^{\prime}\right)$, we obtain $f\left(e_{k}\right)=f(\tau(\gamma))$ and the injectivity of $f$ implies that $e_{k}=\tau(\gamma)$. Thus we have $m=k-1$ by the definition of length of $\gamma$. On the other hand, the injectivity of $\varphi$ implies $m+1=k \leq n+1$. And we have $\ell(\gamma) \leq \ell\left(\gamma^{\prime}\right)$.

Remark 2.27. The above proof implies that, when $\tau(\gamma)=\tau\left(\gamma^{\prime}\right)$ and $\gamma \preceq \gamma^{\prime}$, its embedding function $\varphi$ is of the form

$$
\varphi:\{0, \ldots, \ell(\gamma)+1\} \longrightarrow\left\{0, \ldots, \ell\left(\gamma^{\prime}\right)+1\right\} .
$$

Thus when $\tau(\gamma)=\tau\left(\gamma^{\prime}\right)$ and $\ell(\gamma)=\ell\left(\gamma^{\prime}\right)$, the embedding for $\gamma \preceq \gamma^{\prime}$ must be the identity map.

Proposition 2.28. The relation $\preceq$ is a partial order on the set $\operatorname{FP}(\mu)$ of flow paths.

Proof. For $\gamma \in \operatorname{FP}(\mu)$, the relation $\gamma \preceq \gamma$ is given by the identity embedding function.

Suppose $\gamma \preceq \gamma^{\prime}$ and $\gamma^{\prime} \preceq \gamma^{\prime \prime}$. Let

$$
\begin{aligned}
\varphi & :\{0, \ldots, k\} \longrightarrow\left\{0, \ldots, \ell\left(\gamma^{\prime}\right)+1\right\} \\
\varphi^{\prime} & : \quad\left\{0, \ldots, k^{\prime}\right\} \longrightarrow\left\{0, \ldots, \ell\left(\gamma^{\prime \prime}\right)+1\right\}
\end{aligned}
$$


be embedding functions for $\gamma \preceq \gamma^{\prime}$ and $\gamma^{\prime} \preceq \gamma^{\prime \prime}$, respectively.

Since $k^{\prime} \leq \ell\left(\gamma^{\prime}\right)+1$, there exists $r$ such that $\varphi(r-1)<k^{\prime} \leq \varphi(r)$. Define a function $\varphi^{\prime \prime}:\{0, \ldots, r\} \rightarrow\left\{0, \ldots, \ell\left(\gamma^{\prime \prime}\right)+1\right\}$ by

$$
\varphi^{\prime \prime}(i)= \begin{cases}\left(\varphi^{\prime} \circ \varphi\right)(i), & i<r \\ \varphi^{\prime}\left(k^{\prime}\right)=\ell\left(\gamma^{\prime \prime}\right)+1, & i=r .\end{cases}
$$

This is an embedding function for $\gamma \preceq \gamma^{\prime \prime}$.

Finally suppose that $\gamma \preceq \gamma^{\prime}$ and $\gamma^{\prime} \preceq \gamma$ for $\gamma=\left(e_{1}, u_{1}, \ldots, e_{m}, u_{m} ; c\right)$ and $\gamma^{\prime}=\left(e_{1}^{\prime}, u_{1}^{\prime}, \ldots, e_{n}^{\prime}, u_{n}^{\prime} ; c^{\prime}\right)$. By Lemma 2.25 we have $f(\tau(\gamma))=f\left(\tau\left(\gamma^{\prime}\right)\right)$. Since our Morse function $f$ is assumed to be injective, we have $\tau(\gamma)=\tau\left(\gamma^{\prime}\right)$. By Lemma 2.26, we have $\ell(\gamma)=\ell\left(\gamma^{\prime}\right)$.

The relation $\gamma \preceq \gamma^{\prime}$ implies that, if $e_{j}=u_{j}$ in $\gamma$, then

$$
u_{j}=e_{j} \preceq e_{\varphi(j)}^{\prime} \preceq u_{\varphi(j)}^{\prime}=u_{j}
$$

and we have $e_{\varphi(j)}^{\prime}=u_{\varphi(j)}^{\prime}$. Thus we have

$$
\left\{u_{i} \mid e_{i}=u_{i}\right\} \subset\left\{u_{j}^{\prime} \mid e_{j}^{\prime}=u_{j}^{\prime}\right\} .
$$

The assumption $\gamma^{\prime} \preceq \gamma$ then implies that

$$
\left\{u_{i} \mid e_{i}=u_{i}\right\}=\left\{u_{j}^{\prime} \mid e_{j}^{\prime}=u_{j}^{\prime}\right\} .
$$

Furthermore, by Remark 2.27, the embedding function $\varphi$ for the relation $\gamma \preceq \gamma^{\prime}$ is the identity map. Thus the relations $\gamma \preceq \gamma^{\prime}$ and $\gamma^{\prime} \preceq \gamma$ show that the corresponding cells in $\gamma$ and $\gamma^{\prime}$ are identical and we have $\gamma=\gamma^{\prime}$.

We regard the set of reduced flow paths $\overline{\mathrm{FP}}(\mu)$ as a subposet of $\operatorname{FP}(\mu)$. On the other hand, we have the reduction map $r: \mathrm{FP}(f) \rightarrow \overline{\mathrm{FP}}(\mu)$.

Lemma 2.29. The reduction $r: \mathrm{FP}(\mu) \rightarrow \overline{\mathrm{FP}}(\mu)$ is a poset map. It is also a retraction.

Proof. Suppose $\gamma \preceq \delta$ in $\operatorname{FP}(\mu)$ for $\gamma=\left(e_{1}, u_{1}, \ldots, e_{m}, u_{m} ; c\right)$ and $\delta=\left(e_{1}^{\prime}, u_{1}^{\prime}, \ldots, e_{n}^{\prime}, u_{n}^{\prime} ; c^{\prime}\right)$ and let $\varphi:\{0, \ldots, k\} \rightarrow\{0, \ldots, n+1\}$ be the embedding function for $\gamma \preceq \delta$.

Let $I_{1}, \ldots, I_{\ell}$ and $J_{1}, \ldots, J_{\ell^{\prime}}$ be the reducible intervals for $\gamma$ and $\delta$, respectively. Let us first show that the embedding function $\varphi$ can be restricted to

$$
\varphi:\{0, \ldots, k\} \backslash \bigcup_{i=1}^{\ell} I_{i} \longrightarrow\{0, \ldots, n+1\} \backslash \bigcup_{j=1}^{\ell^{\prime}} J_{j} .
$$

In other words, we want to show that, if $\delta$ is reducible at $\varphi(i)$, then $\gamma$ is reducible at $i$. When $\delta$ is reducible at $\varphi(i), e_{\varphi(i)+1}^{\prime} \prec \mu^{-1}\left(u_{\varphi(i)}^{\prime}\right)$. By the conditions for embedding functions, on the other hand, we have $e_{i+1} \prec e_{\varphi(i)+1}^{\prime}$ and $u_{\varphi(i)}^{\prime}=u_{i}$. Thus

$$
e_{i+1} \prec e_{\varphi(i)+1}^{\prime} \prec \mu^{-1}\left(u_{\varphi(i)}^{\prime}\right)=\mu^{-1}\left(u_{i}\right),
$$

which implies that $\gamma$ is reducible at $i$.

Let $k^{\prime}$ be the cardinality of $\{1, \ldots, k\} \backslash \bigcup_{i=1}^{\ell} I_{i}$ and $n^{\prime}=n-\left|J_{1}\right|-\ldots-\left|J_{\ell^{\prime}}\right|$. The order preserving bijections are denoted by

$$
\begin{aligned}
& \psi:\left\{0, \ldots, k^{\prime}\right\} \longrightarrow\{0, \ldots, k\} \backslash \bigcup_{i=1}^{\ell} I_{i} \\
& \theta:\left\{0, \ldots, n^{\prime}+1\right\} \longrightarrow\{0, \ldots, n+1\} \backslash \bigcup_{j=1}^{\ell^{\prime}} J_{j} .
\end{aligned}
$$


Then we have a strictly increasing function $\varphi^{\prime}$ by the composition

$$
\varphi^{\prime}:\left\{0, \ldots, k^{\prime}\right\} \stackrel{\psi}{\longrightarrow}\{0, \ldots, k\} \backslash \bigcup_{i=1}^{\ell} I_{i} \stackrel{\varphi}{\longrightarrow}\{0, \ldots, n+1\} \backslash \bigcup_{j=1}^{\ell^{\prime}} J_{j} \stackrel{\theta^{-1}}{\longrightarrow}\left\{0, \ldots, n^{\prime}+1\right\} .
$$

The fact that $\varphi^{\prime}$ is the embedding function for $r(\gamma) \preceq r(\delta)$ follows immediately from the fact that $\varphi$ is the embedding function for $\gamma \preceq \delta$.

Recall that, when we regard posets as small categories, order preserving maps correspond to functors. The following is an important property of the reduction map from the view point of category theory.

Proposition 2.30. The composition $\mathrm{FP}(\mu) \stackrel{r}{\longrightarrow} \overline{\mathrm{FP}}(\mu) \hookrightarrow \mathrm{FP}(\mu)$ is a descending closure opera$t o r^{2}$.

Proof. Let us show that $r(\gamma) \preceq \gamma$ for any $\gamma \in \operatorname{FP}(\mu)$. Let $I_{1}, \ldots, I_{\ell}$ be the reducible intervals in $\gamma$ and

$$
\psi:\{0,1, \ldots, \ell(r(\gamma))+1\} \longrightarrow\{0, \ldots, n+1\} \backslash \bigcup_{i=1}^{\ell} I_{i}
$$

the order preserving bijection. Then the composition

$$
r(\varphi):\{0,1, \ldots, \ell(r(\gamma))+1\} \stackrel{\psi}{\longrightarrow}\{0, \ldots, n+1\} \backslash \bigcup_{i=1}^{\ell} I_{i} \hookrightarrow\{0,1, \ldots, n+1\}
$$

is the embedding function for $r(\gamma) \preceq \gamma$.

Remark 2.31. The reduction $r(\gamma)$ is the maximal reduced flow path contained in $\gamma$ by Proposition 2.30 .

By Corollary A.22, we obtain the following important fact.

Corollary 2.32. $B \overline{\mathrm{FP}}(\mu)$ is a strong deformation retract of $B \mathrm{FP}(\mu)$.

\subsection{From Combinatorial Flows to Stable Subdivision}

In order to relate the combinatorial definition of flow paths to the homotopy type of $X$, we construct a continuous flow starting from each $x \in X$ by using a faithful discrete Morse function. We also construct a subdivision of $X$ by using those continuous flows.

The first step is to replace characteristic maps for cells as follows.

Proposition 2.33. Given an acyclic partial matching $\mu$ on a finite regular $C W$ complex $X$, we may choose characteristic maps for cells in $X$ in such a way that they satisfy the following conditions: For each matched pair $d \prec \mu(d)=u$ with $\operatorname{dim} u=n$, the characteristic map $\varphi$ for $u$ is a homeomorphism of triples

$$
\varphi:\left(D^{n}, S_{+}^{n-1}, S_{-}^{n-1}\right) \stackrel{\cong}{\longrightarrow}(\bar{u}, \bar{d}, \partial u \backslash d),
$$

where $S_{+}^{n-1}$ and $S_{-}^{n-1}$ are the northern and southern hemispheres of $\partial D^{n}=S^{n-1}$, respectively.

\footnotetext{
${ }^{2}$ See Definition A.21
} 
We need a version of generalized Schönflies theorem to prove this. We first need to recall the notion of locally flat embeddings.

Definition 2.34. Let $M$ and $N$ be topological manifolds without boundaries of dimension $m$ and $n$, respectively. An embedding of $M$ into $N$ is called locally flat at $x \in M$, if there exists a neighborhood $U$ of $x$ in $N$ such that $(U, U \cap M)$ is homeomorphic to $\left(\mathbb{R}^{n}, \mathbb{R}^{m}\right)$.

The following theorem is due to M. Brown [Bro60].

Theorem 2.35. Let $f: S^{n-1} \hookrightarrow S^{n}$ be a locally flat embedding. Then there exists a homeomorphism $\varphi: S^{n} \rightarrow S^{n}$ with

$$
\varphi\left(f\left(S^{n-1}\right)\right)=S^{n-1}=\left\{\left(x_{0}, \ldots, x_{n}\right) \in S^{n} \mid x_{n}=0\right\} .
$$

Any piecewise-linear (PL) embedding of a PL $m$-manifold into a PL $n$-manifold is known to be locally flat, if $n-m \neq 2$. See Theorem 1.7.2 in Rushing's book [Rus73], for example. By the Jordan-Brouwer separation theorem, for any embedding $S^{n-1} \hookrightarrow S^{n}$, the complement of $S^{n-1}$ has two connected components. In particular, we obtain the following fact.

Corollary 2.36. Let $\Sigma^{n-1}$ be a $P L(n-1)$-sphere embedded in a PL n-sphere $\Sigma^{n}$. Let us denote the closures of the connected components of $\Sigma^{n} \backslash \Sigma^{n-1}$ by $\Delta_{+}^{n}$ and $\Delta_{-}^{n}$. Then both $\Delta_{+}^{n}$ and $\Delta_{-}^{n}$ are homeomorphic to an $n$-disk $D^{n}$.

Remark 2.37. Note that this Corollary is not the PL Schönflies theorem, which implies that $\Delta_{+}^{n}$ and $\Delta_{-}^{n}$ are PL disks.

Proof of Proposition 2.33. We modify characteristic maps by induction on dimensions of cells. Suppose we have succeeded in modifying characteristic maps for cells of dimension less than $n$. Let $u$ be an $n$-cell and $\varphi: D^{n} \rightarrow \bar{u}$ the original characteristic map for $u$. Since $X$ is assumed to be regular, the restriction

$$
\left.\varphi\right|_{S^{n-1}}: S^{n-1} \longrightarrow \partial u
$$

is a homeomorphism. The regularity of $X$ also implies that, $\bar{u}$ has a structure of a PL $n$-disk containing $\partial u$ as a PL $(n-1)$-sphere. This can be done by taking the barycentric subdivision of $X$ twice, if necessary.

The boundary $\partial u$ contains a PL $(n-2)$-sphere $\partial d$. By Corollary 2.36, there exist homeomorphisms

$$
\begin{array}{ll}
\varphi_{+}: & D^{n-1} \longrightarrow \bar{d} \\
\varphi_{-} & : \quad D^{n-1} \longrightarrow \partial u \backslash d .
\end{array}
$$

The composition

$$
S^{n-2}=\partial D^{n-1} \stackrel{\varphi_{+}}{\longrightarrow} \partial d \stackrel{\varphi_{-}^{-1}}{\longrightarrow} S^{n-2}
$$

can be extended radially to a homeomorphism $\psi: D^{n-1} \rightarrow D^{n-1}$. The composition

$$
\tilde{\varphi}_{-}: D^{n-1} \stackrel{\psi}{\longrightarrow} D^{n-1} \stackrel{\varphi_{-}}{\longrightarrow} \partial u \backslash d
$$

is a homeomorphism which agrees with $\varphi_{+}$on $S^{n-2}$. Thus we obtain a homeomorphism $\varphi_{\partial}$ : $S^{n-1} \rightarrow \partial u$ by gluing $\varphi_{+}$and $\tilde{\varphi}_{-}$along $S^{n-2}$.

The composition

$$
S^{n-1} \stackrel{\varphi_{\partial}}{\longrightarrow} \partial e \stackrel{\varphi^{-1}}{\longrightarrow} S^{n-1}
$$


is a homeomorphism. Extend this to a homeomorphism $\tilde{\varphi}: D^{n} \rightarrow D^{n}$ radially. Then the composition

$$
\varphi^{\prime}: D^{n} \stackrel{\tilde{\varphi}}{\longrightarrow} D^{n} \stackrel{\varphi}{\longrightarrow} \bar{u}
$$

is a characteristic map for $u$ which satisfies the required condition.

Definition 2.38. Let $d \prec \mu(d)=u$ and $\varphi$ be as above. The complement $\partial u \backslash d$ of $d$ in $\partial u \cong S^{n-1}$ is denoted by $d^{c}$.

The retraction $r: D^{n} \rightarrow S_{-}^{n-1}$ given by $\left(\boldsymbol{x}, x_{n}\right) \mapsto\left(\boldsymbol{x},-\sqrt{1-\|\boldsymbol{x}\|^{2}}\right)$ induces a retraction of $\bar{u}$ onto $d^{c}$ through $\varphi$, which is denoted by $R_{u}: \bar{u} \rightarrow d^{c}$. The composition $\bar{d} \hookrightarrow \bar{u} \stackrel{R_{u}}{\longrightarrow} d^{c}$ is denoted by $R_{d}$.

Remark 2.39. We have the following commutative diagram

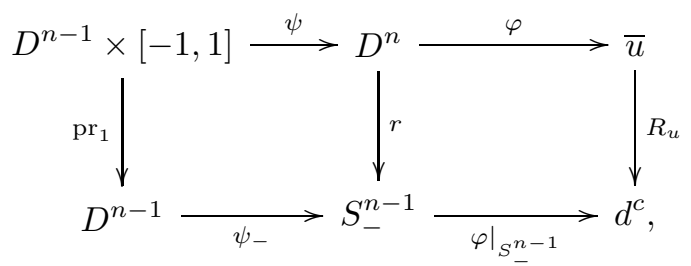

where $\psi$ and $\psi_{-}$are maps defined by

$$
\begin{aligned}
& \psi(\boldsymbol{x}, t)=\left(\boldsymbol{x}, t \sqrt{1-\|\boldsymbol{x}\|^{2}}\right) \\
& \psi_{-}(\boldsymbol{x})=\left(\boldsymbol{x},-\sqrt{1-\|\boldsymbol{x}\|^{2}}\right) .
\end{aligned}
$$

Note that $\left.\psi\right|_{D^{n-1} \times \partial[0,1] \cup \text { Int } D^{n-1} \times[0,1]}$ is a homeomorphism onto $D^{n} \backslash S^{n-2}$. It should be also noted that $R_{d}(A)=A$ if and only if $R_{d}^{-1}(A)=A$ if and only if $A \subset \partial d$. These facts will be used in $\S 3.4$ to construct a subdivision $\operatorname{Sd}_{\mu}(X)$ of $X$.

When $d$ is not a face of any other cell, the deformation retraction $R_{d}$ can be extended to $X \rightarrow X \backslash(d \cup \mu(d))$. Such a deformation retraction is called an elementary collapse in simple homotopy theory [Coh73]. Thus matched pairs can be regarded as generalizations of elementary collapses.

In the rest of this paper, we fix a deformation retraction $R_{u}: \bar{u} \rightarrow d^{c}=\partial u \backslash d$ for each matched pair $d \prec_{1} u$. Now we are ready to construct continuous flows on $X$. Let

$$
L:[-1,1] \times[0,1] \longrightarrow[-1,1]
$$

be the linear flow on $[-1,1]$ which carries $x \in[-1,1]$ to -1 , i.e. $L(x, t)=(1-t) x-t$. By extending this flow, we obtain a flow on $D^{n-1} \times[-1,1]$ and hence a flow $L_{d, u}: \bar{u} \times[0,1] \rightarrow \bar{u}$ which makes the following diagram commutative

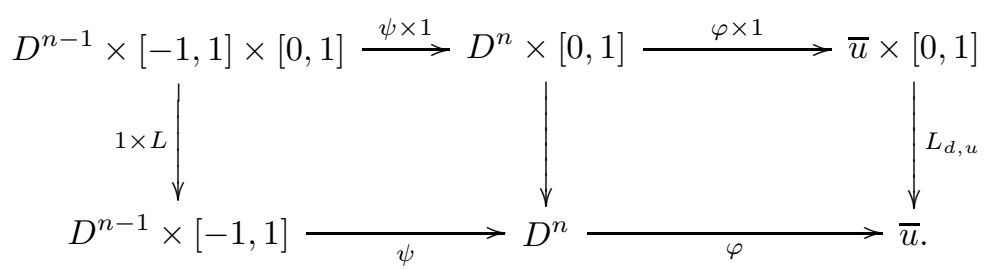

Definition 2.40. Suppose $X$ is finite. For each $x \in X$, we assign a nonnegative number $h_{x}$ and define a continuous path $L_{x}:\left[0, h_{x}\right] \rightarrow X$ with the following properties: 
1. $L_{x}(0)=x$.

2. When $u \cap L_{x}\left(\left[0, h_{x}\right]\right) \neq \emptyset$ for $u \in U(\mu)$, the restriction of $L_{x}$ to $u$ coincides with a restriction of $L_{\mu^{-1}(u), u}$ with a certain parameter shift.

Choose a faithful discrete Morse function $f$ for $\mu$. We proceed by induction on $f(x)$. (Recall that we regard $f$ as an integer-valued locally constant function on $X$.)

When $f(x)$ is the minimum value of $f$, the path $L_{x}$ is defined to be the constant path at $x$ with length 0.

Suppose we have defined paths $L_{y}$ for all points $y$ with $f(y)<f(x)$. Let $e$ be the unique cell containing $x$ in its interior. If $e \in \operatorname{Cr}(\mu)$, we also define $L_{x}$ to be the constant path at $x$ with length 0 . When $e \in U(\mu)$, there exist $y \in \mu^{-1}(e)$ and $s \in[0,1]$ with $x=L_{\mu^{-1}(e), e}(y, s)$. Note that $y$ and $s$ are uniquely determined by $x$. Let $e^{\prime}$ be the unique cell containing $x^{\prime}=L_{\mu^{-1}(e), e}(y, 1)$ in its interior. Then $e^{\prime} \prec e$ but $e^{\prime}$ is not matched with $e$, since $e^{\prime} \neq \mu^{-1}(e)$. Thus $f\left(e^{\prime}\right)<f(e)$, since $f$ is faithful. By the inductive hypothesis, there exist a number $h_{x^{\prime}}$ and a continuous path $L_{x^{\prime}}:\left[0, h_{x^{\prime}}\right] \rightarrow X$ satisfying the required conditions. Define $h_{x}=h_{x^{\prime}}+1-s$ and

$$
L_{x}(t)= \begin{cases}L_{x^{\prime}}(t-1+s), & \text { if } t \in\left[1-s, h_{x}\right] \\ L_{\mu^{-1}(e), e}(y, t+s), & \text { if }[0,1-s] .\end{cases}
$$

When $e \in D(\mu)$, let $e^{\prime}$ be the unique cell containing $x^{\prime}=L_{e, \mu(e)}(x, 1)$ in its interior. Then $f\left(e^{\prime}\right)<f(e)$ and the inductive hypothesis applies to $x^{\prime}$. Now define $h_{x}=h_{x^{\prime}}+1$ and

$$
L_{x}(t)= \begin{cases}L_{x^{\prime}}(t-1), & \text { if } t \in\left[1, h_{x}\right] \\ L_{e, \mu(e)}(x, t), & \text { if }[0,1] .\end{cases}
$$

And we obtain a continuous path

$$
L_{x}:\left[0, h_{x}\right] \longrightarrow X
$$

satisfying the desired conditions. The number $h_{x}$ defined by the above procedure is called the height of $x$ with respect to $f$. And the path $L_{x}$ is called the flow associated to $f$ with initial point $x$.

Remark 2.41. The finiteness of $X$ is used to choose a faithful discrete Morse function $f$ for an acyclic partial matching. If we start from a faithful discrete Morse function, the finiteness assumption is not necessary.

The continuous path constructed above is closely related to flow paths.

Lemma 2.42. For each $x \in X$, there exists a unique reduced flow path $\gamma_{x}$ which contains $x$ in its initial cell $\iota\left(\gamma_{x}\right)$ and contains the image of $L_{x}$ in the union of cells in $\gamma_{x}$.

Conversely, for each reduced flow path $\gamma=\left(e_{1}, u_{1}, \ldots, e_{n}, u_{n} ; c\right)$, there exists $x \in e_{1}$ such that $\gamma_{x}=\gamma$.

Proof. Let $e_{1}, \ldots, e_{n}$ be the sequence of cells appeared in the construction of $L_{x}$. Depending on $e_{i} \in D(\mu)$ or $e_{i} \in U(\mu)$, define $u_{i}=\mu\left(e_{i}\right)$ or $u_{i}=e_{i}$. By the construction, $L_{x}$ lands in a critical cell, say $c$. Then the sequence $\gamma_{x}=\left(e_{1}, u_{1}, \ldots, e_{n}, u_{n} ; c\right)$ is a reduced flow path. The uniqueness follows from the uniqueness of the choices of cells $e_{1}, \ldots, e_{n}$.

Conversely, let $\gamma=\left(e_{1}, u_{1}, \ldots, e_{n}, u_{n} ; c\right)$ be a reduced flow path. Choose a point $z \in c$. Starting with $z$, let us reverse the construction of flows in Definition 2.40 to obtain $x \in X$ with $\gamma_{x}=\gamma$ and $L_{x}\left(h_{x}\right)=z$. We proceed by induction on the length $n$ of $\gamma$. When $n=0, \gamma=(c)$ and there is nothing to prove. 
Suppose we have found such points for reduced flow paths of lengths $\leq n-1$. Let $\gamma^{\prime}=$ $\left(e_{2}, u_{2}, \ldots, e_{n}, u_{n} ; c\right)$ and apply the inductive hypothesis. Then there exists $x^{\prime} \in e_{2}$ such that the image of $L_{x^{\prime}}$ is contained in the union of cells in $\gamma^{\prime}$. Since $\gamma$ is reduced, $e_{2} \subset \partial u_{1} \backslash \overline{\mu^{-1}\left(u_{1}\right)}$. When $e_{1}=\mu^{-1}\left(u_{1}\right)$, there exists $x \in e_{1}$ such that $L_{e_{1}, u_{1}}(x, 1)=x^{\prime}$. When $e_{1}=u_{1}$, there exist $x \in e_{1}$ and $s \in(0,1)$ such that $x^{\prime}=L_{\mu^{-1}\left(u_{1}\right), u_{1}}(x, s)$. By construction, cells containing the image of $L_{x}$ form $\gamma$ and thus $\gamma=\gamma_{x}$.

This lemma allows us to define a surjective map

$$
\pi_{\mu}: X \longrightarrow \overline{\mathrm{FP}}(\mu)
$$

by $\pi_{\mu}(x)=\gamma_{x}$. Hence we have a stratification on $X$

$$
X=\bigcup_{\gamma \in \overline{\mathrm{FP}}(\mu)} e_{\gamma},
$$

where $e_{\gamma}=\pi_{\mu}^{-1}(\gamma)$, for a reduced flow path $\gamma$.

Note that we have a commutative diagram

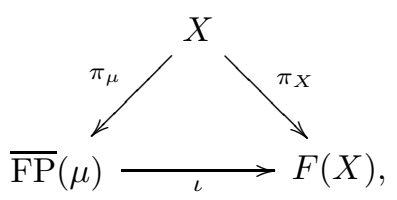

where $\pi_{X}$ is the defining map for the cell decomposition of $X$, i.e. $x \in X$ is mapped to the unique cell containing $x$. Thus

$$
e_{\lambda}=\bigcup_{\iota(\gamma)=e_{\lambda}} e_{\gamma}
$$

for $e_{\lambda} \in F(X)$.

The following description of $e_{\gamma}$ is useful.

Lemma 2.43. For a reduced flow path $\gamma=\left(e_{1}, u_{1}, \ldots, e_{n}, u_{n} ; c\right)$, we have

$$
e_{\gamma}=\left\{x \in e_{1} \mid L_{x}\left(h_{x}\right) \in c\right\} .
$$

Proof. By definition

$$
e_{\gamma}=\left\{x \in e_{1} \mid \gamma_{x}=\gamma\right\} .
$$

The condition $\gamma_{x}=\gamma$ is equivalent to saying that $\gamma$ consists of cells which intersect with the image of $L_{x}$. By the construction of $L_{x}$, this is equivalent to $L_{x}\left(h_{x}\right) \in c$.

Corollary 2.44. For a reduced flow path $\gamma=\left(e_{1}, u_{1}, \ldots, e_{n}, u_{n} ; c\right)$, let $\gamma^{\prime}=\left(e_{2}, u_{2}, \ldots, e_{n}, u_{n} ; c\right)$. Then

$$
e_{\gamma}=R_{u_{1}}^{-1}\left(e_{\gamma^{\prime}}\right) \cap e_{1}
$$

When $e_{1}=\mu^{-1}\left(u_{1}\right)$, we also have

$$
e_{\gamma}=R_{e_{1}}^{-1}\left(e_{\gamma^{\prime}}\right) \cap e_{1}
$$

Proof. Suppose $e_{1}=\mu^{-1}\left(u_{1}\right)$. By the construction of $L_{x}$, we have

$$
e_{\gamma}=\left\{x \in e_{1} \mid L_{e_{1}, u_{1}}(x, 1) \in e_{\gamma^{\prime}}\right\}
$$

and the map $L_{e_{1}, u_{1}}(-, 1): \overline{u_{1}} \rightarrow e_{1}^{c}$ coincides with $R_{u_{1}}$. The case $e_{1}=u_{1}$ is analogous and is omitted. 
The rest of this section is devoted to the proof of the following fact.

Proposition 2.45. The subdivision (2.1) of $X$ is a regular cell decomposition.

In order to prove this, let us consider the following more general situation. Suppose we have a regular cell complex $X$. Suppose further that each cell $e_{\lambda}$ in $X$ is equipped with a decomposition

$$
e_{\lambda}=\bigcup_{\alpha \in A_{\lambda}} e_{\lambda, \alpha} .
$$

We would like to know when the decomposition of $X$

$$
X=\bigcup_{\alpha \in A_{\lambda}} \bigcup_{\lambda \in \Lambda} e_{\lambda, \alpha}
$$

is a regular cell decomposition.

It is easy to see that the closure of $e_{\lambda}$ has the following decomposition

$$
\overline{e_{\lambda}}=\bigcup_{\mu \in \Lambda} \bigcup_{\beta \in A_{\mu}} \bigcup_{e_{\mu, \beta} \cap \bar{e}_{\lambda} \neq \emptyset} e_{\mu, \beta} .
$$

Lemma 2.46. If (2.4) is a regular cell decomposition of $\overline{e_{\lambda}}$ for all $\lambda$, then (2.3) is a regular cell decomposition of $X$, which is a subdivision of the original cell decomposition on $X$.

Proof. By assumption, each $e_{\lambda, \alpha}$ is equipped with a characteristic map

$$
\varphi_{\lambda, \alpha}: D^{\operatorname{dim} e_{\lambda, \alpha}} \longrightarrow \overline{e_{\lambda}} .
$$

Composed with the inclusion $\overline{e_{\lambda}} \hookrightarrow X$, we obtain a characteristic map for $e_{\alpha, \lambda}$ in $X$, since the closure of $e_{\lambda, \mu}$ in $\overline{e_{\lambda}}$ coincides with the closure in $X$.

The condition that $\partial e_{\lambda, \alpha}=\overline{e_{\lambda, \alpha}} \backslash e_{\lambda, \alpha}$ is covered with cells of dimension $<\operatorname{dim} e_{\lambda, \alpha}$ follows from the assumption.

The following observation is essential in the proof of Proposition 2.45.

Lemma 2.47. Let $K$ be a triangulation of a convex polytope $P$ of dimension $n$. Define a relation $\sim$ on $P \times[0,1]=|K| \times[0,1]$ by $(\boldsymbol{x}, s) \sim(\boldsymbol{x}, t)$ for $\boldsymbol{x} \in \partial P$ and $s, t \in[0,1]$. The equivalence relation generated by $\sim$ is also denoted by $\sim$. Denote the quotient space $P \times[0,1] / \sim$ by $E$ and the canonical projection onto $P$ by

$$
p: E \longrightarrow P \text {. }
$$

Then for any simplex $\sigma \in K, p^{-1}(\sigma)$ is homeomorphic to $D^{\operatorname{dim} \sigma+1}$.

More generally for any subcomplex $L \subset K$ whose geometric realization is homeomorphic to a disk of dimension $n, p^{-1}(|L|)$ is homeomorphic to a disk of dimension $n+1$.

Proof. When $\sigma \cap \partial P=\emptyset, p^{-1}(\sigma)$ can be identified with $\sigma \times[0,1]$ and is homeomorphic to $D^{\operatorname{dim} \sigma+1}$. Suppose $\sigma \cap \partial P \neq \emptyset$. Then $\tau=\sigma \cap \partial P$ is a face of $\sigma$. We have

$$
p^{-1}(\sigma)=\sigma \times[0,1] / \sim_{\sigma},
$$

where the equivalence relation $\sim_{\sigma}$ is the restriction of $\sim$ and thus $(\boldsymbol{x}, s) \sim_{\sigma}(\boldsymbol{x}, t)$ for $\boldsymbol{x} \in \sigma \cap \partial P$ and $s, t \in[0,1]$.

Let $\left\{v_{0}, v_{1}, \ldots, v_{k}\right\}$ be the set of vertices of $\sigma$. We may assume that the vertices belonging to $\tau$ are the first $\ell+1$ vertices $v_{0}, \ldots, v_{\ell}$. Define a convex polytope $Q$ contained in $\sigma \times[0,1]$ by

$$
Q=\operatorname{Conv}\left(\left(v_{0}, 0\right), \ldots,\left(v_{\ell}, 0\right),\left(v_{\ell+1}, 0\right), \ldots,\left(v_{k}, 0\right),\left(v_{\ell+1}, 1\right), \ldots,\left(v_{k}, 1\right)\right) .
$$


We claim that $p^{-1}(\sigma)$ is homeomorphic to $Q$. A homeomorphism $\pi: p^{-1}(\sigma) \rightarrow Q$ is, for example, defined by

$$
\pi\left(\left[\sum_{i=0}^{k} t_{i} v_{i}, s\right]\right)=\sum_{i=0}^{\ell} t_{i}\left(v_{i}, 0\right)+\sum_{i=\ell+1}^{k}(1-s) t_{i}\left(v_{i}, 0\right)+\sum_{i=\ell+1}^{k} s t_{i}\left(v_{i}, 1\right) .
$$

Since elements in the face $\tau$ correspond to $\sum_{i=0}^{k} t_{i} v_{i}$ with $t_{\ell+1}=\cdots=t_{k}=0$, this map is well defined and continuous. And this is easily seen to be bijective.

Let $L$ be a subcomplex of $K$ with $|L| \cong D^{n}$. We prove that $p^{-1}(|L|)$ is homeomorphic to an $(n+1)$-disk by induction on the number of $n$-simplices and $n$. Suppose we have proved the statement for $n \leq m-1$. Furthermore suppose that we have proved the statement for subcomplexes of dimension $m$ and the number of $m$-simplices less than $k$.

Suppose $L$ has $k m$-simplices. Choose an $m$-simplex $\sigma$ in $L$. Let $L^{\prime}$ be the subcomplex of $L$ obtained by removing $\sigma$ and its faces in $\partial|L|$. Since $|L|$ is homeomorphic to a disk, it is collapsible to a point. Thus we may choose $\sigma$ with which $\left|L^{\prime}\right|$ is homeomorphic to an $m$-disk. Then $\sigma \cap\left|L^{\prime}\right|$ is homeomorphic to an $(m-1)$-disk. By inductive assumption on dimension, $p^{-1}\left(\sigma \cap\left|L^{\prime}\right|\right)$ is homeomorphic to an $m$-disk. By inductive assumption on the number of $m$-cells, $p^{-1}\left(\left|L^{\prime}\right|\right)$ is homeomorphic to an $(m+1)$-disk. Thus $p^{-1}(|L|)=p^{-1}\left(\left|L^{\prime}\right|\right) \cup p^{-1}(\sigma)$ is homeomorphic to an $(m+1)$-disk as a union of two $(m+1)$-disks along an $m$-disk.

Proof of Proposition 2.45. Let us verify the condition in Lemma 2.46. By the diagram (2.2), we have

$$
e_{\lambda}=\bigcup_{\iota(\gamma)=e_{\lambda}} e_{\gamma}
$$

for a cell $e_{\lambda}$ in $F(X)$, which leads to a decomposition of $X$

$$
X=\bigcup_{\lambda \in F(X) \iota(\gamma)=e_{\lambda}} e_{\gamma}
$$

We need to construct a characteristic map for each $e_{\gamma}$ and prove that

$$
\overline{e_{\lambda}}=\bigcup_{\gamma} \bigcup_{e_{\gamma} \cap \overline{e_{\lambda}} \neq \emptyset} e_{\gamma}
$$

is a regular cell decomposition of $\overline{e_{\lambda}}$.

Let us first construct a characteristic map $\varphi_{\gamma}$ for $e_{\gamma}$. Choose a faithful discrete Morse function $f$ with $\mu=\mu_{f}$. We construct $\varphi_{\gamma}$ by induction on $f(\iota(\gamma))$.

When $f(\iota(\gamma))$ is the minimum value of $f, \iota(\gamma)$ is critical and $\gamma$ is the constant flow path $(\iota(\gamma))$. The characteristic map $\varphi_{\gamma}$ for $e_{\gamma}=\iota(\gamma)$ is defined to be the characteristic map for $\iota(\gamma)$.

Suppose we have constructed a characteristic map of $e_{\gamma}$ for $\gamma$ with $f(\iota(\gamma)) \leq k$. Let $\gamma=$ $\left(e_{1}, u_{1}, \ldots, e_{n}, u_{n} ; c\right)$ be a reduced flow path with $f\left(e_{1}\right)=k+1$. (Recall that our $\bar{f}$ is $\mathbb{Z}$-valued.) Since $\gamma$ is nontrivial, $e_{1}$ is not critical. Let $\gamma^{\prime}=\left(e_{2}, u_{2}, \ldots, e_{n}, u_{n} ; c\right)$. Let $\varphi: D^{n} \rightarrow \overline{u_{1}}$ be the characteristic map for $u_{1}$. By Proposition 2.33, we may assume that $\varphi$ is a homeomorphism of triples

$$
\varphi:\left(D^{n}, S_{+}^{n-1}, S_{-}^{n-1}\right) \longrightarrow\left(\overline{u_{1}}, \overline{d_{1}}, d_{1}^{c}\right)
$$


where $d_{1}=\mu^{-1}\left(u_{1}\right)$. By Remark 2.39, we have a commutative diagram

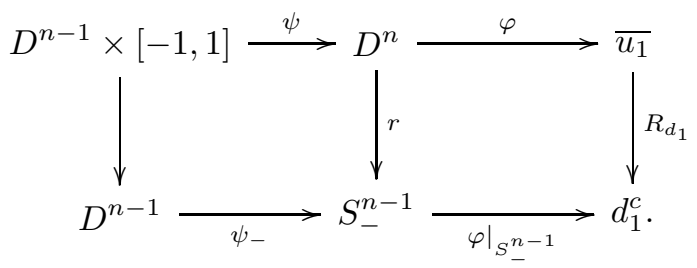

(See Definition 2.38 and Remark 2.39 for definitions of $r, \psi$, and $\psi_{-}$.)

By Remark 2.12, we have

$$
k+1=f\left(e_{1}\right) \geq f\left(u_{1}\right)>f\left(e_{2}\right)=f\left(\iota\left(\gamma^{\prime}\right)\right)
$$

and the inductive hypothesis applies to $\gamma^{\prime}$. Let

$$
\varphi_{\gamma^{\prime}}: D^{\operatorname{dim} e_{\gamma^{\prime}}} \longrightarrow \overline{e_{\gamma^{\prime}}}
$$

be the characteristic map for $e_{\gamma^{\prime}}$. Note that $e_{\gamma^{\prime}} \subset \partial u_{1} \backslash d_{1}$ by construction. Since $\left.\varphi\right|_{S_{-}^{n-1}}$ is a homeomorphism, there exists an embedding $\alpha: D^{\operatorname{dim} e_{\gamma^{\prime}}} \hookrightarrow D^{n-1}$ making the following diagram commutative

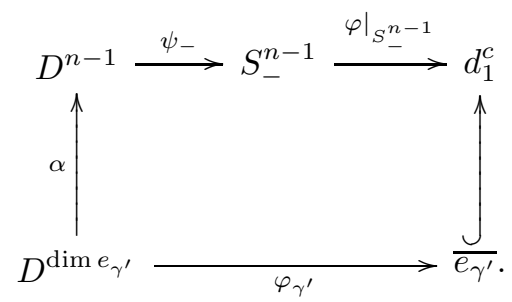

There are two cases; $e_{1}=d_{1}$ or $e_{1}=u_{1}$.

The case $e_{1}=d_{1}$ : We have $e_{\gamma}=R_{d_{1}}^{-1}\left(e_{\gamma^{\prime}}\right) \cap d_{1}$ by Corollary 2.44. Let $i_{0}: D^{n-1} \hookrightarrow D^{n-1} \times$ $[-1,1]$ be the inclusion into $D^{n-1} \times\{1\}$. Then the composition

$$
D^{\operatorname{dim} e_{\gamma^{\prime}}} \stackrel{\alpha}{\longrightarrow} D^{n-1} \stackrel{i_{0}}{\longrightarrow} D^{n-1} \times[-1,1] \stackrel{\psi}{\longrightarrow} D^{n} \stackrel{\varphi}{\longrightarrow} \overline{e^{n}} \hookrightarrow X
$$

is a characteristic map for $e_{\gamma}$.

The case $e_{1}=u_{1}$ : In this case $e_{\gamma}=R_{u_{1}}^{-1}\left(e_{\gamma^{\prime}}\right) \cap u_{1}$. By pulling back the regular cell decomposition of $d_{1}^{c}$ via $\varphi, S_{-}^{n-1}$ has a structure of regular cell complex. Let $K$ be a simplicial subdivision of this regular cell decomposition. Then the map $\psi$ can be regarded as the quotient map

$$
|K| \times[0,1] \longrightarrow|K| \times[0,1] / \sim
$$

in Lemma 2.47. Thus, by Lemma 2.47, $\varphi^{-1}\left(R_{u_{1}}^{-1}\left(e_{\gamma^{\prime}}\right)\right)$ is homeomorphic to a disk of dimension $\operatorname{dim} e_{\gamma^{\prime}}+1$ and we obtain a characteristic map for $e_{\gamma}$.

The fact that $\overline{e_{\gamma}} \backslash e_{\gamma}$ is covered with cells of dimension $<\operatorname{dim} e_{\gamma}$ follows from the construction of the characteristic map for $e_{\gamma}$, or the construction of a homeomorphism in Lemma 2.47.

Definition 2.48. The decomposition (2.1) is called the stable subdivision of $X$ associated to $\mu$ and is denoted by $\operatorname{Sd}_{\mu}(X)$ or $\operatorname{Sd}_{f}(X)$ when $\mu=\mu_{f}$ for a faithful discrete Morse function $f$. 
For a critical cell $c$, the subspace

$$
W^{s}(c)=\bigcup_{\tau(\gamma)=c} e_{\gamma}
$$

is called the stable subspace of $c$.

Remark 2.49. Recall that a smooth Morse function on a manifold $M$ induces a decomposition of $M$ by stable manifolds. The stable manifold $W^{s}(c)$ at a critical point $c$ is a submanifold of $M$ consisting of points on gradient flows going down to $c$. The stable subdivision defined above is a discrete analogue of this decomposition.

Example 2.50. Consider the cubical cell decomposition of a torus $T^{2}$ given by the left picture in Figure 3. Define an acyclic partial matching on this cell complex by the right picture in Figure 3 , in which critical cells are drawn by black.
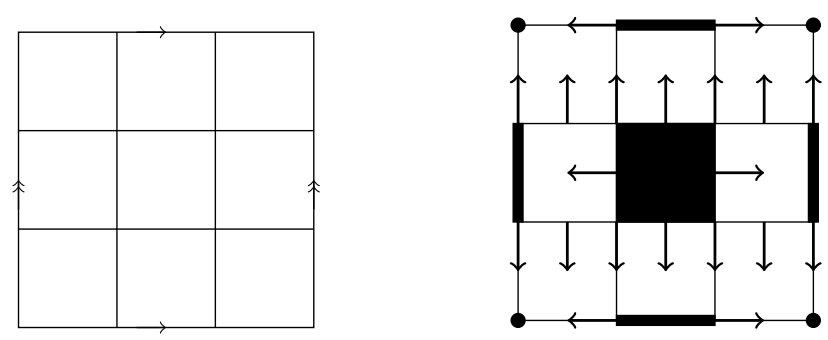

Figure 3: An acyclic partial matching on $T^{2}$

The decomposition by stable subspaces and the stable subdivision are drawn in Figure 4 .
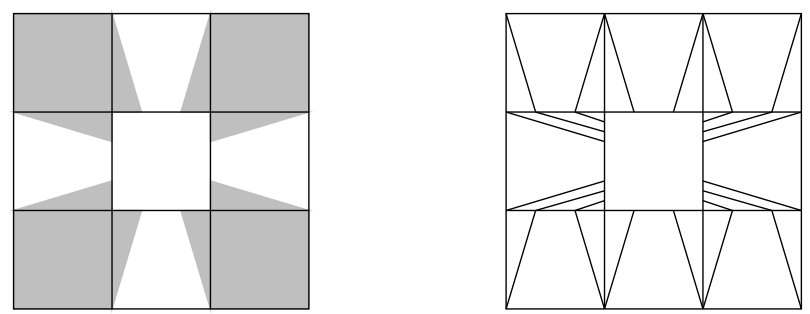

Figure 4: Stable subdivision on $T^{2}$

\section{The Flow Category}

The aim of this section is to construct a poset-enriched category $C(\mu)$, called the flow category, having critical cells of $\mu$ as objects and show that the classifying space of $C(\mu)$ is homotopy equivalent to $X$. 


\subsection{Small Categories}

Let us first fix notation and terminology for categories and functors. We regard a small category as a monoid in the category of quivers.

\section{Definition 3.1.}

1. A quiver $Q$ consists of a set $Q_{0}$ of vertices and a set $Q_{1}$ of arrows together with maps

$$
s, t: Q_{1} \longrightarrow Q_{0}
$$

For $x, y \in Q_{0}$ and $u \in Q_{1}$, when $s(u)=x$ and $t(u)=y$, we write $u: x \rightarrow y$. By definition, the set of arrows from $x$ to $y$ equals $s^{-1}(x) \cap t^{-1}(y)$ and is denoted by $Q(x, y)$.

2. For a quiver $Q$, define

$$
N_{2}(Q)=\left\{(u, v) \in Q_{1}^{2} \mid s(u)=t(v)\right\}
$$

3. A (small) category is a quiver $C$ equipped with maps

$$
\begin{array}{ll}
\circ & : \quad N_{2}(C) \longrightarrow C_{1} \\
\iota & : \quad C_{0} \longrightarrow C_{1}
\end{array}
$$

satisfying the associativity and the unit conditions, i.e.

$$
\begin{aligned}
(u \circ v) \circ w & =u \circ(v \circ w) \\
u & =\iota(t(u)) \circ u=u \circ \iota(s(u))
\end{aligned}
$$

for all $u, v, w \in C_{1}$ with $s(u)=t(v)$ and $s(v)=t(w)$. We also require that $s(u \circ v)=s(v)$, $t(u \circ v)=t(u)$, and $s(\iota(x))=t(\iota(x))=x$.

For $x \in C_{0}, \iota(x)$ is denoted by $1_{x}$ and is called the identity morphism at $x$.

4. A category $C$ is said to be acyclic if either $C(x, y)$ or $C(y, x)$ is empty for any $x, y \in C_{0}$ with $x \neq y$ and $C(x, x)=\left\{1_{x}\right\}$ for any $x \in C_{0}$.

Definitions of functors and natural transformations in this formulation should be obvious and are omitted.

Remark 3.2. A poset is a typical example of an acyclic category. Many of properties and constructions for posets can be extended to acyclic categories.

For a small category $C$, we have a coproduct decomposition

$$
C_{1}=\coprod_{x, y \in C_{0}} C(x, y)
$$

Thus a small category $C$ can be defined as a collection of a set $C_{0}$, a family of sets $\{C(x, y)\}_{x, y \in C_{0}}$, maps

$$
\circ: C(y, z) \times C(x, y) \longrightarrow C(x, z),
$$

and elements $1_{x} \in C(x, x)$ satisfying the associativity and the unit conditions.

In order to study higher structures, we need categories whose sets of morphisms $C(x, y)$ are topological spaces, posets, small categories, and so on. More generally, there is a notion of categories enriched over a monoidal category. Recall that, roughly speaking, a monoidal category is a category equipped with a "tensor product operation" for pairs of objects. 
Definition 3.3. Let $(\boldsymbol{V}, \otimes, 1)$ be a strict monoidal category ${ }^{3}$.

1. A $\boldsymbol{V}$-quiver $Q$ consists of a set $Q_{0}$ of vertices and a family $\{Q(x, y)\}_{x, y \in Q_{0}}$ of objects in $\boldsymbol{V}$.

2. A category enriched over $\boldsymbol{V}$ or simply a $\boldsymbol{V}$-category consists of a $\boldsymbol{V}$-quiver $C$ together with

- a morphism

$$
\circ: C(y, z) \otimes C(x, y) \longrightarrow C(x, z)
$$

in $\boldsymbol{V}$ for each triple $x, y, z \in C_{o}$ and

- a morphism $1_{x}: 1 \rightarrow C(x, x)$ in $\boldsymbol{V}$ for each $x \in C_{0}$

that are subject to the standard requirements of being a small category.

Examples of monoidal categories include the category Poset of posets, the category Top of topological spaces, and the category Cat of small categories. Monoidal structures are given by products in all of these categories. We use the following terminologies.

Definition 3.4. Categories enriched over Poset, Top and Cat are called poset-categories, topological categories, and (strict) 2-categories respectively.

Remark 3.5. Note that poset-categories can be regarded as 2-categories under the inclusion Poset $\subset$ Cat.

Our first task in this section is to construct a poset-category $C(\mu)$ from an acyclic partial matching $\mu$. Since poset-categories are 2-categories, we need to recall basic definitions and properties of 2-categories.

Definition 3.6. For a 2-category $C$ and objects $x, y \in C_{0}, C(x, y)$ is a small category. Thus it consists of the set of objects $C(x, y)_{0}$ and morphisms $C(x, y)_{1}$. Elements of $C(x, y)_{0}$ and $C(x, y)_{1}$ are called 1-morphisms and 2-morphisms in $C$, respectively. When $\theta \in C(x, y)(u, v)$, we write $\theta: u \Rightarrow v$.

The classes of all 1-morphisms and 2-morphisms in $C$ are denoted by $C_{1}$ and $C_{2}$, respectively. When $C_{2}$ is a set, $C$ is called a small 2-category.

There are two kinds of compositions in a 2-category $C$. First of all, given a triple $x, y, z \in C_{0}$ of objects, we have the horizontal composition functor

$$
\circ: C(y, z) \times C(x, y) \longrightarrow C(x, z) .
$$

On the other hand, for a triple $u, v, w \in C(x, y)_{0}$, we have the vertical composition denoted by

$$
\text { * : } C(x, y)(v, w) \times C(x, y)(u, v) \longrightarrow C(x, y)(u, w) .
$$

There are weaker versions of 2-categories such as bicategories and $(\infty, 2)$-categories. Although we only use strict 2-categories, we are often forced to use weaker notions of functors between 2-categories.

Definition 3.7. Let $C$ and $D$ be small 2-categories. A colax functor $f$ from $C$ to $D$ consists of the following data:

- a map

$$
f: C_{0} \longrightarrow D_{0}
$$

\footnotetext{
${ }^{3}$ It is well known that any monoidal category can be replaced by a strict one. See Mac Lane's book [ML98] or Kassel's book [Kas95], for example.
} 
- a family of functors

$$
f=f_{y, x}: C(x, y) \longrightarrow D(f(x), f(y)),
$$

- a family of natural transformations

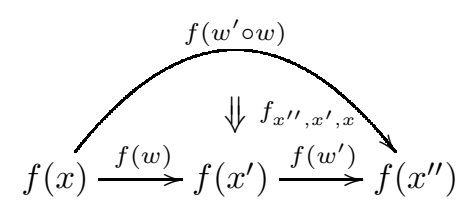

- a family of natural transformations

$$
f(x) \frac{f\left(1_{x}\right)}{\Downarrow_{f_{f}(x)}} f(x) .
$$

These maps are subject to the following additional conditions:

1. The following diagram is commutative in $D(f(x), f(t))$

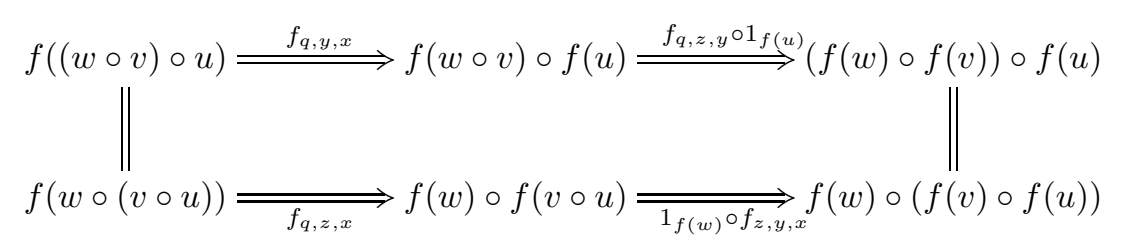

for any composable 1-morphisms $x \stackrel{u}{\longrightarrow} y \stackrel{v}{\longrightarrow} z \stackrel{w}{\longrightarrow} q$ in $C$.

2. The following diagrams are commutative in $D(f(x), f(y))$

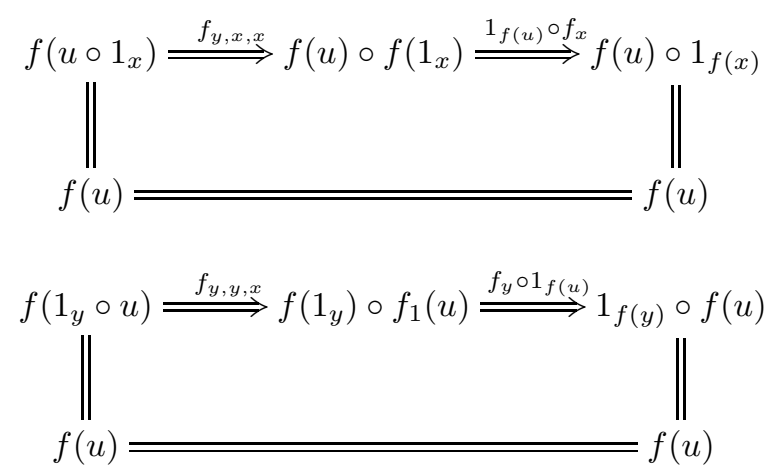

for any 1-morphism $u: x \rightarrow y$ in $C$.

$f$ is called a normal colax functor when $f_{x}$ is the identity for all objects $x \in C_{0}$.

For simplicity, we suppress the indices in $f_{z, y, x}$ and $f_{x}$ and denote them by $f$, when there is no danger of confusion. 
Remark 3.8. Note that colax functors are often called oplax functors in the literature. The choice of the term "colax" is based on the general principle that, when 2-morphisms are reversed, we put a suffix "co".

Remark 3.9. We may reverse the directions of natural transformations $f_{x, y, z}$ and $f_{x}$ to obtain the notion of lax functors.

When these natural transformations are isomorphisms, $f$ is called a pseudofunctor. In particular, a pseudofunctor can be regarded as a lax functor and a colax functor.

\subsection{Categories of Combinatorial Flows}

Here we construct a poset category $C(\mu)$ out of the poset of flow paths $\operatorname{FP}(\mu)$. A reduced version $\bar{C}(\mu)$ is also introduced.

We first define poset-enriched quivers ${ }^{4} Q(\mu)$ and $\bar{Q}(\mu)$.

Definition 3.10. Define $Q(\mu)_{0}=\operatorname{Cr}(\mu)$ and

$$
Q(\mu)_{1}=\{(c, \gamma) \in \operatorname{Cr}(\mu) \times \operatorname{FP}(\mu) \mid c \succ \iota(\gamma)\} .
$$

Extend the target map $\tau: \operatorname{FP}(\mu) \rightarrow \operatorname{Cr}(\mu)$ to a structure of quiver

$$
\sigma, \tau: Q(\mu)_{1} \longrightarrow Q(\mu)_{0}
$$

by $\sigma(c, \gamma)=c$. Similarly we define $\bar{Q}(\mu)_{0}=Q(\mu)_{0}=\operatorname{Cr}(\mu)$ and

$$
\bar{Q}(\mu)_{1}=\{(c, \gamma) \in \operatorname{Cr}(\mu) \times \overline{\mathrm{FP}}(\mu) \mid c \succ \iota(\gamma)\} .
$$

The source and the target maps $\sigma, \tau: \bar{Q}(\mu)_{1} \rightarrow \bar{Q}(\mu)_{0}$ are defined analogously.

For each pair $\left(c, c^{\prime}\right)$ of critical cells, the forgetful map

$$
Q(\mu)\left(c, c^{\prime}\right) \longrightarrow \mathrm{FP}(\mu)
$$

is injective. Define a partial order on $Q(\mu)\left(c, c^{\prime}\right)$ as a full subposet of $\operatorname{FP}(\mu)$ under this injection. The poset structure on $\bar{Q}(\mu)\left(c, c^{\prime}\right)$ is induced analogously from that of $\overline{\mathrm{FP}}(\mu)$.

The resulting poset-quivers $Q(\mu)$ and $\bar{Q}(\mu)$ are called the flow quiver and the reduced flow quiver of $\mu$, respectively. When $\mu=\mu_{f}$ for a discrete Morse function $f$, they are also denoted by $Q(f)$ and $\bar{Q}(f)$, respectively.

Remark 3.11. When $\gamma=\left(e_{1}, u_{1}, \ldots, e_{n}, u_{n} ; c^{\prime}\right)$, we denote $(c, \gamma) \in Q(\mu)\left(c, c^{\prime}\right)$ by $\left(c ; e_{1}, u_{1}, \ldots, e_{n}, u_{n} ; c^{\prime}\right)$ or simply by $\gamma$ when there is no danger of confusion.

In order to make these quivers into small categories, we need to specify how to compose morphisms. For the flow quiver $Q(\mu)$, it is simply given by concatenations.

Definition 3.12. For $\gamma \in Q(\mu)\left(c_{1}, c_{2}\right)$ and $\gamma^{\prime} \in Q(\mu)\left(c_{2}, c_{3}\right)$, define $\gamma * \gamma^{\prime}$ by

$$
\gamma * \gamma^{\prime}=\left(c_{1} ; e_{1}, u_{1}, \ldots, e_{m}, u_{m}, e_{1}^{\prime}, u_{1}^{\prime}, \ldots, e_{n}^{\prime}, u_{n}^{\prime} ; c_{3}\right)
$$

when $\gamma=\left(c_{1} ; e_{1}, u_{1}, \ldots, e_{m}, u_{m} ; c_{2}\right)$ and $\gamma^{\prime}=\left(c_{2} ; e_{1}^{\prime}, u_{1}^{\prime}, \ldots, e_{n}^{\prime}, u_{n}^{\prime} ; c_{3}\right)$.

Remark 3.13. The fact that $\gamma * \gamma^{\prime}$ belongs to $Q(\mu)\left(c_{1}, c_{3}\right)$ can be verified as follows. Suppose $\gamma=\left(c_{1} ; e_{1}, u_{1}, \ldots, e_{m}, u_{m} ; c_{2}\right)$ and $\gamma^{\prime}=\left(c_{2} ; e_{1}^{\prime}, u_{1}^{\prime}, \ldots, e_{n}^{\prime}, u_{n}^{\prime} ; c_{3}\right)$. By definition, we have $u_{m} \succ c_{2}$ and $c_{2} \succ e_{1}^{\prime}$. Thus $u_{m} \succ e_{1}^{\prime}$ and we obtain a flow path $\gamma * \gamma^{\prime}$.

\footnotetext{
${ }^{4}$ Definition 3.3 .
} 
Lemma 3.14. The concatenation of flow paths defines a poset map

$$
*: Q(\mu)\left(c_{1}, c_{2}\right) \times Q(\mu)\left(c_{2}, c_{3}\right) \longrightarrow Q(\mu)\left(c_{1}, c_{3}\right),
$$

where $Q(\mu)\left(c_{1}, c_{2}\right) \times Q(\mu)\left(c_{2}, c_{3}\right)$ is equipped with the product poset structure.

Proof. Suppose that $\left(\gamma, \gamma^{\prime}\right)$ and $\left(\delta, \delta^{\prime}\right)$ in $Q(\mu)\left(c_{1}, c_{2}\right) \times Q(\mu)\left(c_{2}, c_{3}\right)$ satisfy the order relation $\left(\gamma, \gamma^{\prime}\right) \preceq\left(\delta, \delta^{\prime}\right)$. In other words, $\gamma \preceq \delta$ and $\gamma^{\prime} \preceq \delta^{\prime}$ hold in $Q(\mu)\left(c_{1}, c_{2}\right)$ and $Q(\mu)\left(c_{2}, c_{3}\right)$, respectively.

Suppose

$$
\begin{aligned}
\gamma & =\left(c_{1} ; e_{1}, u_{1}, \ldots, e_{m}, u_{m} ; c_{2}\right) \\
\gamma^{\prime} & =\left(c_{1} ; e_{m+1}^{\prime}, u_{m+1}^{\prime}, \ldots, e_{m+m^{\prime}}^{\prime}, u_{m+m^{\prime}}^{\prime} ; c_{3}\right) \\
\delta & =\left(c_{2} ; e_{1}^{\prime \prime}, u_{1}^{\prime \prime}, \ldots, e_{n}^{\prime \prime}, u_{n}^{\prime \prime} ; c_{2}\right) \\
\delta^{\prime} & =\left(c_{2} ; e_{n+1}^{\prime \prime \prime}, u_{n+1}^{\prime \prime \prime}, \ldots, e_{n+n^{\prime}}^{\prime \prime \prime}, u_{n+n^{\prime}}^{\prime \prime \prime} ; c_{3}\right) .
\end{aligned}
$$

By Remark 2.27, the embedding functions for the relations $\gamma \preceq \delta$ and $\gamma^{\prime} \preceq \delta^{\prime}$ are of the following form

$$
\begin{aligned}
\varphi & : \quad\{0, \ldots, m+1\} \rightarrow\{0, \ldots, n+1\} \\
\varphi^{\prime} & : \quad\left\{0, \ldots, m^{\prime}+1\right\} \rightarrow\left\{0, \ldots, n^{\prime}+1\right\}
\end{aligned}
$$

According to our numbering scheme, the conditions for $\gamma \preceq \delta$ are

1. $\varphi(0)=0$,

2. $u_{j}=u_{\varphi(j)}^{\prime \prime}$ for $1 \leq j<m+1$,

3. $\varphi(m+1)=n+1$,

4. for each $1 \leq j \leq m+1, e_{j} \preceq e_{p}^{\prime \prime}$ for all $\varphi(j-1)<p \leq \varphi(j)$

and the conditions for $\gamma^{\prime} \preceq \delta^{\prime}$ are

1. $\varphi^{\prime}(0)=0$,

2. $u_{j}^{\prime}=u_{\varphi^{\prime}(j-m)+n}^{\prime \prime \prime}$ for $m+1 \leq j<m+m^{\prime}+1$,

3. $\varphi^{\prime}\left(m^{\prime}+1\right)=n^{\prime}+1$,

4. for each $m+1 \leq j \leq m+m^{\prime}+1, e_{j} \preceq e_{p}^{\prime \prime}$ for all $\varphi^{\prime}(j-1-m)+n<p \leq \varphi^{\prime}(j-m)+n$.

Now define a map

$$
\varphi * \varphi^{\prime}:\left\{0, \ldots, m+m^{\prime}+1\right\} \rightarrow\left\{0, \ldots, n+n^{\prime}+1\right\}
$$

by

$$
\left(\varphi * \varphi^{\prime}\right)(i)= \begin{cases}\varphi(i), & 0 \leq i \leq m \\ \varphi^{\prime}(i-m)+n, & m+1 \leq i \leq m+m^{\prime}+1 .\end{cases}
$$

Let us verify that this map gives rise to the relation $\gamma * \gamma^{\prime} \preceq \delta * \delta$. The conditions $\left(\varphi * \varphi^{\prime}\right)(0)=0$ and $\left(\varphi * \varphi^{\prime}\right)\left(m+m^{\prime}+1\right)=n+n^{\prime}+1$ are immediate from the definition. The remaining conditions in Definition 2.20 can be split into the following: 
1. $u_{j}=u_{\varphi * \varphi^{\prime}(j)}^{\prime \prime}$ for $1 \leq j<m+1$,

2. $u_{j}^{\prime}=u_{\varphi * \varphi(j)}^{\prime \prime \prime}$ for $m+1 \leq j<m+m^{\prime}+1$,

3. for each $1 \leq j \leq m+1, e_{j} \preceq e_{p}^{\prime \prime}$ for all $\left(\varphi * \varphi^{\prime}\right)(j-1)<p \leq\left(\varphi * \varphi^{\prime}\right)(j)$, and

4. for each $m+1 \leq j \leq m+m^{\prime}+1, e_{j}^{\prime} \preceq e_{p}^{\prime \prime \prime}$ for all $\left(\varphi * \varphi^{\prime}\right)(j-1)<p \leq\left(\varphi * \varphi^{\prime}\right)(j)$.

By the definition of $\varphi * \varphi^{\prime}$, these conditions are

1. $u_{j}=u_{\varphi(j)}^{\prime \prime}$ for $1 \leq j<m+1$,

2. $u_{j}^{\prime}=u_{\varphi^{\prime}(j-m)+n}^{\prime \prime \prime}$ for $m+1 \leq j<m+m^{\prime}+1$,

3. for each $1 \leq j \leq m+1, e_{j} \preceq e_{p}^{\prime \prime}$ for all $\varphi(j-1)<p \leq \varphi(j)$, and

4. for each $m+1 \leq j \leq m+m^{\prime}+1, e_{j}^{\prime} \preceq e_{p}^{\prime \prime \prime}$ for all $\varphi^{\prime}(j-1-m)+n<p \leq \varphi^{\prime}(j-m)+n$.

And we obtain $\gamma * \gamma^{\prime} \preceq \delta * \delta^{\prime}$.

Lemma 3.15. The concatenation operation is associative, i.e.

$$
\left(\gamma_{1} * \gamma_{2}\right) * \gamma_{3}=\gamma_{1} *\left(\gamma_{2} * \gamma_{3}\right)
$$

for $\gamma_{1} \in Q(\mu)\left(c_{1}, c_{2}\right), \gamma_{2} \in Q(\mu)\left(c_{2}, c_{3}\right)$, and $\gamma_{3} \in Q(\mu)\left(c_{3}, c_{4}\right)$.

Proof. By definition.

The following relations are useful.

Lemma 3.16. For a pair of composable flow paths $\gamma=\left(e_{1}, u_{1}, \ldots, e_{m}, u_{m} ; \tau(\gamma)\right)$ and $\delta=$ $\left(e_{1}^{\prime}, u_{1}^{\prime}, \ldots, e_{n}^{\prime}, u_{n}^{\prime} ; \tau(\delta)\right)$, we have

1. $\delta \preceq \gamma * \delta$, when either

(a) $\ell(\delta) \geq 1$, or

(b) $\ell(\delta)=0$ and $\tau(\delta) \preceq e_{p}$ for all $1 \leq p \leq m$, and

2. $\gamma * \delta \preceq \gamma$, when either

(a) $\ell(\gamma) \geq 1$, or

(b) $\ell(\gamma)=0$ and $e_{1}^{\prime} \preceq \tau(\gamma)$.

Proof. Define $\varphi:\{0,1, \ldots, n+1\} \rightarrow\{0,1, \ldots, n+m+1\}$ by $\varphi(0)=0$ and $\varphi(i)=i+m$ for $i=1, \ldots, n+1$. Then the first three conditions for $\delta \preceq \gamma * \delta$ is obvious.

Suppose $n>0$. For each $1 \leq j \leq n+1, \varphi(j-1)<p \leq \varphi(j)$ implies that $p=m+j$. Thus the fourth condition is equivalent to $e_{j}^{\prime} \preceq e_{m+j}^{\prime \prime}=e_{j}^{\prime}$ for $j=1, \ldots, n$ where

$$
\gamma * \delta=\left(e_{1}^{\prime \prime}, u_{1}^{\prime \prime}, \ldots, e_{m+n}^{\prime \prime}, u_{m+n}^{\prime \prime} ; \tau(\delta)\right) .
$$

When $n=0$, the condition is $e_{1}^{\prime} \preceq e_{p}^{\prime \prime}$ for $\varphi(0)<p \leq \varphi(1)=m+1$. Or $\tau(\delta) \preceq e_{p}$ for $0<p \leq m+1$. Since $\tau(\delta)=e_{m+1}=\tau(\gamma)$ in this case, the essential condition is $\tau(\delta) \preceq e_{p}$ for $0<p \leq m$.

Let us show that the identity map $\{0,1, \ldots, m+1\} \rightarrow\{0,1, \ldots, m+1\}$ is an embedding function for $\gamma * \delta \preceq \gamma$ if $\ell(\gamma) \geq 1$. Again the first three conditions obviously hold. The fourth condition is equivalent to $e_{j} \preceq e_{j}$ for $1 \leq j \leq m$ and $e_{1}^{\prime} \preceq \tau(\gamma)$, when $\ell(\gamma) \geq 1$. Since $\gamma * \delta$ is defined, $e_{1}^{\prime} \preceq \tau(\gamma)$ holds. When $\ell(\gamma)=0$, the fourth condition is equivalent to $e_{1}^{\prime} \preceq \tau(\gamma)$. 
Example 3.17. Consider the function $f$ on the boundary $\partial\left[v_{0}, v_{1}, v_{2}\right]$ of a 2 -simplex defined by

$$
\begin{aligned}
f\left(\left[v_{0}\right]\right) & =0 \\
f\left(\left[v_{0}, v_{1}\right]\right) & =1 \\
f\left(\left[v_{0}, v_{2}\right]\right) & =2 \\
f\left(\left[v_{1}\right]\right) & =4 \\
f\left(\left[v_{2}\right]\right) & =5 \\
f\left(\left[v_{1}, v_{2}\right]\right) & =6 .
\end{aligned}
$$

Then this is a discrete Morse function whose partial matching is given by

$$
\begin{aligned}
& \mu_{f}\left(\left[v_{1}\right]\right)=\left[v_{0}, v_{1}\right] \\
& \mu_{f}\left(\left[v_{2}\right]\right)=\left[v_{0}, v_{2}\right] .
\end{aligned}
$$

It has two critical simplices $\left[v_{0}\right]$ and $\left[v_{1}, v_{2}\right]$. Here is a list of Forman paths:

$$
\begin{aligned}
\gamma_{1} \quad: \quad\left[v_{1}\right] \prec\left[v_{0}, v_{1}\right] \\
\gamma_{2}: \quad\left[v_{2}\right] \prec\left[v_{0}, v_{2}\right] .
\end{aligned}
$$

We regard each Forman path as a flow path by adding $\left[v_{0}\right]$ at the end:

$$
\begin{aligned}
\gamma_{1}: & {\left[v_{1}\right] \prec\left[v_{0}, v_{1}\right] \succ\left[v_{0}\right] } \\
\gamma_{2} & : \quad\left[v_{2}\right] \prec\left[v_{0}, v_{2}\right] \succ\left[v_{0}\right] .
\end{aligned}
$$

We have non-Forman flow paths as follows:

$$
\begin{aligned}
& \gamma_{0}:\left[v_{0}\right] \\
& \gamma_{01}: \quad\left[v_{0}, v_{1}\right] \preceq\left[v_{0}, v_{1}\right] \succ\left[v_{0}\right] \\
& \gamma_{02} \quad: \quad\left[v_{0}, v_{2}\right] \preceq\left[v_{0}, v_{2}\right] \succ\left[v_{0}\right] \\
& \gamma_{12}:\left[v_{1}, v_{2}\right]
\end{aligned}
$$

so that

$$
\operatorname{FP}(\mu)=\overline{\operatorname{FP}}(\mu)=\left\{\gamma_{0}, \gamma_{1}, \gamma_{2}, \gamma_{01}, \gamma_{02}, \gamma_{12}\right\}
$$

Note that

$$
\begin{aligned}
& \gamma_{01}=\gamma_{01} * \gamma_{0} \\
& \gamma_{02}=\gamma_{02} * \gamma_{0},
\end{aligned}
$$

which imply, by Lemma 3.16,

$$
\begin{aligned}
\gamma_{0} & \prec \gamma_{01} \\
\gamma_{0} & \prec \gamma_{02} .
\end{aligned}
$$

Note also that $\gamma_{0} \prec \gamma_{1}$ and $\gamma_{0} \prec \gamma_{2}$ do not hold even though $\gamma_{1}=\gamma_{1} * \gamma_{0}$ and $\gamma_{2}=\gamma_{2} * \gamma_{0}$.

Furthermore we also have

$$
\begin{aligned}
\gamma_{1} & \prec \gamma_{12} \\
\gamma_{2} & \prec \gamma_{12} \\
\gamma_{1} & \prec \gamma_{01} \\
\gamma_{2} & \prec \gamma_{02} .
\end{aligned}
$$


For example, an embedding function for $\gamma_{1} \prec \gamma_{01}$ is given by the identity map $\{0,1,2\} \rightarrow\{0,1,2\}$ and an embedding function for $\gamma_{1} \prec \gamma_{12}$ is given by the identity map $\{0,1\} \rightarrow\{0,1\}$.

Thus the Hasse diagram is given by

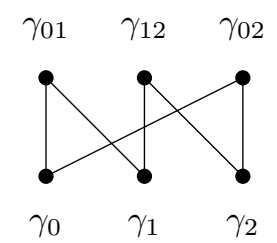

which implies that $B \mathrm{FP}(\mu)$ is the boundary of a hexagon and can be identified with the barycentric subdivision of $X=\partial\left[v_{0}, v_{1}, v_{2}\right]$.

Here is a definition of the flow category $C(\mu)$.

Definition 3.18 (Flow category). Define a category $C(\mu)$ as follows. The set of objects is given by

$$
C(\mu)_{0}=\operatorname{Cr}(\mu) .
$$

The set of morphisms $C(\mu)\left(c, c^{\prime}\right)$ from a critical cell $c$ to another $c^{\prime}$ is given

$$
C(\mu)\left(c, c^{\prime}\right)= \begin{cases}Q(\mu)\left(c^{\prime}, c\right), & c \neq c^{\prime} \\ \left\{1_{c}\right\}, & c=c .\end{cases}
$$

The composition $\circ$ is defined by the concatenation $*$

$$
\circ: C(\mu)\left(c_{2}, c_{3}\right) \times C(\mu)\left(c_{1}, c_{2}\right)=Q(\mu)\left(c_{3}, c_{2}\right) \times Q(\mu)\left(c_{2}, c_{1}\right) \stackrel{*}{\longrightarrow} Q(\mu)\left(c_{3}, c_{1}\right)=C(\mu)\left(c_{1}, c_{3}\right) .
$$

This category $C(\mu)$ is called the flow category of $\mu$. When $\mu=\mu_{f}$ for a discrete Morse function, we also use the notation $C(f)$.

As a corollary to Lemma 3.14, we see that $C(\mu)$ is a poset category. In particular it is a 2 -category and we may apply any of the classifying space functors reviewed in $\S$ A.2, i.e. $B^{2}, B^{c l}$, and $B^{n c l}$. By Theorem A.35, these constructions are weakly homotopy equivalent to each other.

Example 3.19. Consider the flow paths in Example 3.17. We have

$$
C(\mu)\left(\left[v_{0}\right],\left[v_{1}, v_{2}\right]\right)=Q(\mu)\left(\left[v_{1}, v_{2}\right],\left[v_{0}\right]\right)=\left\{\gamma_{1}, \gamma_{2}\right\}
$$

with trivial order relation. Thus $C(\mu)$ is a 1-category and we have a homeomorphism

$$
B C(\mu) \cong \partial\left[v_{0}, v_{1}, v_{2}\right] .
$$

We would like to show that this example generalizes, i.e. the classifying space of the flow category $C(\mu)$ is always homotopy equivalent to $X$. It is not easy, however, to relate these spaces directly. We need intermediate categories and a zigzag of functors

$$
F\left(\operatorname{Sd}_{f}(X)\right) \longleftarrow \overline{\mathrm{FP}}(\mu) \stackrel{\tau}{\longrightarrow} \bar{C}(\mu) \stackrel{r}{\longleftarrow} C(\mu) .
$$

$\operatorname{Sd}_{f}(X)$ is a subdivision of $X$ which will be defined in $\S 3.4 . \overline{\mathrm{FP}}(\mu)$ is the poset of reduced flow paths defined in Definition 2.17 and 2.20. The rest of this section is devoted to the construction of the reduced flow category $\bar{C}(\mu)$ and a functor $r: C(\mu) \rightarrow \bar{C}(\mu)$ which induces a homotopy equivalence of classifying spaces. 
Definition 3.20. Define a poset quiver $\bar{C}(\mu)$ by

$$
\begin{aligned}
\bar{C}(\mu)_{0} & =\operatorname{Cr}(\mu) \\
\bar{C}(\mu)\left(c, c^{\prime}\right) & =\bar{Q}(\mu)\left(c^{\prime}, c\right) .
\end{aligned}
$$

Namely $\bar{C}(\mu)$ is a subquiver of $C(\mu)$ consisting of reduced flow paths. Unfortunately $\bar{C}(\mu)$ is not closed under the composition in $C(\mu)$. We need to take a reduction after the concatenation.

Definition 3.21. For $\gamma \in \bar{C}(\mu)\left(c_{1}, c_{2}\right)$ and $\delta \in \bar{C}(\mu)\left(c_{2}, c_{3}\right)$, define $\delta \circ \gamma \in \bar{C}(\mu)\left(c_{1}, c_{3}\right)$ by

$$
\delta \circ \gamma=r(\gamma * \delta) .
$$

Note that

$$
\circ: \bar{C}(\mu)\left(c_{2}, c_{3}\right) \times \bar{C}(\mu)\left(c_{1}, c_{2}\right) \longrightarrow \bar{C}(\mu)\left(c_{1}, c_{3}\right)
$$

is a poset map as the composition of poset maps $r$ and $*$.

Proposition 3.22. The following diagram is commutative

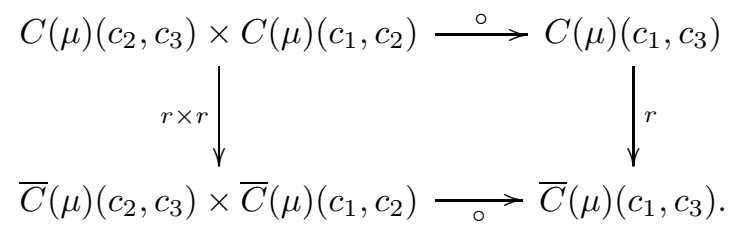

Proof. By Lemma 2.29, $r(\delta) \preceq \delta$ and $r(\gamma) \preceq \gamma$. Since $r$ and $*$ are poset maps by Lemma 3.14 and Lemma 2.29, we have

$$
r(\gamma) \circ r(\delta)=r(r(\delta) * r(\gamma)) \preceq r(\delta * \gamma)
$$

for $(\gamma, \delta) \in C(\mu)\left(c_{2}, c_{3}\right) \times C(\mu)\left(c_{1}, c_{2}\right)$. On the other hand, $r(\delta * \gamma)$ is obtained from $\delta * \gamma$ by removing cells having indices in reducible intervals of $\delta * \gamma$. Note, however, it can be also obtained by removing cells indexed by reducible intervals in $\delta$ and $\gamma$, and then removing reducible intervals of the resulting path. In particular, $r(\delta * \gamma)$ is a subpath of $r(\delta) * r(\gamma)$. Since $r$ is a poset map and a retraction, we have

$$
r(\delta * \gamma)=r(r(\delta * \gamma)) \preceq r(r(\delta) * r(\gamma))=r(\gamma) \circ r(\delta) .
$$

Corollary 3.23. The following diagram is commutative

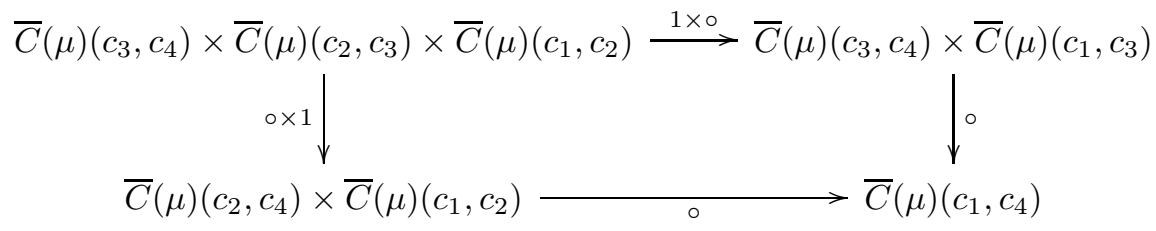

for any critical cells $c_{1}, c_{2}, c_{3}$ and $c_{4}$.

Proof. Since $r$ is surjective by Lemma 2.29 and is compatible with $\circ$ by Proposition 3.22, the result follows from Lemma 3.15 . 
Definition 3.24 (Reduced flow category). The poset category obtained from the quiver $\bar{Q}(\mu)$ by using the composition in Definition 3.21 is called the reduced flow category $\bar{C}(\mu)$.

Proposition 3.22 implies that the reduction $r$ is a functor of poset categories from $C(\mu)$ to $\bar{C}(\mu)$. The following is an immediate but important consequence of Proposition 2.30.

Theorem 3.25. The reduction $r: C(\mu) \rightarrow \bar{C}(\mu)$ induces a homotopy equivalence

$$
B^{2} r: B^{2} C(\mu) \longrightarrow B^{2} \bar{C}(\mu) .
$$

Proof. By Proposition 2.30, the reduction $r$ is a descending closure operator, which implies that it induces a deformation retraction on each classifying space of morphisms

$$
B r\left(c, c^{\prime}\right): B C(\mu)\left(c, c^{\prime}\right) \stackrel{\simeq}{\longrightarrow} B \bar{C}(\mu)\left(c, c^{\prime}\right)
$$

by Corollary A.22. By applying Proposition A.31, we see that $B^{2} r: B^{2} C(\mu) \rightarrow B^{2} \bar{C}(\mu)$ is a homotopy equivalence.

\subsection{The Collapsing Functor}

Theorem 3.25 says that the right most functor in (3.4) induces a homotopy equivalence between classifying spaces. The next step is to define a functor $\tau: \overline{\mathrm{FP}}(\mu) \rightarrow \bar{C}(\mu)$ and show that it induces a homotopy equivalence between classifying spaces.

Notice that $\bar{C}(\mu)$ is a poset-category, hence a 2-category. On the other hand, $\overline{\mathrm{FP}}(\mu)$ is a poset regarded as a small category. We should regard $\overline{\mathrm{FP}}(\mu)$ as a 2-category whose 2-morphisms are identities and try to construct a 2-functor $\tau: \overline{\mathrm{FP}}(\mu) \rightarrow \bar{C}(\mu)$. On objects it is given by the target map

$$
\tau: \mathrm{FP}(\mu) \longrightarrow \operatorname{Cr}(\mu)=C(\mu)_{0} .
$$

The restriction to $\overline{\mathrm{FP}}(\mu)$ is denoted by $\bar{\tau}$.

We would like to extend these maps to 2 -functors by regarding the posets $\operatorname{FP}(\mu)$ and $\overline{\mathrm{FP}}(\mu)$ as small categories. Unfortunately this is too much to expect. The best we can do is the following.

Proposition 3.26. The map $\tau$ can be extended to a normal colax functor ${ }^{5} \tau: \operatorname{FP}(\mu) \rightarrow C(\mu)$. Furthermore it induces a normal colax functor $\bar{\tau}: \overline{\mathrm{FP}}(\mu) \rightarrow \bar{C}(\mu)$.

Proof. For a morphism $\gamma \preceq \gamma^{\prime}$ in $\operatorname{FP}(\mu)$, let $\varphi:\{0, \ldots, k\} \rightarrow\{0, \ldots, n+1\}$ be the embedding function. Define a flow path $\tau\left(\gamma \preceq \gamma^{\prime}\right)$ to be the subsequence of $\gamma=\left(e_{1}, u_{1}, \ldots, e_{n}, u_{n} ; \tau(\gamma)\right)$ starting at $e_{k}$. Note that we have $e_{k} \preceq \tau\left(\gamma^{\prime}\right)$ by Remark 2.21 and we have

$$
\tau\left(\gamma \preceq \gamma^{\prime}\right)=\left(e_{k}, u_{k}, \ldots, e_{n}, u_{n} ; \tau(\gamma)\right) \in Q(\mu)\left(\tau\left(\gamma^{\prime}\right), \tau(\gamma)\right)=C(\mu)\left(\tau(\gamma), \tau\left(\gamma^{\prime}\right)\right) .
$$

Then $\tau$ sends the identity $\gamma \preceq \gamma$ to $1_{\tau(\gamma)}$ by definition.

For a sequence $\gamma_{1} \preceq \gamma_{2} \preceq \gamma_{3}$ in $\operatorname{FP}(\mu)$, let us show that

$$
\tau\left(\gamma_{1} \preceq \gamma_{3}\right) \preceq \tau\left(\gamma_{2} \preceq \gamma_{3}\right) \circ \tau\left(\gamma_{1} \preceq \gamma_{2}\right)
$$

in $C(\mu)\left(\tau\left(\gamma_{1}\right), \tau\left(\gamma_{3}\right)\right)$. Let

$$
\begin{aligned}
& \varphi_{1}:\left\{0, \ldots, k_{1}\right\} \longrightarrow\left\{0, \ldots, n_{2}+1\right\} \\
& \varphi_{2}:\left\{0, \ldots, k_{2}\right\} \longrightarrow\left\{0, \ldots, n_{3}+1\right\}
\end{aligned}
$$

\footnotetext{
${ }^{5}$ See Definition 3.7.
} 
be embedding functions for $\gamma_{1} \preceq \gamma_{2}$ and $\gamma_{2} \preceq \gamma_{3}$, respectively. By definition, the flow path $\tau\left(\gamma_{1} \preceq\right.$ $\left.\gamma_{2}\right)$ is the subsequence of $\gamma_{1}=\left(e_{1}, u_{1}, \ldots, e_{n_{1}}, u_{n_{1}} ; \tau\left(\gamma_{1}\right)\right)$ starting at the cell $e_{k_{1}}$ and $\tau\left(\gamma_{2} \preceq \gamma_{3}\right)$ is the subsequence of $\gamma_{2}=\left(e_{1}^{\prime}, u_{1}^{\prime}, \ldots, e_{n_{2}}^{\prime}, u_{n_{2}}^{\prime} ; \tau\left(\gamma_{2}\right)\right)$ starting at the cell $e_{k_{2}}^{\prime}$. According to the proof of Proposition 2.28, on the other hand, the embedding function $\psi:\left\{0, \ldots, k_{3}\right\} \rightarrow$ $\left\{0, \ldots, n_{3}+1\right\}$ for $\gamma_{1} \preceq \gamma_{3}$ is given by

$$
\psi(i)= \begin{cases}\left(\varphi_{2} \circ \varphi_{1}\right)(i), & i<k_{3} \\ \varphi_{2}\left(k_{2}\right)=n_{3}+1, & i=k_{3}\end{cases}
$$

where $k_{3}$ is the number with $\varphi_{1}\left(k_{3}-1\right)<k_{2} \leq \varphi_{1}\left(k_{3}\right)$. Thus we have

$$
\begin{aligned}
\tau\left(\gamma_{2} \preceq \gamma_{3}\right) * \tau\left(\gamma_{1} \preceq \gamma_{2}\right) & =\left(e_{k_{2}}^{\prime}, u_{k_{2}}^{\prime}, \ldots, e_{n_{2}}^{\prime}, u_{n_{2}}^{\prime}, e_{k_{1}}, u_{k_{1}}, \ldots, e_{n_{1}}, u_{n_{1}}, ; \tau\left(\gamma_{1}\right)\right) \\
\tau\left(\gamma_{1} \preceq \gamma_{3}\right) & =\left(e_{k_{3}}, u_{k_{3}}, \ldots, e_{n_{1}}, u_{n_{1}} ; \tau\left(\gamma_{1}\right)\right) .
\end{aligned}
$$

Let us rename cells in these flow paths as

$$
\begin{aligned}
\tilde{e}_{j} & =e_{j+k_{3}-1} \\
\tilde{u}_{j} & =u_{j+k_{3}-1}
\end{aligned}
$$

in $\tau\left(\gamma_{1} \preceq \gamma_{3}\right)$ and

$$
\begin{aligned}
\tilde{e}_{j}^{\prime} & = \begin{cases}e_{j+k_{2}-1}^{\prime}, & 1 \leq j \leq n_{2}-k_{2}+1 \\
e_{j+k_{1}+k_{2}-n_{2}-2}, & n_{2}-k_{2}+2 \leq j \leq n_{1}+n_{2}-k_{1}-k_{2}+2\end{cases} \\
\tilde{u}_{j}^{\prime} & = \begin{cases}u_{j+k_{2}-1}^{\prime}, & 1 \leq j \leq n_{2}-k_{2}+1 \\
u_{j+k_{1}+k_{2}-n_{2}-2}, & n_{2}-k_{2}+2 \leq j \leq n_{1}+n_{2}-k_{1}-k_{2}+2\end{cases}
\end{aligned}
$$

in $\tau\left(\gamma_{2} \preceq \gamma_{3}\right) * \tau\left(\gamma_{1} \preceq \gamma_{2}\right)$ so that we have

$$
\begin{aligned}
\tau\left(\gamma_{1} \preceq \gamma_{3}\right) & =\left(\tilde{e}_{1}, \tilde{u}_{1}, \ldots, \tilde{e}_{n_{1}-k_{3}+1}, \tilde{u}_{n_{1}-k_{3}+1} ; \tau\left(\gamma_{1}\right)\right) \\
\tau\left(\gamma_{2} \preceq \gamma_{3}\right) * \tau\left(\gamma_{1} \preceq \gamma_{2}\right) & =\left(\tilde{e}_{1}^{\prime}, \tilde{u}_{1}^{\prime}, \ldots, \tilde{e}_{n_{1}+n_{2}-k_{1}-k_{2}+2}^{\prime}, \tilde{u}_{n_{1}+n_{2}-k_{1}-k_{2}+2}^{\prime} ; \tau\left(\gamma_{1}\right)\right) .
\end{aligned}
$$

With this notation, what we need is a map

$$
\zeta:\left\{0,1, \ldots, n_{1}-k_{3}+2\right\} \longrightarrow\left\{0,1, \ldots, n_{1}+n_{2}-k_{1}-k_{2}+3\right\}
$$

satisfying the following conditions:

1. $\zeta(0)=0$,

2. $\tilde{u}_{j}=\tilde{u}_{\zeta(j)}^{\prime}$ for $1 \leq j<n_{1}-k_{3}+2$,

3. $\zeta\left(n_{1}-k_{3}+2\right)=n_{1}+n_{2}-k_{1}-k_{2}+3$,

4. $\tilde{e}_{j} \preceq \tilde{e}_{p}^{\prime}$ for $\zeta(j-1)<p \leq \zeta(j)$ and $1 \leq j \leq n_{1}-k_{3}+2$.

Define $\zeta$ by

$$
\zeta(j)= \begin{cases}0, & j=0 \\ \varphi_{1}\left(j+k_{3}-1\right)-k_{2}+1, & 1 \leq j \leq k_{1}-k_{3} \\ j+n_{2}-k_{1}-k_{2}+k_{3}+1, & k_{1}-k_{3}+1 \leq j \leq n_{1}-k_{3}+2\end{cases}
$$


Let us verify that $\zeta$ is an embedding function for

$$
\tau\left(\gamma_{1} \preceq \gamma_{3}\right) \preceq \tau\left(\gamma_{2} \preceq \gamma_{3}\right) * \tau\left(\gamma_{1} \preceq \gamma_{2}\right) .
$$

The first and the third conditions are obvious.

For the second condition, suppose $1 \leq j \leq k_{1}-k_{3}$. Then

$$
\begin{aligned}
\tilde{u}_{j} & =u_{j+k_{3}-1} \\
\tilde{u}_{\zeta(j)}^{\prime} & =u_{\zeta(j)+k_{2}-1}^{\prime \prime} \\
& =u_{\varphi_{1}\left(j+k_{3}-1\right)}^{\prime} .
\end{aligned}
$$

By the second condition for the embedding function $\varphi_{1}$, these cells coincide. When $k_{1}-k_{3}+1 \leq$ $j \leq n_{1}-k_{3}+2$, we have

$$
n_{2}-k_{2}+2 \leq \zeta(j) \leq n_{1}+n_{2}-k_{1}-k_{2}+3 .
$$

and thus

$$
\begin{aligned}
\tilde{u}_{\zeta(j)}^{\prime} & =u_{\zeta(j)+k_{1}+k_{2}-n_{2}-2} \\
& =u_{j+n_{2}-k_{1}-k_{2}+k_{3}+1+k_{1}+k_{2}-n_{2}-2} \\
& =u_{j+k_{3}-1} .
\end{aligned}
$$

On the other hand, $\tilde{u}_{j}=u_{j+k_{3}-1}$ by definition and we have $\tilde{u}_{\zeta(j)}^{\prime}=\tilde{u}_{j}$.

For the fourth condition, suppose $1 \leq j \leq k_{1}-k_{3}$ and $\zeta(j-1)<p \leq \zeta(j)$. In this case $\zeta(j-1)=\varphi_{1}\left(j+k_{3}-2\right)-k_{2}+1$ and $\zeta(j)=\varphi_{1}\left(j+k_{3}-1\right)-k_{2}+1$, which implies that

$$
\varphi_{1}\left(j+k_{3}-2\right)<p+k_{2}-1 \leq \varphi_{1}\left(j+k_{3}-1\right) .
$$

The fourth condition for $\varphi_{1}$ implies that

$$
\tilde{e}_{j}=e_{j+k_{3}-1} \preceq e_{p+k_{2}-1}^{\prime}=\tilde{e}_{p}^{\prime} .
$$

When $k_{1}-k_{3}+1 \leq j \leq n_{1}-k_{3}+2$, the condition $\zeta(j-1)<p \leq \zeta(j)$ is equivalent to $p=j+n_{2}-k_{1}-k_{2}+k_{3}+1$, in which case

$$
\begin{aligned}
\tilde{e}_{p}^{\prime} & =e_{p+k_{1}+k_{2}-n_{2}-2} \\
& =e_{j+k_{3}-1}
\end{aligned}
$$

which coincides with $\tilde{e}_{j}=e_{j+k_{3}-1}$. And we obtain an embedding function $\zeta$ for (3.5).

Suppose $\gamma \preceq \delta$ in $\overline{\mathrm{FP}}(\mu)$. Since $\tau(\gamma \preceq \delta)$ is defined as a subsequence of $\gamma, \tau(\gamma \preceq \delta)$ belongs to $\bar{C}(\mu)(\tau(\gamma), \tau(\delta))$. For a sequence $\gamma_{1} \preceq \gamma_{2} \preceq \gamma_{3}$ in $\overline{\mathrm{FP}}(\mu)$, we have $(3.5)$ in $Q(\mu)\left(\tau\left(\gamma_{3}\right), \tau\left(\gamma_{1}\right)\right)$. Since the reduction $r$ is a poset map and $\tau\left(\gamma_{1} \preceq \gamma_{3}\right)$ is reduced, we obtain

$$
\left.\tau\left(\gamma_{1} \preceq \gamma_{3}\right)=r\left(\tau\left(\gamma_{1} \preceq \gamma_{3}\right)\right) \preceq r\left(\tau\left(\gamma_{2} \preceq \gamma_{3}\right) \circ \tau\left(\gamma_{1} \preceq \gamma_{2}\right)\right)=\tau\left(\gamma_{2} \preceq \gamma_{3}\right) \circ \tau\left(\gamma_{1} \preceq \gamma_{2}\right)\right) .
$$

in $\bar{C}(\mu)\left(\tau\left(\gamma_{1}\right), \tau\left(\gamma_{3}\right)\right)$. And we obtain a normal colax functor

$$
\bar{\tau}: \overline{\mathrm{FP}}(\mu) \longrightarrow \bar{C}(\mu) .
$$


Example 3.27. Consider the discrete Morse function in Example 3.17. Its flow category is described in Example 3.19.

The collapsing functor $\tau$ is given, on objects, by

$$
\begin{aligned}
\tau\left(\gamma_{0}\right) & =\left[v_{0}\right] \\
\tau\left(\gamma_{1}\right) & =\left[v_{0}\right] \\
\tau\left(\gamma_{2}\right) & =\left[v_{0}\right] \\
\tau\left(\gamma_{01}\right) & =\left[v_{0}\right] \\
\tau\left(\gamma_{02}\right) & =\left[v_{0}\right] \\
\tau\left(\gamma_{12}\right) & =\left[v_{1}, v_{2}\right] .
\end{aligned}
$$

We have six nontrivial order relations in $\mathrm{FP}(\mu)$, for which the collapsing functor is defined by

$$
\begin{aligned}
\tau\left(\gamma_{0} \prec \gamma_{01}\right) & =\left(\left[v_{0}\right]\right)=\gamma_{0}=1_{\left[v_{0}\right]} \\
\tau\left(\gamma_{0} \prec \gamma_{02}\right) & =\left(\left[v_{0}\right]\right)=\gamma_{0}=1_{\left[v_{0}\right]} \\
\tau\left(\gamma_{1} \prec \gamma_{01}\right) & =\left(\left[v_{0}\right]\right)=\gamma_{0}=1_{\left[v_{0}\right]} \\
\tau\left(\gamma_{2} \prec \gamma_{02}\right) & =\left(\left[v_{0}\right]\right)=\gamma_{0}=1_{\left[v_{0}\right]} \\
\tau\left(\gamma_{1} \prec \gamma_{12}\right) & =\gamma_{1} \\
\tau\left(\gamma_{2} \prec \gamma_{12}\right) & =\gamma_{2} .
\end{aligned}
$$

By Lemma A.33, $\tau$ and $\bar{\tau}$ induce maps between normal colax classifying spaces

$$
\begin{array}{lll}
B^{n c l} \tau & : \quad B^{n c l} \operatorname{FP}(\mu)=B \mathrm{FP}(\mu) \longrightarrow B^{n c l} C(\mu) \\
B^{n c l} \bar{\tau} & : \quad B^{n c l} \overline{\mathrm{FP}}(\mu)=B \overline{\mathrm{FP}}(\mu) \longrightarrow B^{n c l} \bar{C}(\mu) .
\end{array}
$$

Let us show that both $B^{n c l} \tau$ and $B^{n c l} \bar{\tau}$ are homotopy equivalences. One of the most convenient tools for showing a functor to be a homotopy equivalence is Quillen's Theorem A, which says that a functor between small categories induces a homotopy equivalence between classifying spaces if "(homotopy) fibers" are contractible. There are variations, depending on the three choices for (homotopy) fibers; $\tau \downarrow c, c \downarrow \tau$, and $\tau^{-1}(c)$. For functors between 2-categories, analogous theorems have been proved. Here we use Corollary A.51 by showing that $\tau$ is prefibered and each fiber $\tau^{-1}(c)$ is contractible.

Let us first find explicit descriptions of homotopy fibers in order to show that $\tau$ is prefibered. Note that both left and right homotopy fibers are posets by Corollary A.47.

Lemma 3.28. For a critical cell $c$, the posets $\tau \downarrow c$ and $c \downarrow \tau$ are given by

$$
\begin{aligned}
& \tau \downarrow c=\left\{(\gamma, \delta) \in \operatorname{FP}(\mu)^{2} \mid \sigma(\delta)=c, \tau(\delta)=\tau(\gamma)\right\} \\
& c \downarrow \tau=\left\{(\delta, \gamma) \in \operatorname{FP}(\mu)^{2} \mid \tau(\gamma)=\sigma(\delta), \tau(\delta)=c\right\},
\end{aligned}
$$

respectively. The partial orders $\preceq_{c}$ and $\preceq^{c}$ on $\tau \downarrow c$ and $c \downarrow \tau$ are, respectively, given by

$$
\begin{aligned}
& (\gamma, \delta) \preceq_{c}\left(\gamma^{\prime}, \delta^{\prime}\right) \Longleftrightarrow \gamma \preceq \gamma^{\prime} \text { and } \delta^{\prime} * \tau\left(\gamma \preceq \gamma^{\prime}\right) \preceq \delta . \\
& (\delta, \gamma) \preceq^{c}\left(\delta^{\prime}, \gamma^{\prime}\right) \Longleftrightarrow \gamma \preceq \gamma^{\prime} \text { and } \tau\left(\gamma \preceq \gamma^{\prime}\right) * \delta \preceq \delta^{\prime} .
\end{aligned}
$$

Proof. Let us consider the case of the left homotopy fiber. We have

$$
\begin{aligned}
(\tau \downarrow c)_{0} & =\left\{(\gamma, \delta) \in \operatorname{FP}(\mu) \times C(\mu)_{1} \mid \delta \in C(\mu)(\tau(\gamma), c)\right\} \\
& =\left\{(\gamma, \delta) \in \operatorname{FP}(\mu)^{2} \mid \delta \in Q(\mu)(c, \tau(\gamma))\right\} \\
& =\left\{(\gamma, \delta) \in \operatorname{FP}(\mu)^{2} \mid \sigma(\delta)=c, \tau(\delta)=\tau(\gamma)\right\} .
\end{aligned}
$$


By Corollary A.47, the partial order $\preceq_{c}$ on $\tau \downarrow c$ is given by

$$
(\gamma, \delta) \preceq_{c}\left(\gamma^{\prime}, \delta^{\prime}\right) \Longleftrightarrow \gamma \preceq \gamma^{\prime} \text { and } \delta^{\prime} * \tau\left(\gamma \preceq \gamma^{\prime}\right) \preceq \delta .
$$

The right homotopy fiber is given by

$$
\begin{aligned}
(c \downarrow \tau)_{0} & =\left\{(\delta, \gamma) \in C(\mu)_{1} \times \operatorname{FP}(\mu) \mid \delta \in C(\mu)(c, \tau(\gamma))\right\} \\
& =\left\{(\delta, \gamma) \in \operatorname{FP}(\mu)^{2} \mid \delta \in Q(\mu)(\tau(\gamma), c)\right\} \\
& =\left\{(\delta, \gamma) \in \operatorname{FP}(\mu)^{2} \mid \tau(\gamma)=\sigma(\delta), \tau(\delta)=c\right\}
\end{aligned}
$$

The partial order on this poset set is given by

$$
(\delta, \gamma) \preceq^{c}\left(\delta^{\prime}, \gamma^{\prime}\right) \Longleftrightarrow \gamma \preceq \gamma^{\prime} \text { and } \tau\left(\gamma \preceq \gamma^{\prime}\right) * \delta \preceq \delta^{\prime} .
$$

Proposition 3.29. For each $c \in \operatorname{Cr}(\mu)$, define maps $i_{c}: \tau^{-1}(c) \rightarrow c \downarrow \tau$ and $s_{c}: c \downarrow \tau \rightarrow \tau^{-1}(c)$ by $i_{c}(\gamma)=\left(1_{c}, \gamma\right)$ and $s_{c}(\delta, \gamma)=\gamma * \delta$, respectively. Then the composition

$$
\rho_{c}: c \downarrow \tau \stackrel{s_{c}}{\longrightarrow} \tau^{-1}(c) \stackrel{i_{c}}{\longrightarrow} c \downarrow \tau
$$

is a descending closure operator.

Proof. Obviously $\rho_{c} \circ \rho_{c}=\rho_{c}$. Since $\gamma * \delta \preceq \gamma$ by Lemma 3.16 and $\tau(\gamma * \delta \preceq \gamma)=\delta$, we have

$$
\rho_{c}(\delta, \gamma)=\left(1_{\tau(\gamma * \delta)}, \gamma * \delta\right) \preceq^{c}(\delta, \gamma)
$$

in $c \downarrow \tau$.

This implies that $\rho$ is the counit for the adjunction $i \dashv s$ and we obtain the following corollary.

Corollary 3.30. The collapsing functor $\tau$ is prefibered.

Now we are ready to prove the following theorem.

Theorem 3.31. For an acyclic partial matching $\mu$ on a finite regular $C W$ complex, the collapsing functor $\tau$ induces a homotopy equivalence

$$
B^{n c l} \tau: B \mathrm{FP}(\mu) \longrightarrow B^{n c l} C(\mu)
$$

between classifying spaces.

Proof. By Corollary 3.30 and Corollary A.51, it suffices to show that $B \tau^{-1}(c)$ is contractible for each $c \in \operatorname{Cr}(\mu)$.

Recall from Proposition 2.10 that we may choose a faithful and $\mathbb{Z}$-valued discrete Morse function $f$ whose associated partial matching is $\mu$. In particular, it is injective. For each nonnegative integer $\ell$, define

$$
\tau^{-1}(c)_{\ell}=(f \circ \iota)^{-1}(\ell) \cap \tau^{-1}(c) .
$$

Define a filtration on $\tau^{-1}(c)$ by

$$
F_{\ell} \tau^{-1}(c)=\bigcup_{i=0}^{\ell} \tau^{-1}(c)_{i} .
$$

We are going to show that $B F_{\ell} \tau^{-1}(c)$ deformation-retracts onto $B F_{\ell-1} \tau^{-1}(c)$ for all $\ell \geq 1$.

By the injectivity of $f, f^{-1}(\ell)$ contains at most one element. There are three cases; 
1. $f^{-1}(\ell)=\emptyset$

2. $f^{-1}(\ell)=\{d\}$ with $d \in D\left(\mu_{f}\right)$

3. $f^{-1}(\ell)=\left\{\mu_{f}(d)\right\}$ with $d \in D\left(\mu_{f}\right)$.

In the first case, $F_{\ell} \tau^{-1}(c)=F_{\ell-1} \tau^{-1}(c)$ and there is nothing to prove.

Suppose $\tau^{-1}(c)_{\ell}=\{d\}$ with $d \in D\left(\mu_{f}\right)$. Define a map

$$
m_{\ell}: F_{\ell} \tau^{-1}(c) \rightarrow F_{\ell} \tau^{-1}(c)
$$

by

$$
m_{\ell}(\gamma)= \begin{cases}\left(\mu_{f}(d), \mu_{f}(d), e_{2}, u_{2}, \ldots, e_{n}, u_{n} ; c\right), & \text { if } \gamma=\left(d, \mu_{f}(d), e_{2}, u_{2}, \ldots, e_{n}, u_{n} ; c\right), \\ \gamma, & \text { otherwise. }\end{cases}
$$

Since $f\left(\mu_{f}(d)\right) \leq f(d), m_{\ell}(\gamma)$ belongs to $F_{\ell} \tau^{-1}(c)$. Let us verify that this is an ascending closure operator on $F_{\ell} \tau^{-1}(c)$. The embedding function for $\gamma \preceq m_{\ell}(\gamma)$ is given by the identity map. We have $m_{\ell} \circ m_{\ell}=m_{\ell}$ by definition. It remains to show that this is a poset map.

Suppose $\gamma \preceq \gamma^{\prime}$ with embedding function $\varphi$. In particular, we have $\iota(\gamma) \preceq \iota\left(\gamma^{\prime}\right)$. Since $f$ is faithful, we have $f(\iota(\gamma)) \leq f\left(\iota\left(\gamma^{\prime}\right)\right)$.

When $\iota(\gamma) \neq d$, we have

$$
m_{\ell}(\gamma)=\gamma \preceq \gamma^{\prime} \preceq m_{\ell}\left(\gamma^{\prime}\right) .
$$

When $\iota(\gamma)=d$ the injectivity of $f$ implies that $\left.\iota\left(\gamma^{\prime}\right)\right)=\iota(\gamma)=d$, for $\ell=f(\iota(\gamma)) \leq f\left(\iota\left(\gamma^{\prime}\right)\right) \leq \ell$. Thus the same embedding function $\varphi$ serves as an embedding function for $m_{\ell}(\gamma) \preceq m_{\ell}\left(\gamma^{\prime}\right)$. And we have an ascending closure operator $m_{\ell}$ whose image is $F_{\ell-1} \tau^{-1}(c)$. By Corollary A.22, $B F_{\ell-1} \tau^{-1}(c)$ is a deformation retract of $B F_{\ell} \tau^{-1}(c)$.

Let us consider the third case $\tau^{-1}(c)_{\ell}=\left\{\mu_{f}(d)\right\}$. Define

$$
b_{\ell}: F_{\ell} \tau^{-1}(c) \longrightarrow F_{\ell} \tau^{-1}(c)
$$

by

$$
b_{\ell}(\gamma)= \begin{cases}\left(e_{2}, u_{2}, \ldots, e_{n}, u_{n} ; c\right), & \text { if } \iota(\gamma)=\mu_{f}(d) \\ \gamma, & \text { otherwise }\end{cases}
$$

for $\gamma=\left(e_{1}, u_{1}, e_{2}, u_{2}, \ldots, e_{n}, u_{n} ; c\right)$. As we have seen in Remark 2.12, the faithfulness of $f$ implies that

$$
\ell=f\left(\mu_{f}(d)\right) \geq f\left(\mu_{f}(d)\right)>f\left(e_{2}\right) \geq f\left(u_{2}\right)>\cdots>f\left(e_{n}\right) \geq f\left(u_{n}\right)>f(\tau(\gamma))
$$

and thus $b_{\ell}(\gamma) \in F_{\ell} \tau^{-1}(c)$.

Let us show that this is a descending closure operator. For a flow path $\gamma$ with $\iota(\gamma)=\mu_{f}(d)$, consider the map $d^{1}:\{0, \ldots, \ell\} \rightarrow\{0, \ldots, \ell+1\}$ given by

$$
d^{1}(i)= \begin{cases}0, & i=0 \\ i+1, & i \geq 2 .\end{cases}
$$

This serves as an embedding function for $b_{\ell}(\gamma) \preceq \gamma$, since $e_{2} \preceq \mu\left(d_{1}\right)$. The map $b_{\ell}$ is idempotent by definition. It remains to show that $b_{\ell}$ is a poset map. Suppose $\gamma \preceq \gamma^{\prime}$ with embedding function $\varphi:\{0, \ldots, \ell(\gamma)+1\} \rightarrow\left\{0, \ldots, \ell\left(\gamma^{\prime}\right)+1\right\}$. As is the case of $\tau^{-1}(c)_{\ell}=\{d\}, \gamma \preceq \gamma^{\prime}$ implies that $f(\iota(\gamma)) \leq f\left(\iota\left(\gamma^{\prime}\right)\right)$ and we have the following three cases.

1. $f(\iota(\gamma)) \leq f\left(\iota\left(\gamma^{\prime}\right)\right)<\ell$ 


\section{2. $f(\iota(\gamma))<f\left(\iota\left(\gamma^{\prime}\right)\right)=\ell$ \\ 3. $f(\iota(\gamma))=f\left(\iota\left(\gamma^{\prime}\right)\right)=\ell$}

When $f\left(\iota\left(\gamma^{\prime}\right)\right)<\ell$, we have $b_{\ell}(\gamma)=\gamma \preceq \gamma^{\prime}=b_{\ell}(\gamma)$. When $f(\iota(\gamma))=f\left(\iota\left(\gamma^{\prime}\right)\right)=\ell$, the restriction of the $\varphi$ to $\{0,2, \ldots, \ell(\gamma)+1\} \rightarrow\left\{0,2, \ldots, \ell\left(\gamma^{\prime}\right)+1\right\}$ is an embedding function for $b_{\ell}(\gamma) \preceq b_{\ell}\left(\gamma^{\prime}\right)$. Suppose $f(\iota(\gamma))<f\left(\iota\left(\gamma^{\prime}\right)\right)=\ell$. We need to show that $\gamma \preceq b_{\ell}\left(\gamma^{\prime}\right)$. Let $\gamma=$ $\left(e_{1}, u_{1}, e_{2}, u_{2}, \ldots, e_{n}, u_{n} ; c\right)$. Since $f(\iota(\gamma))<\ell$, the injectivity of $f$ implies that $\iota(\gamma)=e_{1} \neq \mu(d)$. Since $e_{1}$ is either $\mu^{-1}\left(u_{1}\right)$ or $u_{1}, \mu^{-1}\left(u_{1}\right) \neq d$. This implies that the embedding function $\varphi$ for $\gamma \preceq \gamma^{\prime}$ satisfies $\varphi(1)>1$. Thus the same function $\varphi$ regarded as a map $\{0,1, \ldots, \ell(\gamma)+$ $1\} \rightarrow\left\{0,2,3, \ldots, \ell\left(\gamma^{\prime}\right)+1\right\}$ is an embedding function for $b_{\ell}(\gamma)=\gamma \prec b_{\ell}\left(\gamma^{\prime}\right)$. And we obtain a descending closure operator $b_{\ell}: F_{\ell} \tau^{-1}(c) \rightarrow F_{\ell} \tau^{-1}(c)$ with image $F_{\ell-1} \tau^{-1}(c)$.

Again, by Corollary A.22, $B F_{\ell-1} \tau^{-1}(c)$ is a deformation retract of $B F_{\ell} \tau^{-1}(c)$.

Example 3.32. We continue with Example 3.27. Let us compute $\left[v_{0}\right] \downarrow \tau$ and $\left[v_{1}, v_{2}\right] \downarrow \tau$. As sets, we have

$$
\begin{aligned}
\left(\left[v_{0}\right] \downarrow \tau\right)_{0} & =\left\{\left(\gamma_{0}, \gamma_{0}\right),\left(\gamma_{0}, \gamma_{1}\right),\left(\gamma_{0}, \gamma_{2}\right),\left(\gamma_{0}, \gamma_{01}\right),\left(\gamma_{0}, \gamma_{02}\right),\left(\gamma_{1}, \gamma_{12}\right),\left(\gamma_{2}, \gamma_{12}\right)\right\} \\
\left(\left[v_{1}, v_{2}\right] \downarrow \tau\right)_{0} & =\left\{\left(\gamma_{12}, \gamma_{12}\right)\right\} .
\end{aligned}
$$

By $\gamma_{0} \prec \gamma_{01}, \gamma_{02}, \gamma_{1} \prec \gamma_{01}$ and $\gamma_{2} \prec \gamma_{02}$, the partial order on $\left[v_{0}\right] \downarrow \tau$ is given by

$$
\begin{array}{lll}
\left(\gamma_{0}, \gamma_{0}\right) & \prec\left[v_{0}\right] & \left(\gamma_{0}, \gamma_{01}\right) \\
\left(\gamma_{0}, \gamma_{0}\right) & \prec\left[v_{0}\right] & \left(\gamma_{0}, \gamma_{02}\right) \\
\left(\gamma_{0}, \gamma_{1}\right) & \prec\left[v_{0}\right] & \left(\gamma_{0}, \gamma_{01}\right) \\
\left(\gamma_{0}, \gamma_{2}\right) & \prec\left[v_{0}\right] & \left(\gamma_{0}, \gamma_{02}\right) .
\end{array}
$$

By $\gamma_{1} \prec \gamma_{12}$ and $\gamma_{2} \prec \gamma_{12}$, we have

$$
\begin{array}{lll}
\left(\gamma_{0}, \gamma_{1}\right) & \prec^{\left[v_{0}\right]} & \left(\gamma_{1}, \gamma_{12}\right) \\
\left(\gamma_{0}, \gamma_{2}\right) & \prec^{\left[v_{0}\right]} & \left(\gamma_{2}, \gamma_{12}\right) .
\end{array}
$$

The Hasse diagram of $\left[v_{0}\right] \downarrow \tau$ is given by

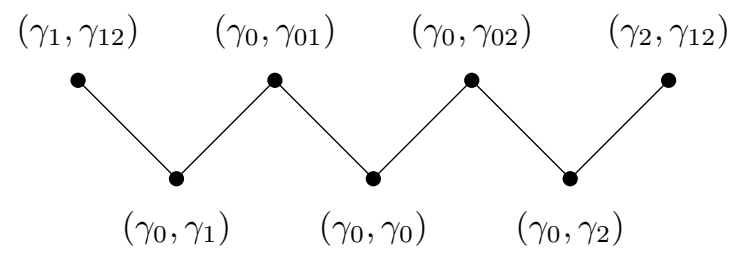

Thus both $B\left(\left[v_{0}\right] \downarrow \tau\right)$ and $B\left(\left[v_{0}, v_{1}\right] \downarrow \tau\right)$ are contractible. Note that $\tau^{-1}\left(\left[v_{0}\right]\right)$ is the subposet given by the zigzag between $\left(\gamma_{0}, \gamma_{1}\right)$ and $\left(\gamma_{0}, \gamma_{2}\right)$ and embedded in $\left[v_{0}\right] \downarrow \tau$ as a deformation retract.

\subsection{The Face Poset of Stable Subdivision}

We have constructed a subdivision $\operatorname{Sd}_{\mu}(X)$ of $X$ by using flow paths in $\S 2.3$. It turns out that the face poset of the stable subdivision $\operatorname{Sd}_{\mu}(X)$ is isomorphic to the poset of reduced flow paths $\overline{\mathrm{FP}}(\mu)$.

The aim of this section is to complete the proof of Theorem 1.2 by proving this fact. To this end, we need to understand relations between partial order on $\overline{\mathrm{FP}}(\mu)$ and the deformation retraction $R_{u}: \bar{u} \rightarrow d^{c}$ defined for each matched pair $d \prec_{1} u=\mu(d)$. 
Definition 3.33. For a reduced flow path $\gamma=\left(e_{1}, u_{1}, \ldots, e_{n}, u_{n} ; c\right)$, define a sequence of subpaths $\gamma^{(1)}, \ldots, \gamma^{(n+1)}$ of $\gamma$ by

$$
\gamma^{(i)}=\left(e_{i}, u_{i}, \ldots, e_{n}, u_{n} ; c\right)
$$

for $i \leq n$ and $\gamma^{(n+1)}=(c)$.

Let $i_{1}, \ldots, i_{k}$ be the indices of $e_{i}$ 's with $e_{i} \in D(\mu)$ and define

$$
W_{\gamma}^{s}=\bigcup_{i=1}^{n+1} e_{\gamma^{(i)}} \cup \bigcup_{\ell=1}^{k} e_{u\left(\gamma^{\left(i_{\ell}\right)}\right)}
$$

for $\ell=1, \ldots, k$, where $u\left(\gamma^{\left(i_{\ell}\right)}\right)$ is the operation on flow paths defined in Example 2.22. This is called the stable subspace along $\gamma$.

Example 3.34. Consider the partial matching on a 2-simplex in Example 2.23, in which we have shown $\gamma \prec \delta$ for the flow paths $\gamma=\left(e_{1}, e_{2}, e_{5}, e_{6} ; e_{7}\right)=\left(d_{1}, u_{1}, d_{2}, u_{2} ; c\right)$ and $\delta=$ $\left(e_{1}, e_{2}, e_{4}, e_{4}, e_{5}, e_{6} ; e_{7}\right)=\left(d_{1}^{\prime}, u_{1}^{\prime}, u_{2}^{\prime}, u_{2}^{\prime}, d_{3}^{\prime}, u_{3}^{\prime} ; c\right)$.

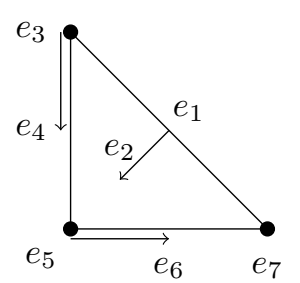

Figure 5: A partial matching on 2-simplex

The subpaths in Definition 3.33 for $\gamma$ are

$$
\begin{aligned}
& \gamma^{(1)}=\left(e_{1}, e_{2}, e_{5}, e_{6} ; c\right) \\
& \gamma^{(2)}=\left(e_{5}, e_{6} ; c\right) \\
& \gamma^{(3)}=(c) .
\end{aligned}
$$

Thus

$$
W_{\gamma}^{s}=e_{\gamma^{(1)}} \cup e_{\gamma^{(2)}} \cup e_{\gamma^{(3)}} \cup e_{u\left(\gamma^{(1)}\right)} \cup e_{u\left(\gamma^{(2)}\right)},
$$

which is the one dimensional complex drawn by dotted lines in Figure 6 .

Note that we need to add $e_{u\left(\gamma^{(1)}\right)}$ and $e_{u\left(\gamma^{(2)}\right)}$ to obtain a connected region.

Similarly

$$
\begin{aligned}
\delta^{(1)}=\delta & =\left(e_{1}, e_{2}, e_{4}, e_{4}, e_{5}, e_{6} ; c\right) \\
\delta^{(2)} & =\left(e_{4}, e_{4}, e_{5}, e_{6} ; c\right) \\
\delta^{(3)} & =\left(e_{5}, e_{6} ; c\right) \\
\delta^{(4)} & =(c) .
\end{aligned}
$$

Thus

$$
W_{\delta}^{s}=e_{\delta^{(1)}} \cup e_{\delta^{(2)}} \cup e_{\delta^{(3)}} \cup e_{\delta^{(4)}} \cup e_{u\left(\delta^{(1)}\right)} \cup e_{u\left(\delta^{(3)}\right)},
$$

which is the union of the shaded area and dotted lines in Figure 7.

From these figures, we see that $W_{\gamma}^{s} \subset \overline{W_{\delta}^{s}}$. 


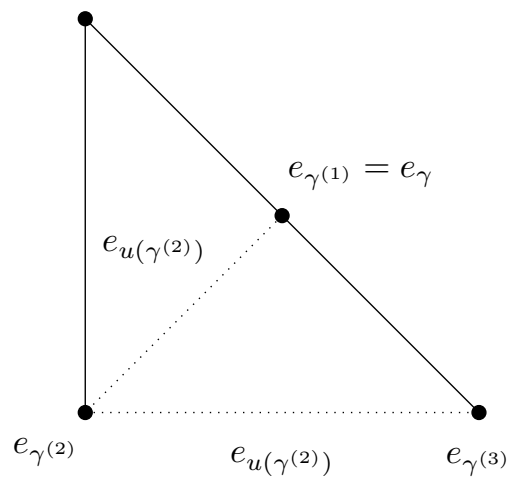

Figure 6: Stable subspace along $\gamma$

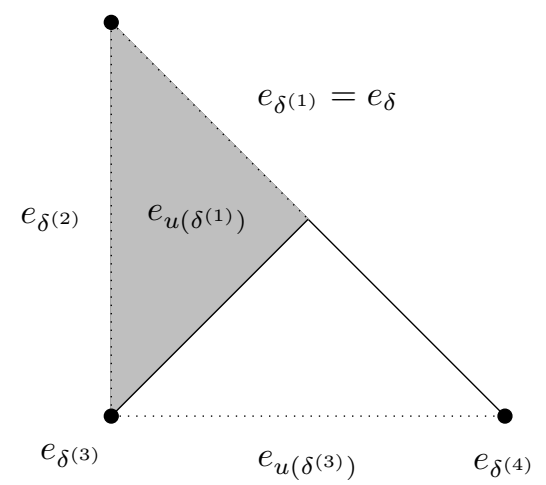

Figure 7: Stable subspace along $\delta$

Lemma 3.35. For $x \in e_{\gamma}$, the continuous flow $L_{x}$ stays in $W_{\gamma}^{s}$. Furthermore the flows $\left\{L_{x}\right\}_{x \in \iota(\gamma)}$ can be glued together to give rise to a continuous flow on $W_{\gamma}^{s}$.

$$
L_{\gamma}: W_{\gamma}^{s} \times[0, h] \longrightarrow W_{\gamma}^{s} .
$$

Proof. The first statement is obvious from the definition. For a reduced flow path $\gamma=\left(e_{1}, u_{1}, \ldots, e_{n}, u_{n} ; c\right)$, let $h=h_{x}$ for $x \in e_{u(\gamma)}$. This is independent of $x$, since the initial cell of $u(\gamma)$ belongs to $D(\mu)$.

Similarly define $h_{2}=h_{y}$ for $y \in e_{u\left(\gamma_{2}\right)}$ and define a map

$$
L_{1}: W_{\gamma}^{s} \times\left[h_{2}, h\right] \longrightarrow W_{\gamma}^{s}
$$

by

$$
L_{1}(x, t)=L_{d_{1}, u_{1}}\left(x, t-h_{2}\right),
$$

where $d_{1}=\mu^{-1}\left(u_{1}\right)$. This is a deformation retraction onto $W_{\gamma_{2}}^{s}$. By iterating this process, we obtain a sequence of homotopies $\left\{L_{i}: W_{\gamma_{i}}^{s} \times\left[h_{i+1}, h_{i}\right] \rightarrow W_{\gamma_{i}}^{s}\right\}$. By concatenating these homotopies we obtain a continuous map

$$
L: W_{\gamma}^{s} \times[0, h] \longrightarrow W_{\gamma}^{s}
$$

whose restriction to $\{x\} \times\left[0, h_{x}\right]$ coincides with $L_{x}$. 
By using $L_{\gamma}$, we have the following extension of the description of Lemma 2.43.

Lemma 3.36. For a reduced flow path $\gamma=\left(e_{1}, u_{1}, \ldots, e_{n}, u_{n} ; c\right)$, we have

$$
e_{\gamma^{(k)}}=\left\{x \in e_{k} \mid \exists \text { s.t. } L_{\gamma}(x, t) \in e_{\gamma^{(k+1)}}\right\}
$$

for $1 \leq k \leq n$.

Proof. By definition.

The stable subspace $W^{s}(c)$ introduced in Definition 2.48 for a critical cell $c$ decomposes into a union

$$
W^{s}(c)=\bigcup_{\tau(\gamma)=c} W_{\gamma}^{s} .
$$

The flows $L_{\gamma}$ for $\gamma$ with $\tau(\gamma)=c$ can be glued together to give rise to a flow on $W^{s}(c)$

$$
L_{c}: W^{s}(c) \times[0, h] \longrightarrow W^{s}(c) .
$$

The main result of this section is the following theorem, which establishes that the map $\overline{\mathrm{FP}}(\mu) \rightarrow F\left(\operatorname{Sd}_{\mu}(X)\right)$ given by $\gamma \mapsto e_{\gamma}$ is an isomorphism of posets.

Proposition 3.37. For $\gamma, \delta \in \overline{\mathrm{FP}}(\mu)$, we have $\gamma \preceq \delta$ if and only if $e_{\gamma} \subset \overline{e_{\delta}}$.

Assume the existence of a pair of reduced flow paths

$$
\begin{aligned}
& \gamma=\left(e_{1}, u_{1}, e_{2}, u_{2}, \ldots, e_{n}, u_{n} ; c\right), \text { and } \\
& \delta=\left(e_{1}^{\prime}, u_{1}^{\prime}, e_{2}^{\prime}, u_{2}^{\prime}, \ldots, e_{m}^{\prime}, u_{m}^{\prime} ; c^{\prime}\right)
\end{aligned}
$$

so that $\gamma \preceq \delta$ holds with the embedding function $\varphi:\{0,1, \ldots, k\} \rightarrow\{0,1, \ldots, m+1\}$. We also denote $d_{i}=\mu^{-1}\left(u_{i}\right)$ and $d_{j}^{\prime}=\mu^{-1}\left(u_{j}^{\prime}\right)$ for each $i$ and $j$, and recall for the reader's convenience the fact that a deformation retraction $R_{u}: \bar{u} \rightarrow \partial u \backslash d$ has been chosen for each matched cell pair $u=\mu(d)$ and used to define a continuous flow in the sense of Definition 2.40.

Lemma 3.38. The cell $e_{j}$ is fixed pointwise by the deformation retraction $R_{u_{\ell}^{\prime}}: \overline{u_{\ell}^{\prime}} \rightarrow \partial u_{\ell}^{\prime} \backslash d_{\ell}^{\prime}$ for each $1 \leq j \leq k$ and $\phi(j-1) \leq \ell<\phi(j)$.

Proof. Since $\phi$ is an embedding function, we have $e_{j} \preceq e_{p}^{\prime}$ for $\phi(j-1)<p \leq \phi(j)$. But since $\delta$ is a reduced flow path, any such $e_{p}^{\prime}$ is a face of the subsequent $u_{p+1}^{\prime}$ different from $d_{p+1}^{\prime}$, and all of its points must therefore be fixed by $R_{u_{p+1}^{\prime}}$. For these values of $p$, note that $\ell=p+1$ satisfies $\phi(j-1) \leq \ell<\phi(j)$.

Proposition 3.39. For every $1 \leq j \leq k$ and $\phi(j-1) \leq \ell<\phi(j)$, the following statements hold.

1. If $e_{\gamma^{(j)}} \subset \overline{e_{\delta^{(\ell+1)}}}$, then $e_{\gamma^{(j)}} \subset \overline{e_{\delta^{(\ell)}}}$ for every $\phi(j-1) \leq \ell<\phi(j)$.

2. If $e_{\gamma^{(j)}} \subset \overline{e_{\delta^{(\phi(j-1))}}}$, then $e_{\gamma^{(j-1)}} \subset \overline{e_{\delta^{(\phi(j-1)-1)}}}$.

Proof. To see that (1) holds, recall from Lemma 3.36 that $e_{\delta^{(\ell)}}$ consists of precisely those points of $e_{\ell}^{\prime}$ which are mapped to $e_{\delta^{(\ell+1)}}$ by the deformation retraction $R_{u_{\ell}^{\prime}}$. By assumption, $e_{j} \subset \overline{e_{\delta^{(\ell+1)}}}$ and by Lemma 3.38, the cell $e_{j}$ is fixed by these retractions for all $\ell$ in the stated range. Thus, we have

$$
e_{\gamma^{(j)}} \subset e_{j} \subset \overline{e_{\delta^{(\ell)}}} .
$$

Turning to (2), note that $u_{j-1}=u_{\phi(j-1)}^{\prime}$ since $\phi$ is an embedding function. Therefore, the retraction $R_{u_{j-1}}$ which defines $e_{\gamma^{(j-1)}}$ from $e_{\gamma^{(j)}}$ coincides exactly with the retraction $R_{u_{\phi(j-1)}^{\prime}}$ which similarly defines $e_{\delta(\phi(j-1)-1)}$. The desired inclusion $e_{\gamma^{(j-1)}} \subset \overline{e_{\delta(\phi(j-1)-1)}}$ now follows from $e_{\gamma^{(j)}} \subset \overline{e_{\delta^{(\phi(j-1))}}}$. 
Remark 3.40. It is worth noting that the argument in the above proof can be used to prove that $L_{\gamma}$ is a part of $L_{\delta}$ when $\gamma \preceq \delta$. Namely, if $x \in e_{\gamma}, L_{\delta}(x, t)=L_{\gamma}\left(x, t^{\prime}\right)$ for some $t^{\prime}$.

Recall that $e_{k} \prec c^{\prime}$ because $\phi$ is an embedding function. Proposition 3.39, along with the initial condition

$$
e_{\gamma^{(k)}} \subset e_{k} \subset \overline{c^{\prime}}=\overline{e_{\delta^{(\phi(k))}}}
$$

proves one half of Proposition 3.37 by reverse-induction on $j \in\{1, \ldots, k\}$ (recall that $\gamma=\gamma^{(0)}$ and similarly for $\delta$ ). Conversely, suppose that $e_{\gamma} \subset \overline{e_{\delta}}$. In order to prove $\gamma \preceq \delta$, we need to find an embedding function $\varphi$.

Define a sequence of nonnegative integers $i_{0}=0, i_{1}, i_{2}, \ldots$ inductively by

$$
i_{j}= \begin{cases}\max \left\{p>i_{j-1} \mid e_{\gamma^{(j)}} \subset \overline{e_{\delta^{(p)}}}\right\}, & \text { if }\left\{p>i_{j-1} \mid e_{\gamma^{(j)}} \subset \overline{e_{\delta^{(p)}}}\right\} \neq \emptyset, \\ m+1, & \text { otherwise }\end{cases}
$$

and set $k=\min \left\{j \mid i_{j}=m+1\right\}$. Then we obtain a strictly increasing function

$$
\varphi:\{0, \ldots, k\} \longrightarrow\{0, \ldots, m+1\}
$$

by $\varphi(j)=i_{j}$.

Let us verify that this is an embedding function for $\gamma \preceq \delta$. By definition, it is a strictly increasing function with $\varphi(0)=0$ and $\varphi(k)=m+1$. It remains to prove that

1. $u_{j}=u_{\varphi(j)}^{\prime}$ for each $1 \leq j<k$, and

2. for each $1 \leq j \leq k, e_{j} \preceq e_{p}^{\prime}$ for all $\varphi(j-1)<p \leq \varphi(j)$.

The second part is immediate. By definition, $e_{\gamma^{(j)}} \subset \overline{e_{\delta^{(p)}}}$ for $\varphi(j-1)<p \leq \varphi(j)$. But $e_{\gamma^{(j)}} \subset e_{j}$ and $e_{\delta^{(p)}} \subset \overline{e_{p}^{\prime}}$, which imply that $e_{j} \cap \overline{e_{p}^{\prime}} \neq \emptyset$. Thus we have $e_{j} \preceq e_{p}^{\prime}$.

For the first part, let us first prove that, for each $j, e_{\gamma^{(j)}} \not \subset\left(d_{\varphi(j)}^{\prime}\right)^{c}$. Suppose $e_{\gamma^{(j)}} \subset\left(d_{\varphi(j)}^{\prime}\right)^{c}$. Then $R_{u_{\varphi(j)}^{\prime}}\left(e_{\gamma^{(j)}}\right)=e_{\gamma^{(j)}}$ by the definition of the retraction $R_{u_{\varphi(j)}^{\prime}}: \overline{u_{\varphi(j)}^{\prime}} \rightarrow\left(d_{\varphi(j)}^{\prime}\right)^{c}$. Since $e_{\gamma^{(j)}} \subset \frac{\varphi(j)}{e_{\delta(\varphi(j))}}$, we have

$$
e_{\gamma^{(j)}}=R_{u_{\varphi(j)}^{\prime}}\left(e_{\gamma^{(j)}}\right) \subset R_{u_{\varphi(j)}^{\prime}}\left(\overline{e_{\delta(\varphi(j))}}\right)=\overline{e_{\delta(\varphi(j)+1)}},
$$

which contracts to our choice of $\varphi(j)$. Hence $e_{\gamma^{(j)}} \not \subset\left(d_{\varphi(j)}^{\prime}\right)^{c}$ or

$$
e_{\gamma^{(j)}} \subset \overline{u_{\varphi(j)}^{\prime}} \backslash\left(d_{\varphi(j)}^{\prime}\right)^{c}=d_{\varphi(j)}^{\prime} \cup u_{\varphi(j)}^{\prime} .
$$

If $e_{\gamma^{(j)}} \subset u_{\varphi(j)}^{\prime}, u_{\varphi(j)}^{\prime} \cap e_{j} \neq \emptyset$ and we obtain $e_{j}=u_{j}=u_{\varphi(j)}^{\prime}$. When $e_{\gamma^{(j)}} \subset d_{\varphi(j)}^{\prime}, d_{\varphi(j)}^{\prime} \cap e_{j} \neq \emptyset$ and we have $e_{j}=d_{j}=d_{\varphi(j)}^{\prime}$. Apply the matching $\mu$, and we obtain $u_{j}=u_{\varphi(j)}^{\prime}$.

This concludes the proof of Proposition 3.37, and we have the following desired consequence.

Corollary 3.41. The map $\overline{\mathrm{FP}}(\mu) \rightarrow F\left(\mathrm{Sd}_{\mu}(X)\right)$ given by $\gamma \mapsto e_{\gamma}$ is an isomorphism of posets.

Proof of Theorem 1.2. The isomorphism in Corollary 3.41 induces a homeomorphism between classifying spaces $B \overline{\mathrm{FP}}\left(\mu_{f}\right) \cong B F\left(\mathrm{Sd}_{\mu_{f}}(X)\right)$. It is well known that the composition $B F$ is nothing but the barycentric subdivision ${ }^{6} \mathrm{Sd}$ and we obtain a homeomorphism

$$
B \overline{\mathrm{FP}}\left(\mu_{f}\right) \cong \operatorname{Sd}\left(\operatorname{Sd}_{\mu_{f}}(X)\right) \cong X
$$

By combining the homotopy equivalences in Theorem 3.31, Theorem A.35, and Theorem 3.25 with this homeomorphism, we obtain a homotopy equivalence

$$
X \cong B \overline{\mathrm{FP}}\left(\mu_{f}\right) \simeq B^{n c l} \bar{C}\left(\mu_{f}\right) \simeq B^{2} \bar{C}\left(\mu_{f}\right) \simeq B^{2} C\left(\mu_{f}\right)
$$

\footnotetext{
${ }^{6}$ See, for example, section 12.4 of Björner's article [Bjö95] or section 10.3.5 of Kozlov's book [Koz08].
} 


\section{A Homotopy Theory of Small Categories}

Our main technical tool in this paper is homotopy theory of small categories, including 2categories. We collect definitions and important properties in homotopy theory of small categories used in this paper for the convenience of the reader.

Our main references include $\$ 11$ of May's book [May72], Dugger's exposition [Dug08] on homotopy colimits, and Segal's papers [Seg68, Seg73, Seg74].

\section{A.1 Simplicial Sets and Simplicial Spaces}

In homotopy theory of small categories, each small category $C$ is made into a topological space by the classifying space construction, which is defined as the geometric realization of a simplicial set $N C$, called the nerve of $C$. When $C$ is a topological category, the nerve $N C$ is a simplicial space.

In this section, we recall homotopy theoretic properties of simplicial spaces, including simplicial sets.

Definition A.1. A simplicial object in a category $\boldsymbol{C}$ consists of a sequence of objects $\left\{X_{n}\right\}_{n=0,1, \ldots}$ in $\boldsymbol{C}$ and morphisms

$$
\begin{aligned}
& d_{i}: X_{n} \longrightarrow X_{n-1} \\
& s_{i}: X_{n} \longrightarrow X_{n+1}
\end{aligned}
$$

for $0 \leq i \leq n$ satisfying the following relations:

1. $d^{j} \circ d^{i}=d^{i} \circ d^{j-1}$ for $i<j$

2. $s^{j} \circ d^{i}=d^{i} \circ s^{j-1}$ for $i<j$

3. $s^{j} \circ d^{j}=1=s^{j} \circ d^{j+1}$

4. $s^{j} \circ d^{i}=d^{i-1} \circ s^{j}$ for $i>j+1$

5. $s^{j} \circ s^{i}=s^{i} \circ s^{j+1}$ for $i \leq j$.

Simplicial objects in the categories of sets and topological spaces are called simplicial sets and simplicial spaces, respectively.

It is convenient to introduce the following small category.

Definition A.2. The category of isomorphism classes of finite totally ordered sets and orderpreserving maps is denoted by $\Delta$. The object of cardinality $n+1$ is denoted by $[n]$ and is identified with the subposet $0<1<\cdots<n$ of $\mathbb{Z}$.

The subcategory of injective morphisms is denoted by $\Delta_{\text {inj }}$.

Lemma A.3. The category of simplicial sets is isomorphic to the category $\mathbf{C a t}\left(\Delta^{\mathrm{op}}\right.$, Set $)$ of contravariant functors from $\Delta$ to Set. Similarly the category of simplicial spaces is isomorphic to the category $\mathbf{C a t}\left(\Delta^{\mathrm{op}}\right.$, Top) of contravariant functors from $\Delta$ to Top.

Definition A.4. We denote the categories of simplicial sets and simplicial spaces by $\mathbf{S e t}^{\Delta^{\mathrm{op}}}$ and $\mathbf{T o p}^{\Delta^{\mathrm{op}}}$, respectively. We regard $\mathbf{S e t}^{\Delta^{\mathrm{op}}}$ as a full subcategory of $\mathbf{T o p}^{\Delta^{\mathrm{op}}}$ consisting of simplicial spaces with discrete topology.

For a simplicial space $X$ and a morphism $\varphi:[m] \rightarrow[n]$ in $\Delta$, the induced map is denoted by $\varphi^{*}: X_{n} \rightarrow X_{m}$. 
The following variation is useful when we study acyclic categories.

Definition A.5. A functor $X: \Delta_{\mathrm{inj}}^{\mathrm{op}} \rightarrow$ Top is called a $\Delta$-set.

Remark A.6. A $\Delta$-set $X$ can be regarded as a "simplicial set without degeneracies", i.e. it consists of a sequence of sets $\left\{X_{n}\right\}$ together with maps $d_{i}: X_{n} \rightarrow X_{n-1}$ satisfying the same relations as in the definition of simplicial sets. See [RS71] by Rourke and Sanderson, for more details on $\Delta$-sets.

There are two popular ways to form a topological space from a simplicial space.

Definition A.7. For a simplicial space $X$, define

$$
\begin{aligned}
& |X|=\left(\coprod_{n=0}^{\infty} X_{n} \times \Delta^{n}\right) / \sim \\
& \|X\|=\left(\coprod_{n=0}^{\infty} X_{n} \times \Delta^{n}\right) / \sim_{\text {inj }},
\end{aligned}
$$

where $\Delta^{n}$ is the standard $n$-simplex whose vertices are identified with elements in $[n]$ and $\sim$ is the equivalence relation generated by $\left(\varphi^{*}(x), s\right) \sim\left(x, \varphi_{*}(s)\right)$ for $x \in X_{n}, s \in \Delta^{m}$, and $\varphi \in \Delta([m],[n])$. The map $\varphi_{*}: \Delta^{m} \rightarrow \Delta^{n}$ is the affine map induced by the map between vertices $\varphi:[m] \rightarrow[n]$. The equivalence relation $\sim_{\text {inj }}$ is generated by $\left(\varphi^{*}(x), s\right) \sim\left(x, \varphi_{*}(s)\right)$ for $\varphi \in \Delta_{\mathrm{inj}}([m],[n])$.

$|X|$ and $\|X\|$ are called the (geometric) realization and the fat realization of $X$, respectively. The canonical quotient map from the fat realization to the realization is denoted by

$$
q_{X}:\|X\| \longrightarrow|X| .
$$

One of the advantages of the fat realization $\|X\|$ is the following homotopy invariance.

Proposition A.8. For a morphism $f: X \rightarrow Y$ of simplicial spaces whose $n$-th stage $f_{n}: X_{n} \rightarrow$ $Y_{n}$ is a homotopy equivalence for all $n$, the induced map on fat realizations

$$
\|f\|:\|X\| \longrightarrow\|Y\|
$$

is a homotopy equivalence.

Proof. See Appendix A of Segal's paper [Seg74], for example.

Thus the realization is homotopy invariant for simplicial spaces $X$ whose realization $|X|$ is homotopy equivalent to the fat realization $\|X\|$. The following is a sufficient condition for the quotient $q_{X}:\|X\| \rightarrow|X|$ to be a homotopy equivalence.

Definition A.9. For a simplicial space $X$, define the $n$-th latching space $L_{n} X$ by

$$
L_{n} X=\bigcup_{i=0}^{n-1} s_{i}\left(X_{n-1}\right) .
$$

A simplicial space $X$ is said to be cofibrant if the inclusion $i_{n}: L_{n} X \hookrightarrow X_{n}$, the $n$-th latching map, is a closed cofibration for all $n$. 
Remark A.10. For a pair $(X, A)$ of topological spaces, the inclusion $A \hookrightarrow X$ is a closed cofibration if and only if $(X, A)$ is an NDR pair, i.e. there exist a continuous function $u: A \rightarrow[0,1]$ and a homotopy $h: X \times[0,1] \rightarrow X$ such that $A=u^{-1}(0), h(x, t)=(x, t)$ for $(x, t) \in X \times\{0\} \cup A \times[0,1]$, and $h(x, 1) \in A$ for $x \in h^{-1}([0,1))$.

Recall that one of the most important examples of closed cofibrations is an inclusion of a subcomplex in a CW complex.

Example A.11. If $X$ is a simplicial space consisting of CW complexes in which the latching space $L_{n} X$ is a subcomplex of $X_{n}$ for all $n$, then $X$ is cofibrant.

In particular, for a 2-category $C$ the nerve $N B C$ of the associated topological category $B C$ is a cofibrant simplicial space.

Proposition A.12. When $X$ is a cofibrant simplicial space, the quotient map $q_{X}:\|X\| \rightarrow|X|$ is a homotopy equivalence.

Proof. See also Appendix A of Segal's paper [Seg74].

Corollary A.13. Let $f: X \rightarrow Y$ be a map of simplicial spaces. Suppose $X$ and $Y$ are cofibrant and that $f_{n}: X_{n} \rightarrow Y_{n}$ is a homotopy equivalence for all $n$. Then the induced map $|f|:|X| \rightarrow|Y|$ is a homotopy equivalence.

\section{A.2 Nerves and Classifying Spaces}

The nerve construction transforms small categories to simplicial sets, and then to topological spaces by taking the geometric realization functor. The nerve construction can be extended to 2-categories and topological categories by using simplicial spaces.

Definition A.14. For a small category $C$, define

$$
N_{n}(C)=\left\{\left(u_{n}, \ldots, u_{1}\right) \in C_{1}^{n} \mid s\left(u_{n}\right)=t\left(u_{n-1}\right), \ldots, s\left(u_{2}\right)=t\left(u_{1}\right)\right\} .
$$

Elements of $N_{n}(C)$ are called $n$-chains.

Lemma A.15. For a small category $C$, these sets $\left\{N_{n}(C)\right\}_{n \geq 0}$ together with maps

$$
\begin{array}{ll}
d_{i}: & N_{n}(C) \longrightarrow N_{n-1}(C) \\
s_{i}: & N_{n}(C) \longrightarrow N_{n+1}(C)
\end{array}
$$

defined by

$$
\begin{aligned}
& d_{i}\left(u_{n}, \cdots, u_{1}\right)= \begin{cases}\left(u_{n}, \ldots, u_{2}\right) & i=0 \\
\left(u_{n}, \ldots, u_{i+2}, u_{i+1} \circ u_{i}, \ldots, u_{1}\right), & 1 \leq i \leq n-1 \\
\left(u_{n-1}, \ldots, u_{1}\right), & i=n,\end{cases} \\
& s_{i}\left(u_{n}, \cdots, u_{1}\right)= \begin{cases}\left(u_{n}, \ldots, u_{1}, 1_{s\left(u_{1}\right)}\right), & i=0 \\
\left(u_{n}, \ldots, u_{i+1}, 1_{t\left(u_{i}\right)}, u_{i}, \ldots, u_{1}\right) & 1 \leq i \leq n\end{cases}
\end{aligned}
$$

form a simplicial set.

Definition A.16. The simplicial set $N(C)$ is called the nerve of $C$. The geometric realization of the nerve $N(C)$ is denoted by $B C$ and is called the classifying space of $C$. 
Remark A.17. When $C$ is a poset, $B C$ agrees with the geometric realization of the order complex of $C$.

The following fact is fundamental.

Lemma A.18. Regard the totally ordered set $[n]=\{0<1<\cdots<n\}$ as a small category. Then, for a small category $C$, we have a natural isomorphism of sets

$$
N_{n}(C) \cong \mathbf{C a t}([n], C) .
$$

Furthermore, under the identification between simplicial sets and functors $\Delta^{\mathrm{op}} \rightarrow$ Set, the nerve construction defines a functor

$$
N: \mathbf{C a t} \longrightarrow \text { Set }^{\Delta^{\mathrm{op}}},
$$

where $\mathbf{S e t}^{\Delta^{\mathrm{op}}}$ is the category of simplicial sets.

Thus the classifying space construction is also a functor

$$
B: \text { Cat } \stackrel{N}{\longrightarrow} \text { Set }^{\Delta^{o p}} \stackrel{|-|}{\longrightarrow} \text { Top . }
$$

where $|-|$ denotes the geometric realization functor. In particular, any functor $f: C \rightarrow D$ induces a continuous map of topological spaces $B f: B C \rightarrow B D$.

A natural transformation between functors induces a homotopy.

Lemma A.19. A natural transformation $\theta: f \Rightarrow g$ between functors $f, g: C \rightarrow D$ induces a homotopy

$$
B \theta: B C \times[0,1] \longrightarrow B D
$$

between $B f$ and $B g$.

As a corollary, we obtain the following well-known but useful fact.

Corollary A.20. Suppose a functor $f: C \rightarrow D$ between small categories has a right or a left adjoint $g: D \rightarrow C$. Then $B f: B C \rightarrow B D$ is a homotopy equivalence. In particular, when $C$ has an initial or a terminal object, $B C$ is contractible.

Proof. Suppose $g$ is right adjoint to $f$. We have natural transformations

$$
\begin{aligned}
1_{C} & \Longrightarrow g \circ f \\
f \circ g & \Longrightarrow 1_{D} .
\end{aligned}
$$

By Lemma A.19, they induce homotopies

$$
\begin{aligned}
& B g \circ B f=B(g \circ f) \simeq B 1_{C}=1_{B C} \\
& B f \circ B g=B(f \circ g) \simeq B 1_{D}=1_{B D}
\end{aligned}
$$

and thus $B g$ is a homotopy inverse of $B f: B C \rightarrow B D$.

When $C$ has an initial object $*$, the collapsing functor $p: C \rightarrow *$ is a right adjoint to the inclusion $* \hookrightarrow C$. And we have $B C \simeq B *=*$.

Furthermore we obtain a deformation retraction in the following special case.

Definition A.21. A map of poset $f: P \rightarrow P$ is called a descending closure operator if $f \circ f=f$ and $f(x) \leq x$ for all $x \in P$. Dually it is called an ascending closure operator if $f \circ f=f$ and $f(x) \geq x$ for all $x \in P$. 
Corollary A.22. If a poset $P$ has a descending or an ascending closure operator $f: P \rightarrow P$, $B(f(P))$ is a strong deformation retract of $B P$.

Proof. Regard $P$ as a small category. A poset map $f: P \rightarrow P$ is an endofunctor. Let $i: f(P) \hookrightarrow$ $P$ be the inclusion. When $f$ is a descending closure operator, we have $f \circ i=1_{f(P)}$. Furthermore the relation $f(x) \leq x$ gives rise to a natural transformation $i \circ f \Rightarrow 1_{P}$, which is identity on $f(P)$. Thus we have a homotopy $B i \circ B f \simeq 1_{B P}$ which is identity on $B f(P)$.

When $f$ is a descending closure operator, we have a natural transformation $1_{P} \Rightarrow i \circ f$ and the same conclusion holds.

Remark A.23. It can be proved that $B P$ collapses onto $B(f(P))$ under the same assumption. See $\$ 13.2$ of Kozlov's book [Koz08].

When $C$ is acyclic, we only need nondegenerate chains.

Definition A.24. For a small category $C$, define

$$
\bar{N}_{n}(C)=N_{n}(C)-\bigcup_{i=0}^{n-1} s_{i}\left(N_{n-1}(C)\right) .
$$

Elements of $\bar{N}_{n}(C)$ are called nondegenerate $n$-chains.

Lemma A.25. When $C$ is acyclic, the face operators $d_{i}$ can be restricted to nondegenerate chains. Thus the collection $\bar{N}(C)=\left\{\bar{N}_{n}(C)\right\}_{n \geq 0}$ forms a $\Delta$-set.

Definition A.26. For an acyclic category $C$, elements of $\bar{N}_{n}(C)$ are called nondegenerate $n$ chains and the $\Delta$-set $\bar{N}(C)$ is called the nondegenerate nerve of $C$.

Lemma A.27. For a small acyclic category $C$, we have a natural homeomorphism

$$
B C=|N(C)| \cong\|\bar{N}(C)\|,
$$

where $\|-\|$ denotes the geometric realization of $\Delta$-sets.

It is straightforward to extend the construction of the classifying space to topological categories.

Lemma A.28. For a topological category $C, N C=\left\{N_{n}(C)\right\}_{n \geq 0}$ forms a simplicial space.

Definition A.29. For a topological category $C$, the geometric realization of the simplicial space $N C$ is called the classifying space of $C$ and is denoted by $B C$.

There are several ways to define the classifying space of a 2-category. A good reference is the paper [CCG10] by Carrasco, Cegarra, and Garzón in which they compare ten different nerves for bicategories (weak 2-categories).

One of the most classical constructions is the following bisimplicial nerve.

Definition A.30. For a 2-category $C$, define a topological category $B C$ by $(B C)_{0}=C_{0}$ and

$$
(B C)(x, y)=B(C(x, y))
$$

for $x, y \in C_{0}$. The classifying space of this topological category is denoted by $B^{2} C$ and is called the classifying space of $C$. 
$B^{2}$ obviously defines a functor

$$
B^{2}: \mathbf{C a t}_{2} \longrightarrow \text { Top }
$$

from the category $\mathbf{C a t}_{2}$ of 2-categories and 2-functors to the one of topological spaces and continuous maps.

Proposition A.31. If a strict 2-functor $f: C \rightarrow D$ induces a homotopy equivalence

$$
B f(x, y): B C(x, y) \longrightarrow B D(f(x), f(y))
$$

for all pairs of objects $x, y \in C_{0}$, then

$$
B^{2} f: B^{2} C \longrightarrow B^{2} D
$$

is a homotopy equivalence.

Proof. By assumption, $f$ induces a homotopy equivalence

$$
N_{n} B f: N_{n} B C \longrightarrow N_{n} B D
$$

for all $n$. Now the result follows from Lemma A.13 and Example A.11.

Note that the functor $B^{2}: \mathbf{C a t}_{2} \rightarrow$ Top, cannot be extended to lax or colax functors. In order to construct a classifying space which is functorial with respect to lax or colax functors, one of the ways is to modify the description in Lemma A.18.

Definition A.32. For a 2-category $C$, define

$$
\begin{aligned}
N_{n}^{c l}(C) & =\{\boldsymbol{u}:[n] \rightarrow C \mid \text { colax functors }\} \\
N_{n}^{n c l}(C) & =\{\boldsymbol{u}:[n] \rightarrow C \mid \text { normal colax functors }\} \\
N_{n}^{l}(C) & =\{\boldsymbol{u}:[n] \rightarrow C \mid \text { lax functors }\} \\
N_{n}^{n l}(C) & =\{\boldsymbol{u}:[n] \rightarrow C \mid \text { normal lax functors }\} .
\end{aligned}
$$

Lemma A.33. The collections $N^{c l}(C)=\left\{N_{n}^{c l}(C)\right\}_{n \geq 0}, N^{n c l} C=\left\{N_{n}^{n c l}(C)\right\}_{n \geq 0}, N^{l}(C)=$ $\left\{N_{n}^{l}(C)\right\}_{n \geq 0}$, and $N^{n l}(C)=\left\{N_{n}^{n l}(C)\right\}_{n \geq 0}$ form simplicial sets and we obtain functors

$$
\begin{aligned}
B^{c l} & : \mathbf{C a t}_{2, c l} \longrightarrow \mathbf{S e t}^{\Delta^{\mathrm{op}}} \\
B^{n c l} & : \mathbf{C a t}_{2, n c l} \longrightarrow \mathbf{S e t}^{\Delta^{\mathrm{op}}} \\
B^{l} & : \mathbf{C a t}_{2, l} \longrightarrow \mathbf{S e t}^{\Delta^{\mathrm{op}}} \\
B^{n l} & : \mathbf{C a t}_{2, n l} \longrightarrow \mathbf{S e t}^{\Delta^{\mathrm{op}}} .
\end{aligned}
$$

Remark A.34. The above construction is originally due to Duskin [Dus02] in the case of lax functors. See also a paper [Str96] by Street.

It is known that all of these constructions give rise to the same homotopy type.

Theorem A.35. We have weak homotopy equivalences

$$
B^{c l} C \simeq B^{n c l} C \simeq B^{2} C \simeq B^{n l} C \simeq B^{l} C .
$$

Proof. See the paper [CCG10] by Carrasco, Cegarra, and Garzón. 


\section{A.3 Comma Categories and Quillen's Theorem A}

Given a functor $f: C \rightarrow D$ between small categories, a basic question is when the induced map

$$
B f: B C \longrightarrow B D
$$

is a homotopy equivalence. In order to measure the difference between $C$ and $D$ via $f$, one might want to look at fibers of $f$.

Definition A.36. For a functor $f: C \rightarrow D$, the fiber $f^{-1}(y)$ over $y \in D_{0}$ is defined to be the subcategory of $C$ whose sets of objects and morphisms are given by

$$
\begin{aligned}
& f^{-1}(y)_{0}=f_{0}^{-1}(y)=\left\{x \in C_{0} \mid f_{0}(x)=y\right\} \\
& f^{-1}(y)_{1}=f_{1}^{-1}\left(1_{y}\right)=\left\{u \in C_{1} \mid f_{1}(u)=1_{y}\right\} .
\end{aligned}
$$

Unfortunately $B f$ fails to be a fibration in general, and genuine fibers do not tell us differences. The standard technique in homotopy theory suggests to take homotopy fibers. Quillen [Qui73] found that homotopy fibers of $B f$ can be described in terms of comma categories.

Definition A.37. For a small category $C$ and an object $x \in C_{0}$, define a category $C \downarrow x$ as follows. Objects are morphisms in $C$ of the form $u: y \rightarrow x$ :

$$
(C \downarrow x)_{0}=\left\{u \in C_{1} \mid t(u)=x\right\}=t^{-1}(x) .
$$

A morphism from $u: y \rightarrow x$ to $v: z \rightarrow x$ is a morphism $w: y \rightarrow z$ in $C$ satisfying $u=v \circ w$

$$
(C \downarrow x)(u, v)=\{w \in C(y, z) \mid u=v \circ w\} .
$$

Compositions of morphisms are given by compositions in $C$. Dually we define a category $x \downarrow C$ whose objects are morphisms in $C$ of the form $u: x \rightarrow y$. A morphism from $u: x \rightarrow y$ to $v: x \rightarrow z$ is a morphism $w: y \rightarrow z$ satisfying $v=w \circ u$.

More generally, given a functor $f: C \rightarrow D$ and an object $y \in D_{0}$, define a category $f \downarrow y$ as follows. The set of objects is given by

$$
(f \downarrow y)_{0}=\left\{(x, u) \in C_{0} \times D_{1} \mid u \in D(f(x), y)\right\} .
$$

The set of morphisms from $(x, u)$ to $\left(x^{\prime}, u^{\prime}\right)$ is given by

$$
(f \downarrow y)\left((x, u),\left(x^{\prime}, u^{\prime}\right)\right)=\left\{w \in C\left(x, x^{\prime}\right) \mid u=u^{\prime} \circ f(w)\right\} .
$$

Dually define a category $y \downarrow f$ by

$$
\begin{aligned}
(y \downarrow f)_{0} & =\left\{(u, x) \in D_{1} \times C_{0} \mid u \in C(y, f(x))\right\} \\
(y \downarrow f)\left((u, x),\left(u^{\prime}, x^{\prime}\right)\right) & =\left\{w \in C\left(x, x^{\prime}\right) \mid u^{\prime}=f(w) \circ u\right\} .
\end{aligned}
$$

The categories $f \downarrow x$ and $x \downarrow f$ are called left and right homotopy fibers of $f$ at $x$, respectively.

Remark A.38. The categories $f \downarrow x$ and $x \downarrow f$ are often called comma categories. The terminology used above is based on a homotopy-theoretic point of view. The name comma category actually refers to a more general construction. Categories defined in Definition A.37 are sometimes called coslice/slice categories and under/over categories.

Note that different authors use different notations for these categories. The notation $f \downarrow x$ and $x \downarrow f$ can be found in Mac Lane's book [ML98]. Quillen [Qui73] denotes them by $C / x, x \backslash C$, $f / y$, and $y \backslash f$, respectively. 
The following result is due to Quillen [Qui73] and is called Quillen's Theorem A.

Theorem A.39 (Theorem A). For a functor $f: C \rightarrow D$ between small categories, if $B(f \downarrow y)$ is contractible for all $y \in D_{0}$, then $B f: B C \rightarrow B D$ is a homotopy equivalence.

The same is true when $B(y \downarrow f)$ is contractible for all $y \in D_{0}$.

This fact says that the family of spaces $B(f \downarrow y)$ measures the difference of $B C$ and $B D$ via $B f$ in the homotopy category of topological spaces.

We close this section by describing relations between genuine fibers and homotopy fibers.

Definition A.40. For a functor $f: C \rightarrow D$ between small categories and an object $y \in D_{0}$, define functors

$$
\begin{aligned}
& i_{y}: \quad f^{-1}(y) \longrightarrow y \downarrow f \\
& j_{y}: \quad f^{-1}(y) \longrightarrow f \downarrow y
\end{aligned}
$$

by

$$
\begin{aligned}
& i_{y}(x)=\left(1_{y}, x\right) \\
& j_{y}(x)=\left(x, 1_{y}\right)
\end{aligned}
$$

on objects.

When $i_{y}$ has a right adjoint $s_{y}$ for each object $y$ in $D$, the functor $f$ is called prefibered or a prefibered category. The collection $\left\{s_{y}\right\}_{y \in D_{0}}$ is called a prefibered structure on $f$. Dually when $j_{y}$ has a left adjoint $t_{x}$ for each object $y$ in $D$, the functor $f$ is called precofibered or a precofibered category. The collection $\left\{t_{y}\right\}_{y \in D_{0}}$ is called a precofibered structure on $f$.

Corollary A.41. Suppose $f: C \rightarrow D$ is either prefibered or precofibered. If $B f^{-1}(y)$ is contractible for each $y \in D_{0}$, then $B f: B C \rightarrow B D$ is a homotopy equivalence.

\section{A.4 Comma Categories and Quillen's Theorem A for 2-Categories}

We need to extend Theorem A.39 to poset-categories and, more generally, to 2-categories for our purposes. We first need to define homotopy fibers or comma categories for colax functors. According to Cegarra [Ceg11], comma categories for 2-functors were introduced by Gray [Gra80], whose extension to colax functors can be found in del Hoyo's paper [dH12] $]^{7}$.

Definition A.42. Let $C$ and $D$ be 2-categories.

1. For a colax functor $f: C \rightarrow D$ and an object $y \in D_{0}$, define a 2-category $f \downarrow y$ as follows.

(a) The set of objects is given by

$$
(f \downarrow y)_{0}=\left\{(x, u) \in C_{0} \times D_{1} \mid u \in D(f(x), y)_{0}\right\} .
$$

(b) For $(x, u),\left(x^{\prime}, u^{\prime}\right) \in(f \downarrow y)_{0}$, the sets of objects and morphisms in the category $(f \downarrow y)\left((x, u),\left(x^{\prime}, u^{\prime}\right)\right)$ are defined by

$$
\begin{aligned}
(f \downarrow y)\left((x, u),\left(x^{\prime}, u^{\prime}\right)\right)_{0} & =\left\{(\theta, w) \in D_{2} \times C_{1} \mid \theta: u^{\prime} \circ f(w) \Rightarrow u\right\} \\
(f \downarrow y)\left((x, u),\left(x^{\prime}, u^{\prime}\right)\right)\left((\theta, w),\left(\theta^{\prime}, w^{\prime}\right)\right) & =\left\{\varphi \in C\left(x, x^{\prime}\right)\left(w^{\prime}, w\right) \mid \theta^{\prime}=\theta *\left(u^{\prime} \circ f(\varphi)\right)\right\} .
\end{aligned}
$$

\footnotetext{
${ }^{7}$ Note that colax functors in the sense of this paper are called "lax functors" in del Hoyo's paper.
} 


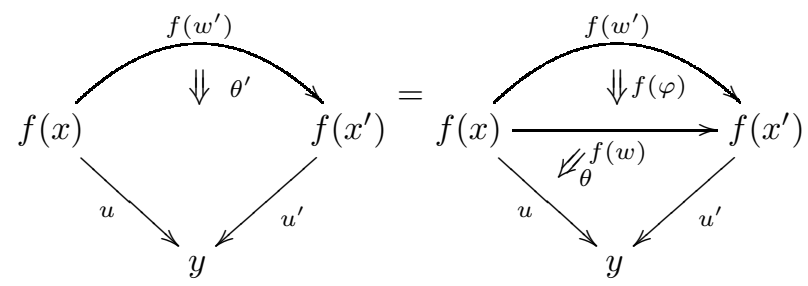

(c) For an object $(x, u) \in(f \downarrow y)_{0}$, define $1_{(x, u)}=\left(u^{\prime} \circ f_{x}, 1_{x}\right)$.

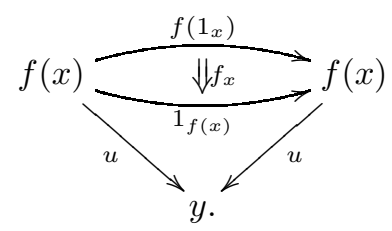

(d) The horizontal composition in $f \downarrow y$

$$
\circ:(f \downarrow y)\left(\left(x^{\prime}, u^{\prime}\right),\left(x^{\prime \prime}, u^{\prime \prime}\right)\right) \times(f \downarrow y)\left((x, u),\left(x^{\prime}, u^{\prime}\right)\right) \longrightarrow(f \downarrow y)\left((x, u),\left(x^{\prime \prime}, u^{\prime \prime}\right)\right)
$$

is defined by the following diagram

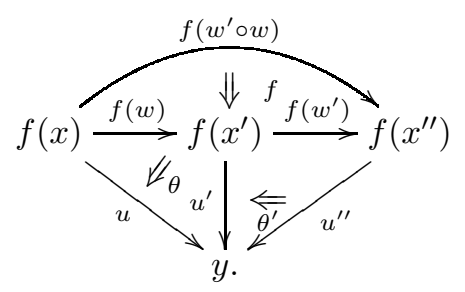

(e) The vertical composition in $f \downarrow y$ is given by that of $C$.

2. For a lax functor $f$ and an object $y \in D_{0}$, define a 2-category $y \downarrow f$ as follows.

(a) The set of objects is given by

$$
(y \downarrow f)_{0}=\left\{(u, x) \in D_{1} \times C_{0} \mid u \in D(y, f(x))_{0}\right\} .
$$

(b) For $(u, x),\left(u^{\prime}, x^{\prime}\right) \in(y \downarrow f)_{0}$, the sets of objects and morphisms in the category $(y \downarrow f)\left((u, x),\left(u, x^{\prime}\right)\right)$ are defined by

$$
\begin{aligned}
(y \downarrow f)\left((u, x),\left(u^{\prime}, x^{\prime}\right)\right)_{0} & =\left\{(\theta, w) \in D_{2} \times C_{1} \mid \theta: u^{\prime} \Rightarrow u \circ f(w)\right\} \\
(y \downarrow f)\left((u, x),\left(u^{\prime}, x^{\prime}\right)\right)\left((\theta, w),\left(\theta^{\prime}, w^{\prime}\right)\right) & =\left\{\varphi \in C\left(x, x^{\prime}\right)\left(w, w^{\prime}\right) \mid \theta=(f(\varphi) \circ u) * \theta^{\prime}\right\} .
\end{aligned}
$$

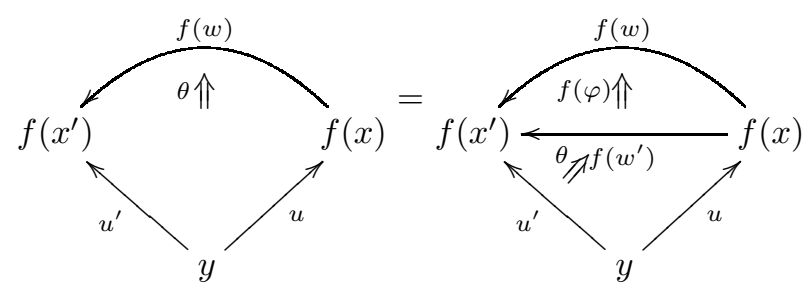


(c) For an object $(u, x) \in(y \downarrow f)_{0}$, define $1_{(u, x)}=\left(f_{x} \circ u, 1_{x}\right)$.

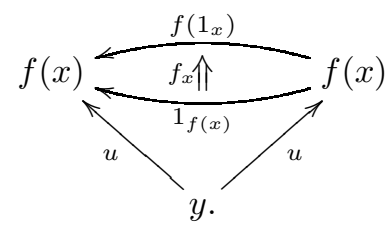

(d) The horizontal composition in $y \downarrow f$

$$
\circ:(y \downarrow f)\left(\left(u^{\prime}, x^{\prime}\right),\left(u^{\prime \prime}, x^{\prime \prime}\right)\right) \times(y \downarrow f)\left((u, x),\left(u^{\prime}, x^{\prime}\right)\right) \longrightarrow(y \downarrow f)\left((u, x),\left(u^{\prime \prime}, x^{\prime \prime}\right)\right)
$$

is defined by the following diagram

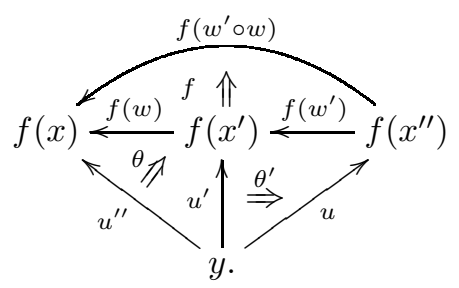

(e) The vertical composition in $y \downarrow f$ is given by that of $C$.

Remark A.43. For a colax functor $f, y \downarrow f$ cannot be defined, since the direction of $f_{x}: f\left(1_{x}\right) \Rightarrow$ $1_{f(x)}$ does not allow us to define an identity morphism on $(u, x)$. This is the only obstruction to defining $y \downarrow f$. When $f$ is normal, therefore, $y \downarrow f$ is defined. For example, a 1-morphism from $(u, x)$ to $\left(u^{\prime}, x^{\prime}\right)$ in $y \downarrow f$ is given by a pair $(\theta, w) \in D_{2} \times C_{1}$ with

$$
\theta: f(w) \circ u \Longrightarrow u^{\prime} .
$$

Horizontal compositions are given by the following diagram

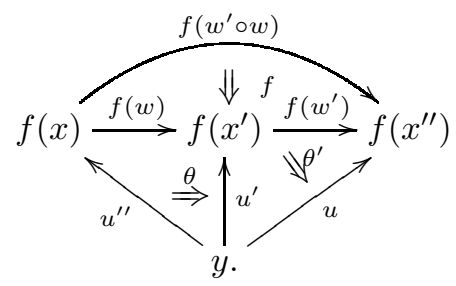

Similarly, for a normal lax functor, $f \downarrow y$ can be defined.

Lemma A.44. For a colax functor, the above data define a 2-category $f \downarrow y$. When $f$ is a lax functor, $y \downarrow f$ becomes a 2-category.

Proof. Consider the case of a colax functor. We need to verify the following:

1. $(f \downarrow y)\left((x, u),\left(x^{\prime}, u^{\prime}\right)\right)$ is a category for each pair $(x, u),\left(x^{\prime}, u^{\prime}\right) \in(f \downarrow y)_{0}$.

2. The horizontal composition (A.1) is a functor. 
3. The horizontal composition is associative.

4. $1_{(x, u)}$ serves as a unit for each $(x, u) \in(f \downarrow y)_{0}$.

Note that the set $(f \downarrow y)\left((x, u),\left(x^{\prime}, u^{\prime}\right)\right)_{1}$ is a subset of $C\left(x, x^{\prime}\right)_{1} \subset C_{2}$. It can be easily verified that $(f \downarrow y)\left((x, u),\left(x^{\prime}, u^{\prime}\right)\right)_{1}$ is closed under compositions. Thus $(f \downarrow y)\left((x, u),\left(x^{\prime}, u^{\prime}\right)\right)$ is a subcategory of $C\left(x, x^{\prime}\right)_{1}$.

The fact that (A.1) is a functor follows from the naturality of $f_{x^{\prime \prime}, x^{\prime}, x^{\prime}}: f\left(w^{\prime} \circ w\right) \Rightarrow f\left(w^{\prime}\right) \circ$ $f(w)$. The associativity for the horizontal composition follows from the commutativity of (3.1).

The fact that $1_{(x, u)}$ is a unit follows from the commutativity of (3.2) and (3.3).

Definition A.45. The 2-categories $f \downarrow y$ and $y \downarrow f$ are called the left homotopy fiber and the right homotopy fiber of $f$ over $y$, respectively.

When $C$ is a 1-category regarded as a 2-category, the homotopy fiber $f \downarrow y$ has a simpler description, since 2-morphisms in $f \downarrow y$ are defined by 2-morphisms in $C$.

Lemma A.46. Let $f: C \rightarrow D$ be a colax functor and suppose that $C$ is a 1-category. Then the left homotopy fiber $f \downarrow y$ is a 1-category for any object $y \in D_{0}$.

Corollary A.47. Let $f: C \rightarrow D$ be a colax functor from a poset $C$ to a poset-category $D$. Then the left homotopy fiber $f \downarrow y$ is a poset whose order relation $\leq_{y}$ is defined by

$$
(x, u) \leq_{y}\left(x^{\prime}, u^{\prime}\right) \Longleftrightarrow x \leq x^{\prime} \text { in } C \text { and } u^{\prime} \circ f\left(x \leq x^{\prime}\right) \leq u \text { in } D\left(f\left(x^{\prime}\right), y\right) \text {. }
$$

When $f$ is normal, the right homotopy fiber $y \downarrow f$ is also a poset whose order relation $\leq_{y}$ is defined by

$$
(u, x) \leq_{y}\left(u^{\prime}, x^{\prime}\right) \Longleftrightarrow x \leq x^{\prime} \text { in } C \text { and } f\left(x \leq x^{\prime}\right) \circ u \leq u^{\prime} \text { in } D\left(y, f\left(x^{\prime}\right)\right) .
$$

Proof. A morphism from $(x, u)$ to $\left(x^{\prime}, u^{\prime}\right)$ in $f \downarrow y$ is a pair $(\theta, w)$ with $\theta: u^{\prime} \circ f(w) \Rightarrow u$. Here $w$ is a morphism $w: x \rightarrow x^{\prime}$ in $C$ and $\theta$ is a morphism in $D(f(x), y)$. Since $C$ is a poset, such $w$ exists if and only if $x \leq x^{\prime} . D(f(x), y)$ is also a poset and thus $\theta$ exists if and only if $u^{\prime} \circ f\left(x \leq x^{\prime}\right) \leq u$. Furthermore such $w$ and $\theta$ are unique if exist. Thus $f \downarrow y$ is a poset.

When $f$ is normal, $y \downarrow f$ is defined. A morphism from $(u, x)$ to $\left(u^{\prime}, x^{\prime}\right)$ in $y \downarrow f$ is a pair $(\theta, w)$ with $\theta: f(w) \circ u \Rightarrow u^{\prime}$. Since both $C$ and $D\left(y, f\left(x^{\prime}\right)\right)$ are posets, such morphisms are unique and thus $y \downarrow f$ is a poset.

An analogue of $f^{-1}(x)$ can be defined as follows.

Definition A.48. For a colax functor $f: C \rightarrow D$ between 2-categories and an object $y \in D_{0}$, define a 2-subcategory $f^{-1}(y)$ of $C$ by

$$
\begin{aligned}
& f^{-1}(y)_{0}=\left\{x \in C_{0} \mid f(x)=y\right\} \\
& f^{-1}(y)_{1}=\left\{u \in C_{1} \mid f(u)=1_{y}\right\} \\
& f^{-1}(y)_{2}=\left\{\theta \in C_{2} \mid f(\theta)=1_{1_{y}}\right\} .
\end{aligned}
$$

Define

$$
j: f^{-1}(y) \longrightarrow f \downarrow y
$$

by $j(x)=\left(x, 1_{y}\right)$ on objects. For a 1-morphism $w: x \rightarrow x^{\prime}$ in $f^{-1}(y)$, define a 1-morphism in $f \downarrow y$ by the pair $\left(1_{1_{y}}, w\right) . j$ sends a 2 -morphism $\varphi$ in $f^{-1}(x)$ to $\varphi$. 
A generalization of Quillen's Theorem A to colax functors was proved by del Hoyo [dH12] based on the work [BC03] of Bullejos and Cegarra on Theorem A for 2-functors.

Theorem A.49 (Theorem A for colax functors). Let $f: C \rightarrow D$ be a colax functor between 2 -categories. If $B^{c l}(f \downarrow y)$ is contractible for every object $y \in D_{0}$, then the induced map

$$
B^{c l} f: B^{c l} C \longrightarrow B^{c l} D
$$

is a homotopy equivalence. When $f$ is normal, the induced map

$$
B^{n c l} f: B^{n c l} C \longrightarrow B^{n c l} D
$$

is a homotopy equivalence as long as $B^{\text {ncl }}(f \downarrow y)$ is contractible for every object $y \in D_{0}$.

We need the following version of a 2-categorical analogue of Corollary A.41. Recall from Lemma A.46 that homotopy fibers $f \downarrow y$ and $y \downarrow f$ are 1-categories, if the domain category $C$ of $f: C \rightarrow D$ is a 1-category. The fiber $f^{-1}(y)$ is also a 1-category.

Definition A.50. Let $f: C \rightarrow D$ be a colax functor from a small 1-category to a small 2category. We say $f$ is precofibered if the canonical inclusion $j_{y}: f^{-1}(y) \longrightarrow f \downarrow y$ has a left adjoint for each $y \in D_{0}$. Dually a lax functor $f: C \rightarrow D$ is said to be prefibered if the inclusion $i_{y}: f^{-1}(y) \rightarrow y \downarrow f$ has a right adjoint for each $y \in D_{0}$.

Corollary A.51. Let $f: C \rightarrow D$ be either a precofibered colax functor or a prefibered lax functor from a small 1-category $C$ to a small 2-category $D$. If $B f^{-1}(y)$ is contractible for each $y \in D_{0}$, $B f: B C \rightarrow B^{c l} D$ or $B f: B C \rightarrow B^{l} D$ is a homotopy equivalence. When $f$ is a prefibered normal colax functor or a precofibered normal lax functor, $B f: B C \rightarrow B^{n c l} D$ or $B f: B C \rightarrow B^{n l} D$ is a homotopy equivalence, respectively.

\section{References}

[BBL $\left.{ }^{+} 99\right]$ Eric Babson, Anders Björner, Svante Linusson, John Shareshian, and Volkmar Welker. Complexes of not $i$-connected graphs. Topology, 38(2):271-299, 1999, arXiv:math/9705219.

[BBM07] Mladen Bestvina, Kai-Uwe Bux, and Dan Margalit. Dimension of the Torelli group for $\operatorname{Out}\left(F_{n}\right)$. Invent. Math., 170(1):1-32, 2007, arXiv:math/0603177.

[BC03] M. Bullejos and A. M. Cegarra. On the geometry of 2-categories and their classifying spaces. K-Theory, 29(3):211-229, 2003, http://dx.doi.org/10.1023/B:KTHE.0000006921.50151.00.

[Bjö95] A. Björner. Topological methods. In Handbook of combinatorics, Vol. 1, 2, pages 1819-1872. Elsevier, Amsterdam, 1995.

[Bro60] Morton Brown. A proof of the generalized Schoenflies theorem. Bull. Amer. Math. Soc., 66:74-76, 1960.

[BW02] E. Batzies and V. Welker. Discrete Morse theory for cellular resolutions. J. Reine Angew. Math., 543:147-168, 2002, http://dx.doi.org/10.1515/crll.2002.012.

[CCG10] Pilar Carrasco, Antonio M. Cegarra, and Antonio R. Garzón. Nerves and classifying spaces for bicategories. Algebr. Geom. Topol., 10(1):219-274, 2010, arXiv:0903.5058. 
[Ceg11] A. M. Cegarra. Homotopy fiber sequences induced by 2-functors. J. Pure Appl. Algebra, 215(4):310-334, 2011, arXiv:0909.4229.

[CJS] R. L. Cohen, J.D.S. Jones, and G. B. Segal. Morse theory and classifying spaces, http://math. stanford.edu/ ralph/morse.ps. preprint.

[CJS94] R. L. Cohen, J. D. S. Jones, and G. B. Segal. Floer's infinite-dimensional Morse theory and homotopy theory. Sūrikaisekikenkyūsho Kōkyūroku, (883):68-96, 1994, http://www. kurims .kyoto-u.ac.jp/ kyodo/kokyuroku/contents/pdf/0883-4.pdf. Geometric aspects of infinite integrable systems (Japanese) (Kyoto, 1993).

[CJS95] R. L. Cohen, J. D. S. Jones, and G. B. Segal. Floer's infinite-dimensional Morse theory and homotopy theory. In The Floer memorial volume, volume 133 of Progr. Math., pages 297-325. Birkhäuser, Basel, 1995.

[Coh73] Marshall M. Cohen. A course in simple-homotopy theory. Springer-Verlag, New YorkBerlin, 1973. Graduate Texts in Mathematics, Vol. 10.

[Del08] Emanuele Delucchi. Shelling-type orderings of regular CW-complexes and acyclic matchings of the Salvetti complex. Int. Math. Res. Not. IMRN, (6):Art. ID rnm167, 39, 2008, arXiv:0705.3107.

[dH12] Matias L. del Hoyo. On the loop space of a 2-category. J. Pure Appl. Algebra, 216(1):28-40, 2012, arXiv:1005.1300.

[DS10] Emanuele Delucchi and Simona Settepanella. Combinatorial polar orderings and recursively orderable arrangements. Adv. in Appl. Math., 44(2):124-144, 2010, arXiv:0711.1517.

[Dug08] Danial Dugger. A primer on homotopy colimits, 2008, http://math.uoregon.edu/ ddugger/hocolim.pdf.

[Dus02] John W. Duskin. Simplicial matrices and the nerves of weak $n$-categories. I. Nerves of bicategories. Theory Appl. Categ., 9:198-308, 2001/02. CT2000 Conference (Como).

[For95] Robin Forman. A discrete Morse theory for cell complexes. In Geometry, topology, 83 physics, Conf. Proc. Lecture Notes Geom. Topology, IV, pages 112-125. Int. Press, Cambridge, MA, 1995.

[For98] Robin Forman. Morse theory for cell complexes. Adv. Math., 134(1):90-145, 1998, http://dx.doi.org/10.1006/aima.1997.1650.

[Fra79] John M. Franks. Morse-Smale flows and homotopy theory. Topology, 18(3):199-215, 1979, http://dx.doi.org/10.1016/0040-9383(79)90003-X.

[Gal10] Étienne Gallais. Combinatorial realization of the Thom-Smale complex via discrete Morse theory. Ann. Sc. Norm. Super. Pisa Cl. Sci. (5), 9(2):229-252, 2010, arXiv:0803.2616.

[Gra80] John W. Gray. Closed categories, lax limits and homotopy limits. J. Pure Appl. Algebra, 19:127-158, 1980, http://dx.doi.org/10.1016/0022-4049(80)90098-5.

[JW09] Michael Jöllenbeck and Volkmar Welker. Minimal resolutions via algebraic discrete Morse theory. Mem. Amer. Math. Soc., 197(923):vi+74, 2009. 
[Kal75] Gudrun Kalmbach. On some results in Morse theory. Canad. J. Math., 27:88-105, 1975 .

[Kas95] Christian Kassel. Quantum groups, volume 155 of Graduate Texts in Mathematics. Springer-Verlag, New York, 1995, http://dx.doi.org/10.1007/978-1-4612-0783-2.

[Koz05] Dmitry N. Kozlov. Discrete Morse theory for free chain complexes. C. R. Math. Acad. Sci. Paris, 340(12):867-872, 2005, arXiv:cs.DM/0504090.

[Koz08] Dmitry Kozlov. Combinatorial algebraic topology, volume 21 of Algorithms and Computation in Mathematics. Springer, Berlin, 2008, http://dx.doi.org/10.1007/978-3-540-71962-5.

[Lac10] Stephen Lack. A 2-categories companion. In Towards higher categories, volume 152 of IMA Vol. Math. Appl., pages 105-191. Springer, New York, 2010, arXiv:math/0702535.

[LW69] Albert T. Lundell and Stephen Weingram. Topology of CW-Complexes. Van Nostrand Reinhold, New York, 1969.

[May72] J. P. May. The geometry of iterated loop spaces. Springer-Verlag, Berlin, 1972.

[ML98] Saunders Mac Lane. Categories for the working mathematician, volume 5 of Graduate Texts in Mathematics. Springer-Verlag, New York, 1998.

[MN13] Konstantin Mischaikow and Vidit Nanda. Morse Theory for Filtrations and Efficient Computation of Persistent Homology. Discrete Comput. Geom., 50(2):330-353, 2013, http://dx.doi.org/10.1007/s00454-013-9529-6.

[Qui73] Daniel Quillen. Higher algebraic K-theory. I. In Algebraic K-theory, I: Higher Ktheories (Proc. Conf., Battelle Memorial Inst., Seattle, Wash., 1972), pages 85-147. Lecture Notes in Math., Vol. 341. Springer, Berlin, 1973.

[RS71] C. P. Rourke and B. J. Sanderson. $\triangle$-sets. I. Homotopy theory. Quart. J. Math. Oxford Ser. (2), 22:321-338, 1971.

[Rus73] T. Benny Rushing. Topological embeddings. Academic Press, New York-London, 1973. Pure and Applied Mathematics, Vol. 52.

[Seg68] Graeme Segal. Classifying spaces and spectral sequences. Inst. Hautes Études Sci. Publ. Math., 34:105-112, 1968.

[Seg73] Graeme Segal. Configuration-spaces and iterated loop-spaces. Invent. Math., 21:213221, 1973.

[Seg74] Graeme Segal. Categories and cohomology theories. Topology, 13:293-312, 1974.

[Sha01] John Shareshian. Discrete Morse theory for complexes of 2-connected graphs. Topology, 40(4):681-701, 2001, http://dx.doi.org/10.1016/S0040-9383(99)00076-2.

[Skö06] Emil Sköldberg. Morse theory from an algebraic viewpoint. Trans. Amer. Math. Soc., 358(1):115-129 (electronic), 2006, http://dx.doi.org/10.1090/S0002-9947-05-04079-1. 
[SS07] Mario Salvetti and Simona Settepanella. Combinatorial Morse theory and minimality of hyperplane arrangements. Geom. Topol., 11:1733-1766, 2007, arXiv:0705.2874.

[Str96] Ross Street. Categorical structures. In Handbook of algebra, Vol. 1, volume 1 of Handb. Algebr., pages 529-577. Elsevier/North-Holland, Amsterdam, 1996, http://dx.doi.org/10.1016/S1570-7954(96)80019-2.

[Wat66] Charles E. Watts. A homology theory for small categories. In Proc. Conf. Categorical Algebra (La Jolla, Calif., 1965), pages 331-335. Springer, New York, 1966. 